\title{
LYON NATURAL RADIOCARBON MEASUREMENTS IX
}

\section{JACQUES EVIN, JOELLE MARECHAL, and GERARD MARIEN}

Laboratoire de radiocarbone, Centre de Datation et d'Analyses isotopiques, Université Claude-Bernard de Lyon

43 boulevard du 11 Novembre 1918, 69621 Villeurbanne, France

\section{INTRODUCTION}

This list includes most of the measurements made in 1979 through 1981 and some values obtained during preceding years. The reporting of results, their calculation (half-life: $5570 \pm 0$, standard ${ }^{13} \mathrm{C}$ correction only for bones), and the dilution ratios are as previously described in Lyon VIII (R, 1979, v 21, p 402-452).

The counting technique using proportional detectors has now been completely abandoned; only a few measurements on water samples and the Chassey series were measured in this way. Almost all analyses were made using the three Packard liquid scintillation spectrometers which were specially shielded with $5 \mathrm{~cm}$ of lead and placed in an underground room beneath 3 to $4 \mathrm{~m}$ of earth. Ca 30 glass counting vessels were selected. The differences in their backgrounds did not exceed $0.2 \mathrm{cpm}$. The backgrounds of the 3 routine counters were, respectively: $1.9 \pm 0.1,2.2 \pm 0.1$, and $3.2 \pm 0.2 \mathrm{cpm}$. Adjustments to yields of ca $55 \%$ were made by the quenching correction of simultaneous counting on two channels corresponding to different window opening. For these conditions, $3 \mathrm{ml}$ of benzene from the oxalic acid standard gave an uncorrected counting rate of ca $17 \mathrm{cpm}$.

Pretreatment of samples was according to the type of dating material, conforming to generally applied methods and particular archaeologic or geologic problems. We took into account some experiments made on specific dating materials (paleosoil, terrestial shells) ( $\mathrm{R}, 1980, \mathrm{v} 22$, p 545-555, 919-929) which confirm previous studies made by other laboratories, the synthesis of which will soon be published (Evin, 1983). These studies demonstrated the often inadequate elimination of contamination by basic treatment or hydrolysis on organic material or by superficially acid washing of carbonaceous material. Pretreatment of bones, which seems satisfactory, remains the same and is uniformly applied, always following the procedure of Longin (1971).

\section{ACKNOWLEDGMENTS}

We thank Gérard Drevon who contributed to the routine preparation of most of the samples. We are indebted to Claude-Bernard University for administrative and partial financial support and are grateful to the staff of the Nuclear Physics Institute for technical assistance. 


\section{SAMPLE DESCRIPTIONS}

\section{EXPERIMENTAL SAMPLES}

The following samples were measured often on laboratory request 1 ) to check laboratory adjustment with other laboratories (Ly-2224 and Chêne de Stolford series, 2) to verify reliability of some dating materials (Paléosol de Beylongue series, Ly-1884 and -2500), 3) to demonstrate utility and accuracy of ${ }^{14} \mathrm{C}$ measurements for commercial (Ly-2223 and aromatic components series) or artistic (Ly-1854 and -1907) purposes.

\section{Ly-2224. Bois de Berne}

$2330 \pm 90$

Wood from unknown origin subm 1980 by $\mathrm{T}$ Riesen, Radiocarbon Lab, Bern, Switzerland (5427 min count). Comment: agrees perfectly with Bern measurement: B-3499, $2220 \pm 100$.

\section{Chêne de Stolford series, Wales}

Wood from submerged forest coll 1979 by A Heyworth, Univ Aberystwyth and subm 1980 by M S Baxter, Univ Glasgow in participation with Lyon lab Internatl Tree-Ring Replicate Study. Results appear in table 1. Sampling of wood, selection of proposed material, and preparation procedures were previously pub with synthesis of results (Baxter, 1983).

TABLE 1

Chêne de Stolford

\begin{tabular}{clccc}
\hline Sample no. & \multicolumn{1}{c}{$\begin{array}{c}\text { Wood } \\
\text { ref }\end{array}$} & $\begin{array}{c}\text { Counting } \\
\text { time }\end{array}$ & $\begin{array}{c}\text { Dilution } \\
\text { ratio }\end{array}$ & $\begin{array}{c}\text { Conventional } \\
\text { age }\end{array}$ \\
\hline Ly-2157 & Stolford $147-156$ & $6872 \mathrm{~min}$ & $2 / 5$ & $5070 \pm 90$ \\
-2225 & Stolford $118-127$ & $3339 \mathrm{~min}$ & 1 & $5120 \pm 130$ \\
-2156 & Stolford $88-89$ & $10,161 \mathrm{~min}$ & 1 & $5175 \pm 60$ \\
-2226 & Stolford $60-69$ & $3878 \mathrm{~min}$ & $14 / 15$ & $5200 \pm 160$ \\
-2155 & Stolford $31-40$ & $6503 \mathrm{~min}$ & $2 / 5$ & $5330 \pm 100$ \\
\hline
\end{tabular}

General Comment: results agree in general with those obtained by 20 other labs. Comparison of counting times between different statistical margins shows that errors necessarily made during successive phases of preparation and counting of each sample (eg, errors in weighing sample, counting standard, background stability of purity corrections, etc) become preponderant with respect to statistics for counting times longer than 5000 minutes. Taking into account only counting statistics would lead to underestimate of error.

\section{Paléosol de Beylongue series, Morcenx, Landes}

Peaty sediments from Layer 5 in Beylongue geol sec $\left(44^{\circ} 00^{\prime} \mathrm{N}, 0^{\circ} 55^{\prime}\right.$ W). Coll and subm 1971 by $C$ Thibault and P Legigan, Univ Bordeaux. Layer is interstadial or interglacial paleosoil attributed to Würmian period. Measurement was made to strengthen conclusions of study on evolution of organic matter in soil and sample treatment methods of paleosoil datings (Gilet-Blain, Marien, and Evin, 1980). 
Ly-1537. Hydrolysat

$13,960 \pm 360$

Soluble fraction of acid hydrolyse. (2/3 diluted sample)

Ly-1538. Reliquat

$16,760 \pm 440$

Insoluble fraction after several acid hydrolyse. (9/10 diluted sample)

General Comment: as suggested by general study, age is probably too young since last interstadial (long enough to form a paleosoil) occurred $17,000 \mathrm{yr}$ ago. Both results indicate increasing age according to chemical evolution conditions of soil organic matter and only give min age for sediments (Evin et al, 1979).

\section{Ly-1884. Trébous II D I, Deyme, Haute Garonne $\quad 29,200$}

Shells and opercula of gastropods from silt $\left(43^{\circ} 29^{\prime} \mathrm{N}, 1^{\circ} 31^{\prime} \mathrm{E}\right)$. Coll 1979 by J C Revel, Lab Pédol, Univ Toulouse. Sample from bottom of geol series of sediments, organic matter of which were dated by Monaco Lab (unpub) of ca 23,000 $\pm 1000 \mathrm{BP}$. Comment (JCR): date agrees with expected range of dates and indicates Late Würmian age but disagrees with results from organic matter. However, both values may be considered min ages. True age may be older and observed ${ }^{14} \mathrm{C}$ activities may come from contamination which can never be eliminated from such dating materials (Evin et al, 1980; Gilet-Blain, Marien, and Evin, 1980).

\section{Ly-2500. Tombe 142, Casabianda, Aléria, Haute-Corse}

$2890 \pm 120$

Hélix shells from inner wall of Grave 142 of Casabianda necropolis $\left(42^{\circ} 05^{\prime} \mathrm{N}, 9^{\circ} 30^{\prime} \mathrm{E}\right)$. Coll 1971 by $\mathrm{J}$ and $\mathrm{L}$ Jehasse, preserved in Jérôme Carcopino Mus Aléria, and subm 1981 by J Evin. Graves were dug in sandy clays and precisely dated by assoc archaeol material from Greece at beginning of 5th century BC; snails are from this last period or a little younger. Comment: date is ca $500 \mathrm{yr}$ too old and confirms previous findings (Evin et al, 1980) on impossibility of using shells of terrestrial gastropods for dating archaeol sites.

Ly-2223. Gypsum mine, Carcès, Var

$2570 \pm 130$

Wood found in gypsum mine $\left(43^{\circ} 29^{\prime} \mathrm{N}, 6^{\circ} 11^{\prime} \mathrm{E}\right)$. Coll and subm 1980 by G Truc, Dept Geol, Univ Lyon. Comment (GT): recent age of wood indicates it came from ancient timbers and embedding sediments are collapse of ancient gallery.

\section{Aromatic components series}

Aromatic organic components are present in such products as perfume, aperitifs, alimentary adjuvants, etc, from various origins (synthetic or natural, homogeneous or mixed). Subm from 1978 to 1980 by industria? firms or fraud-control labs. All samples were very slowly burned in pure oxygen after placement on quartz wool (table 2). 
TABLE 2

Aromatic components

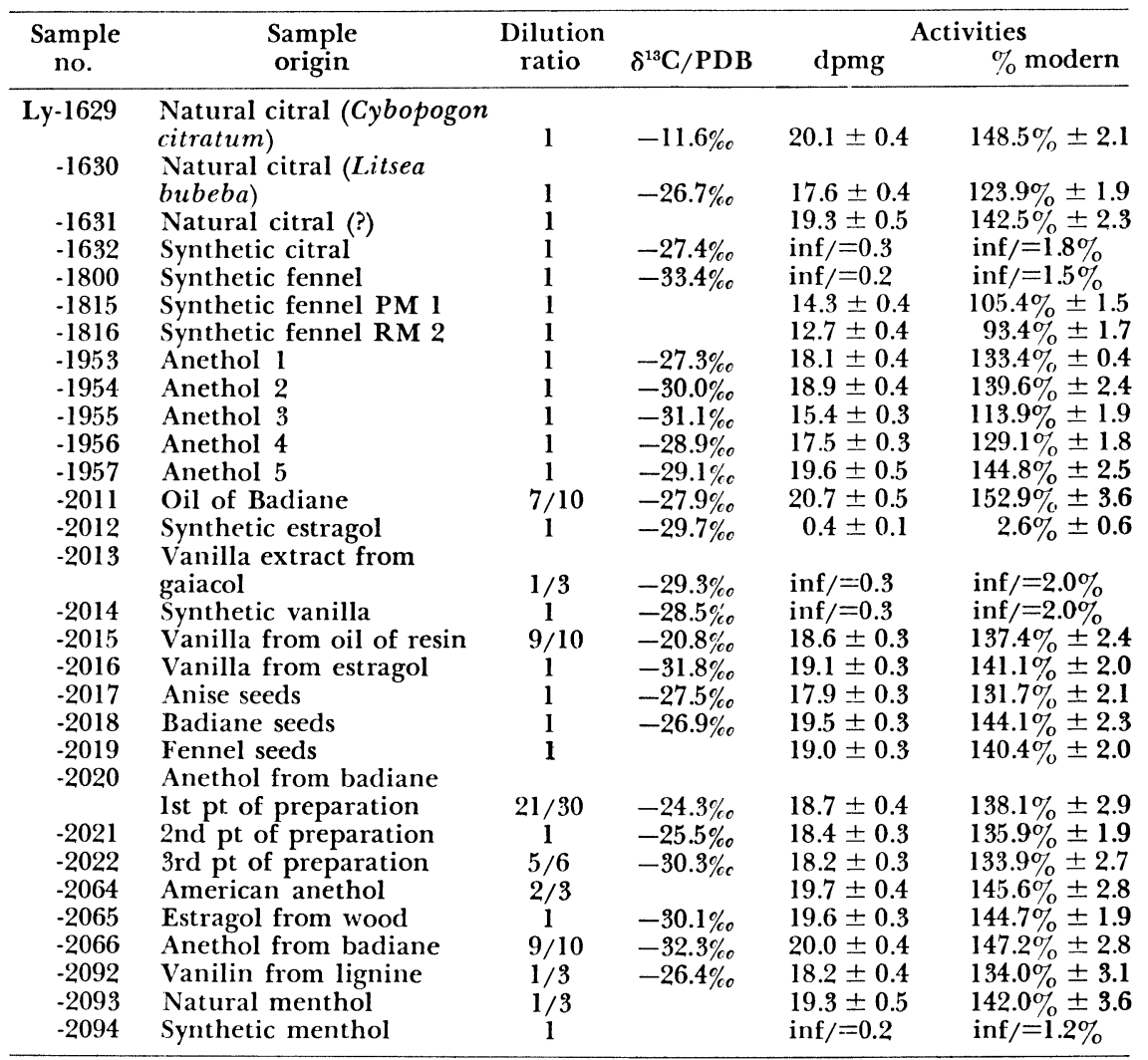

General Comment: differences among results indicate need for determining sample origins. Some samples have activities lower than $16 \mathrm{dpmg}$ which must represent mixing of natural components with synthetic chemical products. Some amounts may be measured but extracts are not distinguishable from seeds and woods. Three values for Ly-2020, -2021, and -2023 from several phases of same preparation indicate slight isotopic fractionation during operation of an industrial lab. Such differences are small but may be consistent enough to explain differences among products extracted from natural components grown in same year (Bricout \& Koziet, 1978).

Ly-1854. Statuette de Crémieu

$260 \pm 150$

Wood from pedestal of statuette attributed by style to 11th or 12th century. Coll 1979 in Antiquities Market at Crémieu, Isère $\left(45^{\circ} 53^{\prime} \mathrm{N}\right.$, $\left.5^{\circ} 15^{\prime} \mathrm{E}\right)$, from unknown origin and subm 1979 by $\mathrm{G}$ Villedieu, Villeurbanne. Measurement made to authenticate artifact. Comment (GV): date much younger than expected and suggests statuette was sculpted in Middle-Age style from old wood. 
Ly-1907. Statuette chinoise de Tch'ang-cha, Hou-Nan, China

$2370 \pm 140$

Wood from Chinese statuette attributed to Han epoch (206-220 вC). Coll at Tch'ang-cha $\left(28^{\circ} 05^{\prime} \mathrm{N}, 113^{\circ} 01^{\prime} \mathrm{E}\right)$. Subm 1979 by C T Loo, Paris. As such statuettes often were imitated, measurement was made to authenticate artifact. Comment (CTL): date agrees with expected old age and indicates beginning of Han period, but only gives age of wood on which statuette was sculpted.

11. GEOLOGIC SAMPLES

A. Samples from peat bogs

Ly-1940. Marais de Chautagne, Serrière-enChautagne, Savoie

$1170 \pm 140$

Peat from top of peaty layer several $\mathrm{m}$ thick in Chautagne peat bog $\left(45^{\circ} 53^{\prime} \mathrm{N}, 5^{\circ} 50^{\prime} \mathrm{E}\right)$. Coll 1978 by Co Natle Rhône and subm 1979 by $\mathrm{M}$ Bornand, Inst Recherche Agronom, Montpellier. Layer overlies thick fluvio-glacial gravels. Dated to determine min age of gravels which blocked Le Bourget Lake outlet. Comment (MB): dates end of peat accumulation, and not formation of lake dam (Bornand \& Guyon, 1979).

Ly-2298. Le Lit-au-Roi, Cressin, Ain

$1900 \pm 120$

Peat from base of peaty layer $1.5 \mathrm{~m}$ thick in peat bog $\left(45^{\circ} 35^{\prime} \mathrm{N}, 5^{\circ}\right.$ $43^{\prime}$ E). Coll and subm 1980 by R Vilain, Dept Geol, Univ Lyon. Peat bog formed at final stage of filling of glacial lake. Comment (RV): date, much younger than expected, indicates rapidity of peat formation and recent filling of lake.

Ly-2349. Saint-Paul-les-Durance, Bouches du Rhône $6870 \pm 160$

Peaty slime from 10.6 to $10.9 \mathrm{~m}$ depth in boring at point PK 4800 near bank of Durance Canal $\left(49^{\circ} 29^{\prime} \mathrm{N}, 5^{\circ} 42^{\prime} \mathrm{E}\right)$. Coll 1967 and subm 1980 by J L de Beaulieu, Lab Bot Hist Palynol, Univ Marseille. Level was attributed to Riss-Würm interglacial period elsewhere called "Eemian" (Beaulieu, 1972; Bonifay, 1962). Comment (JL de B): date is much younger than expected, dating peaty formation to Holocene; $c f$ another measurement on calcareous tufa lying close to peaty level, MC-2171: 7000 \pm 100 (Farizier, 1980). Discrepancy between results and previous geol or palynol "Eemian" attribution is now under study.

\section{Lago Zapano series, Lagonegro, Campania, Italy}

Organic clay from three levels in basal sediments of Zapano Lake $\left(40^{\circ} 09^{\prime} \mathrm{N}, 15^{\circ} 50^{\prime} \mathrm{E}\right)$. Coll and subm 1978 by M Reille, Lab Palynol, Univ Marseille. Pollen diagram indicates very recent age (end of SubAtlantic period) while clay facies suggest interglacial origin.

Ly-2253. II, 119-121 cm

From bottom of a peaty layer in clays; presence of Fagus and cereal pollen. (11/30 diluted sample) 
Ly-2254. II, 124-126cm

$700 \pm 170$

From top layer of clay; large amounts of Fagus and Ablies. (1/2 diluted sample)

\section{Ly-2255. III, 143-147 cm}

$1290 \pm 170$

From mid-layer of clay; presence of leaf remains. (1/2 diluted sample) General Comment (MR): as expected, peaty layer (Ly-2253) is very recent. Both other dates confirm absence of lacuna between organic clay and peaty layer and that, despite interglacial facies, clay is also recent.

\section{Ly-1774. Lago Laceno, Bagnoli d'Irpino, Campania, Italy}

Organic clay from 380 to $387 \mathrm{~cm}$ depth in lake sediments $\left(40^{\circ} 45^{\prime} \mathrm{N}\right.$, $15^{\circ} 7^{\prime} \mathrm{E}$ ). Coll 1977 and subm 1977 by $M$ Reille. (5/6 diluted sample). Comment (MR): presence of Fagus and depth suggest either interglacial level ( $>30,000 \mathrm{BP}$ ) or rapid sedimentation rate. Date confirms latter.

\section{Ly-2319. Bourricos, Pontenx les Forges, Landes $2070 \pm 130$}

Peat from $30 \mathrm{~cm}$ depth in compact peaty layer $80 \mathrm{~cm}$ thick, outcropping in quarry $\left(44^{\circ} 09^{\prime} \mathrm{N}, 0^{\circ} 65^{\prime} \mathrm{W}\right)$. Coll 1980 and subm 1981 by $\mathrm{P}$ Legigan, Univ Bordeaux. Comment (PL): palynol confirms entire layer formed between Sub-boreal and present.

\section{Pré Maudit series, Gathemo, Manche}

Peat or clay with organic matter from three levels of core $\left(48^{\circ} 45^{\prime} \mathrm{N}\right.$, $0^{\circ} 97^{\prime} \mathrm{W}$ ). Coll and subm 1981 by L Barthélemy, Centre Géog Phys, Univ Paris-X, Nanterre.

\section{Ly-2407. 308}

$6870 \pm 170$

Peat from 331 to $334 \mathrm{~cm}$ depth. Pollen indicates humidification phase with Betula, Alnus, and Quercetum mixtum, attributed to Atlantic period. (5/6 diluted sample)

\section{Ly-2405. $\quad 307$}

$7450 \pm 180$

Peat from ca $340 \mathrm{~cm}$ depth. Decrease in pollen of Corylus, increase of Betula, Quercetum mixtum, Alnus, Myrica gale, Salix, attributed to beginning of Atlantic. (1/2 diluted sample)

Ly-2406. 305

$9250 \pm 180$

Clay with organic matter from 353 to $357 \mathrm{~cm}$ depth. Dominance of Corylus, assoc with Alnus, Betula, and increase of total arboreal pollen attributable to beginning of Atlantic period. (3/5 diluted sample)

General Comment (LB): Ly-2407 and -2405 confirm geomorphol and palynol conclusions. Ly-2406 corresponds to Pre-boreal period and suggests peat bog settled during Alleröd with sedimentation hiatus occurring during Boreal. 


\section{Seillons-Source d'Argens series, Var}

Peat from three levels of peat bog $2.5 \mathrm{~km} \mathrm{E}$ of Seillons $\left(43^{\circ} 30^{\prime} \mathrm{N}, 5^{\circ}\right.$ $52^{\prime}$ E). Coll 1979 and subm 1980 by H Triat-Laval, Lab Palynol, Univ Marseille (Triat-Laval, 1981).

Ly-2218. 110-120cm

$2200 \pm 100$

Pollen diagram indicates increase in deforestation which had begun previously; Sub-atlantic age expected.

Ly-2119. 190-200cm

$4080 \pm 130$

Pollen diagram indicates small clearing of wood at this level; Subboreal age expected.

\section{Ly-2220. $330-340 \mathrm{~cm}$}

$4650 \pm 110$

From base of peat bog at level where pollen diagram indicates forest of decicluous trees existed before start of deforestation: Atlantic age expected.

General Comment: three dates agree perfectly with expected range of dates. Only $600 \mathrm{yr}$ and $1.3 \mathrm{~m}$ sediment between Ly-2220 and -2219 indicates rapid sedimentation for beginning of filling.

Ly-1583. Praveille, La Versanne, Loire

$7410 \pm 190$

Peat from 1.2 to $1.3 \mathrm{~m}$ depth at bottom of acid, raised peat bog on side of Pilat massif, near Le Grand-Bois pass $\left(45^{\circ} 19^{\prime} \mathrm{N}, 4^{\circ} 30^{\prime} \mathrm{E}\right)$. Coll and subm 1977 by N Gilet and A M Domenach, Lab Ecol Végétale, Univ Lyon. Pollen diagram by $\mathrm{H}$ Méon shows extension of Tilia and presence of Quercus and Alnus, ie, beginning of relatively warm climate. Comment (AMD): date agrees with palynol as it shows beginning of Atlantic period when slight increase of temperature occurred.

\section{Casabianda series, Aléria, Haute Corse}

Peat from top and basal layers of a peaty horizon embedded in slimy basal sediments of pond $\left(42^{\circ} 15^{\prime} \mathrm{N}, 7^{\circ} 10^{\prime} \mathrm{E}\right)$. Coll and subm 1980 by $\mathrm{M}$ Reille.

Ly-2257. Top

$5650 \pm 190$

From top of peaty layer just underlying layer in which Quercus ilex, Olea sp, and cereal pollen indicate Sub-atlantic period. (1/2 diluted sample)

Ly.2256. Base

From base of peaty layer just overlying layer in which pollen clearly indicates Atlantic period. ( $3 / 5$ diluted sample)

General Comment (MR): both dates confirm that peaty layer belongs to Atlantic period and sedimentation hiatus occurred on top of peat.

\section{Bordure Nord du Massif du Cantal series, Cantal}

Seven borings were made in four peat bogs (table 3) at ca $1200 \mathrm{~m}$ alt in Cantal massif, and table 4 lists results of samples of peat and organic clay coll and subm by M Reille and J L de Beaulieu, Lab Palynol, Univ Marseille. 
TABLE 3

Bordure Nord du Massif Cantal—sampling sites

\begin{tabular}{lllll}
\hline Boring & \multicolumn{1}{c}{ Village } & Loc & Colln yr & Subm yr \\
\hline Le Joland I & Ségur les Villas & $\left(45^{\circ} 12^{\prime} \mathrm{N}, 2^{\circ} 50^{\prime} \mathrm{E}\right)$ & 1979 & 1980 \\
Le Joland II & Ségur les Villas & $\left(45^{\circ} 12^{\prime} \mathrm{N}, 2^{\circ} 50^{\prime} \mathrm{E}\right)$ & 1981 & 1981 \\
Brugeroux & Chastel sur Murat & $\left(45^{\circ} 09^{\prime} \mathrm{N}, 2^{\circ} 50^{\prime} \mathrm{E}\right)$ & 1978 & 1978 \\
La Taphanel III & Riom es Montagne & $\left(45^{\circ} 16^{\prime} \mathrm{N}, 2^{\circ} 41^{\prime} \mathrm{E}\right)$ & 1978 & 1980 \\
La Taphanel IV & Riom es Montagne & $\left(45^{\circ} 16^{\prime} \mathrm{N}, 2^{\circ} 41^{\prime} \mathrm{E}\right)$ & 1978 & 1981 \\
La Taphanel II & Riom es Montagne & $\left(45^{\circ} 16^{\prime} \mathrm{N}, 2^{\circ} 41^{\prime} \mathrm{E}\right)$ & 1978 & 1982 \\
Lastioules & Champs sur Tarentaise & $\left(45^{\circ} 29^{\prime} \mathrm{N}, 2^{\circ} 39^{\prime} \mathrm{E}\right)$ & 1979 & 1980 \\
\hline
\end{tabular}

TABLE 4

Bordure Nord du Massif Cantal—samples

\begin{tabular}{|c|c|c|c|c|c|}
\hline $\begin{array}{l}\text { Sample } \\
\text { no. }\end{array}$ & Boring & Depth & $\begin{array}{c}\text { Expected } \\
\text { climatic phase }\end{array}$ & $\begin{array}{l}\text { Dilution } \\
\text { ratio }\end{array}$ & Age BP \\
\hline Ly-2494 & Le Joland II & $50-60 \mathrm{~cm}$ & Post-Middle age & $1 / 15$ & $1280 \pm 360$ \\
\hline-2495 & Le Joland II & $90-100 \mathrm{~cm}$ & Middle age & $3 / 10$ & $1030 \pm 160$ \\
\hline-2132 & Le Joland I & $230-240 \mathrm{~cm}$ & Sub-atlantic & $1 / 4$ & $2850 \pm 260$ \\
\hline-2496 & Le Joland II & $150-160 \mathrm{~cm}$ & End of Sub-boreal & $1 / 2$ & $2610 \pm 150$ \\
\hline-2133 & Le Joland I & $300-310 \mathrm{~cm}$ & Sub-boreal & $1 / 6$ & $2670 \pm 320$ \\
\hline-2497 & Le Joland II & $250-260 \mathrm{~cm}$ & Sub-boreal & $11 / 30$ & $4410 \pm 210$ \\
\hline-2498 & Le Joland II & $290-297 \mathrm{~cm}$ & End of Atlantic & $1 / 3$ & $5880 \pm 200$ \\
\hline-2134 & Le Joland I & $440-450 \mathrm{~cm}$ & End of Atlantic & $2 / 3$ & $5350 \pm 210$ \\
\hline-2447 & Lastioules & $40-45 \mathrm{~cm}$ & End of Sub-boreal & $1 / 6$ & $2460 \pm 210$ \\
\hline-2448 & Lastioules & $128-135 \mathrm{~cm}$ & $\begin{array}{l}\text { Beginning of Sub- } \\
\text { boreal }\end{array}$ & $1 / 5$ & - \\
\hline-2555 & La Taphanel II & $355-370 \mathrm{~cm}$ & Sub-boreal & $3 / 10$ & $2800 \pm 160$ \\
\hline-2135 & La Taphanel III & $45-55 \mathrm{~cm}$ & Sub-atlantic & $3 / 5$ & $2890 \pm 180$ \\
\hline-2136 & La Taphanel III & $105-110 \mathrm{~cm}$ & Sub-boreal & $1 / 2$ & $4130 \pm 190$ \\
\hline-2137 & La Taphanel III & $135-140 \mathrm{~cm}$ & $\begin{array}{l}\text { Beginning of Sub- } \\
\text { boreal }\end{array}$ & 1 & \\
\hline-2138 & La Taphanel III & $200-205 \mathrm{~cm}$ & Middle Boreal & 1 & \pm 150 \\
\hline-2139 & La Taphanel III & $255-260 \mathrm{~cm}$ & Beginning of Atlat & 1 & $7520 \pm 150$ \\
\hline-2140 & La Taphanel III & $335-340 \mathrm{~cm}$ & Middle Atlantic & 1 & $8440 \pm 160$ \\
\hline-2141 & La Taphanel III & $405-410 \mathrm{~cm}$ & Beginning of Boreal & $1 / 2$ & $9700 \pm 230$ \\
\hline-2142 & La Taphanel III & $415-420 \mathrm{~cm}$ & End of Pre-boreal & $9 / 30$ & $9530 \pm 200$ \\
\hline-2143 & La Taphanel III & $435-440 \mathrm{~cm}$ & $\begin{array}{l}\text { Beginning of Pre- } \\
\text { boreal }\end{array}$ & $2 / 5$ & \\
\hline-2144 & La Taphanel III & $445-455 \mathrm{~cm}$ & End of Late Dryas & $7 / 15$ & $10,390 \pm 230$ \\
\hline-2145 & La Taphanel III & $460-470 \mathrm{~cm}$ & Late Dryas & $1 / 3$ & $10,450 \pm 250$ \\
\hline-2212 & La Taphanel IV & $565-576 \mathrm{~cm}$ & Early Dryas & $3 / 5$ & $12,380 \pm 210$ \\
\hline-2361 & La Taphanel IV & $610-630 \mathrm{~cm}$ & Early Dryas & $7 / 30$ & $10,780 \pm 410$ \\
\hline-2119 & Brugeroux & $350-360 \mathrm{~cm}$ & Boreal & $1 / 4$ & $8310 \pm 300$ \\
\hline-2120 & Brugeroux & $450-500 \mathrm{~cm}$ & Pre-boreal & $3 / 8$ & $9860 \pm 280$ \\
\hline-1855 & Brugeroux & $556-564 \mathrm{~cm}$ & $\begin{array}{l}\text { Beginning of Pre- } \\
\text { boreal }\end{array}$ & $7 / 15$ & - \\
\hline-1856 & Brugeroux & $575-585 \mathrm{~cm}$ & Late Dryas & $7 / 15$ & $10,270 \pm 430$ \\
\hline-2121 & Brugeroux & $588-600 \mathrm{~cm}$ & Alleröd & $7 / 15$ & $10,790 \pm 240$ \\
\hline-2122 & Brugeroux & $630-640 \mathrm{~cm}$ & Beginning of Alleröd & $1 / 2$ & $11,450 \pm 240$ \\
\hline-2123 & Brugeroux & $645-655 \mathrm{~cm}$ & Bölling & $4 / 15$ & $12,350 \pm 360$ \\
\hline-2124 & Brugeroux & $660-670 \mathrm{~cm}$ & Early Dryas & $1 / 20$ & $11,610 \pm 850$ \\
\hline
\end{tabular}

General Comment (MR \& JL, de B): Holocene results agree with values expected from pollen study. Late-glacial results are less accurate because of low carbon content of samples; these seem consistent, especially La Taphanel series from 435 to $470 \mathrm{~cm}$ where they well define Late Dryas period. Ly-2122 and -2361 are too young for unknown reasons. 


\section{Le Cézalier Massif series, Puy de Dôme}

Table 5 lists results obtained from clay with little organic content from several levels in cores from three neighboring sites at $1300 \mathrm{~m}$ alt: Les Chastelets and Le Lac d'En Bas boring near La Godivelle $\left(45^{\circ} 23^{\prime} \mathrm{N}, 2^{\circ}\right.$ $\left.55^{\prime} \mathrm{E}\right)$, and Jassy boring near Saint-Alyre-es-Montagne, $\left(45^{\circ} 23^{\prime} \mathrm{N}, 2^{\circ} 58^{\prime}\right.$ E). Coll and subm 1979 by M Reille.

TABLE 5

Massif du Cézalier

\begin{tabular}{|c|c|c|c|c|c|}
\hline $\begin{array}{c}\text { Sample } \\
\text { no. }\end{array}$ & Boring & Depth & $\begin{array}{l}\text { Expected } \\
\text { climatic } \\
\text { phase }\end{array}$ & $\begin{array}{l}\text { Dilution } \\
\text { ratio }\end{array}$ & Age BP \\
\hline Ly-2260 & Les Chastelets & $120-130 \mathrm{~cm}$ & Sub-atlantic & $2 / 15$ & $2940 \pm 210$ \\
\hline-2261 & Les Chastelets & $190-200 \mathrm{~cm}$ & Sub-atlantic & $4 / 15$ & $2600 \pm 230$ \\
\hline-2262 & Les Chastelets & $240-250 \mathrm{~cm}$ & Sub-atlantic & $7 / 30$ & $3020 \pm 240$ \\
\hline-2263 & Les Chastelets & $850-860 \mathrm{~cm}$ & Atlantic & $1 / 15$ & $19,400 \pm 1560$ \\
\hline-21$] 7$ & Le Lac d'En Bas & $680-690 \mathrm{~cm}$ & Atlantic & $1 / 9$ & $5590 \pm 410$ \\
\hline$-21 \| 8$ & Le Lac d'En Bas & $800-810 \mathrm{~cm}$ & Atlantic & $2 / 13$ & $6070 \pm 320$ \\
\hline-2446 & Jassy & $240-245 \mathrm{~cm}$ & Atlantic & $1 / 6$ & $5040 \pm 330$ \\
\hline
\end{tabular}

General Comment (MR): 1st 3 results of Les Chastelets series fit in expected range of dates despite large uncertainty and stratigraphic inversion. Ly-2261 is too young. Ly-2263 is aberrant and remains unexplained. Both results from Le Lac d'En Bas seem too old by ca $1000 \mathrm{yr}$ compared with palynol data in region and Ly-2446 which seems to be most reliable of series. Small amounts of available carbon may cause discrepancies between results and expected values despite lengthening of counting times.

\section{Massif de la Margeride series, Lozère et Haute-Loire}

Peat from several borings at ca $1300 \mathrm{~m}$ alt. Coll and subm 1980 by A Pons and M Reille.

\section{Ly-2360. Mont-Chauvet I, Malzieu-Forain, Modern Lozère \\ $\Delta^{14} \mathrm{C}=-1.6 \pm 1.2 \%$}

From 130 to $140 \mathrm{~cm}$ depth $\left(44^{\circ} 55^{\prime} \mathrm{N}, 1^{\circ} 15^{\prime} \mathrm{E}\right)(3 / 10$ diluted sample). Pollen diagram indicates beginning of last extension of Pinus and cereals, ie very recent (ca 200 to $300 \mathrm{BP}$ ).

Ly-2356. Sainte-Eulalie, Lozère $2120 \pm 170$

From 90 to $95 \mathrm{~cm}$ depth $\left(44^{\circ} 47^{\prime} \mathrm{N}, 1^{\circ} 17^{\prime} \mathrm{E}\right)(11 / 30$ diluted sample). Pollen indicates last extension of Fagus, before beginning of extension of cultivation, expected during Sub-atlantic period.

\section{Ly-2359. Chanaleilles, Haute-Loire}

$2660 \pm 180$

From 65 to $70 \mathrm{~cm}$ depth $\left(44^{\circ} 51^{\prime} \mathrm{N}, 1^{\circ} 17^{\prime} \mathrm{E}\right)(7 / 15$ diluted sample). Pollen indicates, as for Ly-2359, beginning of last regression of Fagus.

\section{Ly.2357. Lestivalet I, 65 to 70, Malzieu-Forain, \\ Lozère}

$$
1360 \pm 160
$$

From 60 to $70 \mathrm{~cm}$ depth $\left(44^{\circ} 51^{\prime} \mathrm{N}, 1^{\circ} 13^{\prime} \mathrm{E}\right)(3 / 5$ diluted sample). Pollen indicates, as for Ly-2360, extension of Pinus and cereals. 


\section{Ly-2358. Lestivalet I, 125 to 130, Malzieu-Forain, Lozère \\ $8790 \pm 220$}

From 125 to $130 \mathrm{~cm}$ depth (1/2 diluted sample). Layer does not contain pollen, only charcoal. There seems to be sedimentation hiatus at overlying level.

Ly-2444. Lestivalet II, 146.5 to 154.5, MalzieuForain, Lozère

$11,330 \pm 280$

From 146.5 to $154.5 \mathrm{~cm}$ depth (11/30 diluted sample). Pollen indicates cold period with only $8 \%$ arboreal pollen.

\section{Ly-2445. Lestivalet II, 156.5 to 159.5, Malzieu-} Forain, Lozère

$7300 \pm 150$

From 156.5 to $159.5 \mathrm{~cm}$ depth (13/15 diluted sample). Pollen indicates temperate period with $15 \%$ arboreal pollen, mainly Betula and Salix.

General Comment (MR \& AP): 4 more recent dates agree with expected values (Sub-atlantic) (Reille \& Pons, 1982). Ly-2358 proves sedimentation hiatus occurred before Boreal. Ly-2444 is either too young or too old as it corresponds to generally accepted Alleröd age contrary to cold period indications by pollen; Ly-2445 is much too young for unknown reason.

\section{Massif de l'Aubrac series, Lozère}

Table 6 lists results from 2 borings in peat bogs at ca $1050 \mathrm{~m}$ alt at Brameloup near Recoules d'Aubrac $\left(44^{\circ} 43^{\prime} \mathrm{N}, 3^{\circ} 04^{\prime} \mathrm{E}\right)$ and at La Chaumette near Brion (44 $\left.44^{\circ} \mathrm{N}, 3^{\circ} 05^{\prime} \mathrm{E}\right)$. Coll 1979 and subm 1980 (Ly-2604 and -2605) by M Reille.

TABLE 6

Massif de l'Aubrac

\begin{tabular}{|c|c|c|c|c|c|}
\hline $\begin{array}{c}\text { Sample } \\
\text { no. }\end{array}$ & Boring & Depth & $\begin{array}{c}\text { Expected } \\
\text { climatic phase }\end{array}$ & $\begin{array}{l}\text { Dilution } \\
\text { ratio }\end{array}$ & Age BP \\
\hline Ly-2440 & Brameloup & $80-95 \mathrm{~cm}$ & Sub-atlantic & $3 / 10$ & $820 \pm 180$ \\
\hline-2441 & Brameloup & $135-170 \mathrm{~cm}$ & Sub-boreal & $1 / 2$ & $2660 \pm 190$ \\
\hline-2442 & Brameloup & $180-195 \mathrm{~cm}$ & Sub-boreal & $23 / 30$ & $4010 \pm 170$ \\
\hline-2443 & Brameloup & $230-245 \mathrm{~cm}$ & End of Atlantic & $1 / 10$ & $5770 \pm 480$ \\
\hline-2604 & Brameloup & $300-320 \mathrm{~cm}$ & Atlantic & $1 / 3$ & $6110 \pm 210$ \\
\hline-2605 & Brameloup & $410-435 \mathrm{~cm}$ & Beginning of Atlantic & $2 / 3$ & $6990 \pm 160$ \\
\hline-2110 & La Chaumette & $20-27 \mathrm{~cm}$ & Sub-atlantic & $1 / 2$ & $4300 \pm 180$ \\
\hline-2111 & La Chaumette & $55-62 \mathrm{~cm}$ & Sub-boreal & $2 / 5$ & $4670 \pm 190$ \\
\hline-2112 & La Chaumette & $142-150 \mathrm{~cm}$ & Beginning Atlantic & $3 / 5$ & $6880 \pm 200$ \\
\hline-2113 & La Chaumette & $177-185 \mathrm{~cm}$ & Eoreal & $1 / 3$ & $7980 \pm 260$ \\
\hline-2114 & La Chaumette & $292-300 \mathrm{~cm}$ & Pre-boreal & $1 / 8$ & $10,430 \pm 570$ \\
\hline-1857 & La Chaumette & $339-347 \mathrm{~cm}$ & Alleröd & $5 / 6$ & $10,910 \pm 360$ \\
\hline-1858 & La Chaumette & $403-413 \mathrm{~cm}$ & Bölling & 1 & $12,370 \pm 340$ \\
\hline-2115 & La Chaumette & $413-423 \mathrm{~cm}$ & Bölling & 1 & $11,490 \pm 170$ \\
\hline-2116 & La Chaumette & $423-433 \mathrm{~cm}$ & Bölling & $4 / 5$ & $12,810 \pm 250$ \\
\hline
\end{tabular}

General Comment (MR): Brameloup series agrees with palynol. Ly-2441 seems a little too young but may indicate sedimentation hiatus. Chaumette series is also consistent except Ly-2115 which looks ca $1000 \mathrm{yr}$ too young. Ly-1858 and -2116 are 1st 2 dates of Bölling period in Central Massif and correspond with beginning of Juniperus phase. 


\section{Peyrebeille series, La Villate, Ardèche}

Table 7 lists results obtained from peat from several levels in peat bog at $1265 \mathrm{~m}$ alt $\left(44^{\circ} 35^{\prime} \mathrm{N}, 3^{\circ} 58^{\prime} \mathrm{E}\right)$. Coll and subm 1979 by $\mathrm{M}$ Couteaux, Lab Palynol, Univ Marseille.

TABLE 7

Peyrebeille

\begin{tabular}{|c|c|c|c|c|c|c|}
\hline $\begin{array}{c}\text { Sample } \\
\text { no. }\end{array}$ & Boring & Depth & $\begin{array}{l}\text { Pollen } \\
\text { event }\end{array}$ & $\begin{array}{l}\text { Expected } \\
\text { climatic } \\
\text { phase }\end{array}$ & $\begin{array}{l}\text { Dilution } \\
\text { ratio }\end{array}$ & Age BP \\
\hline Ly-2203 & Peyrebeille I & $45-49 \mathrm{~cm}$ & $\begin{array}{l}\text { Beginning of } \\
\text { Abies } \text { extent }\end{array}$ & Sub-atlantic & 1 & $3310 \pm 120$ \\
\hline-2201 & Peyrebeille II & $52-58 \mathrm{~cm}$ & $\begin{array}{l}\text { Beginning of } \\
\text { Abies extent }\end{array}$ & Sub-atlantic & 1 & $3200 \pm 120$ \\
\hline-2204 & Peyrebeille I & $50-58 \mathrm{~cm}$ & $\begin{array}{l}\text { 2nd extent of } \\
\text { Fagus }\end{array}$ & Sub-boreal & $2 / 11$ & $4160 \pm 310$ \\
\hline-2202 & Peyrebeille II & $59-64 \mathrm{~cm}$ & $\begin{array}{l}\text { lst extent of } \\
\text { Fagus }\end{array}$ & Sub-boreal & 1 & $4360 \pm 130$ \\
\hline-2205 & Peyrebeille I & $58-66 \mathrm{~cm}$ & $\begin{array}{l}\text { lst extent of } \\
\text { Fagus }\end{array}$ & Sub-boreal & 1 & $3800 \pm 110$ \\
\hline-2206 & Peyrebeille I & $79-85 \mathrm{~cm}$ & $\begin{array}{l}\text { Before 1st } \\
\text { increase of } \\
\text { Fagus }\end{array}$ & $\begin{array}{l}\text { End of } \\
\text { Atlantic }\end{array}$ & $7 / 10$ & $4740 \pm 170$ \\
\hline-2207 & Peyrebeille I & $87-94 \mathrm{~cm}$ & & $\begin{array}{l}\text { End of } \\
\text { Atlantic }\end{array}$ & $1 / 2$ & $5340 \pm 190$ \\
\hline-2208 & Peyrebeille I & $105-118 \mathrm{~cm}$ & & $\begin{array}{l}\text { Beginning of } \\
\text { Atlantic }\end{array}$ & 1 & $5810 \pm 140$ \\
\hline-2209 & Peyrebeille I & $119.134 \mathrm{~cm}$ & & $\begin{array}{l}\text { Beginning of } \\
\text { Atlantic }\end{array}$ & 1 & $6910 \pm 140$ \\
\hline-2210 & Peyrebeille I & $139-143 \mathrm{~cm}$ & $\begin{array}{l}\text { lst max of } \\
\text { Quercus }\end{array}$ & Late Boreal & $3 / 5$ & $7240 \pm 170$ \\
\hline-2211 & Peyrebeille I & $143-146 \mathrm{~cm}$ & $\begin{array}{l}\text { lst max of } \\
\text { Corylus }\end{array}$ & Early Boreal & $1 / 2$ & $8550 \pm 240$ \\
\hline
\end{tabular}

General Comment (MC): dates agree well with expected range of values of palynol zones. Agreement between Ly-2203 and -2201 proves that Abies developed as early as end of Sub-boreal and not only during Sub-atlantic (Couteaux, 1978). Comparison of Ly-2202 and -2204 vs Ly-2205 shows that last result is ca $550 \mathrm{yr}$ too young.

\section{Lac de Siguret series, Saint-André d'Embrun, Hautes Alpes}

Table 8 lists results obtained from clay with organic matter from boring $78 \mathrm{SM}$ at ca $1000 \mathrm{~m}$ alt in lake sediments ( $\left.44^{\circ} 37^{\prime} \mathrm{N}, 6^{\circ} 33^{\prime} \mathrm{E}\right)$. Coll 1979 and subm 1981 by J L de Beaulieu.

TABLE 8

Lac de Siguret

\begin{tabular}{rlllr}
\hline $\begin{array}{c}\text { Sample } \\
\text { no. }\end{array}$ & Depth & $\begin{array}{c}\text { Expected } \\
\text { climatic phase }\end{array}$ & $\begin{array}{c}\text { Dilution } \\
\text { ratio }\end{array}$ & \multicolumn{1}{c}{ Age sP } \\
\hline Ly-2125 & $335-345 \mathrm{~cm}$ & Atlantic & $1 / 10$ & $7110 \pm 420$ \\
-2126 & $355-360 \mathrm{~cm}$ & Late Dryas & $1 / 15$ & $10,820 \pm 810$ \\
-2127 & $390-400 \mathrm{~cm}$ & Late Dryas & $2 / 5$ & $12,930 \pm 380$ \\
-2128 & $437-442 \mathrm{~cm}$ & Middle Dryas & $2 / 5$ & $13,540 \pm 350$ \\
-2129 & $460-468 \mathrm{~cm}$ & Middle Dryas & $1 / 2$ & $17,800 \pm 450$ \\
-2130 & $480-490 \mathrm{~cm}$ & Early Dryas & 1 & $17,410 \pm 220$ \\
-2131 & $504-520 \mathrm{~cm}$ & Würmian III & 1 & $20,770 \pm 620$ \\
\hline
\end{tabular}


General Comment (JL de B): previous results obtained by Louvain lab on same site was pub (de Beaulieu, 1977): upper levels attributed to the Subatlantic, from 215 to $220 \mathrm{~cm}, \mathrm{Lv}-709: 2920 \pm 70$ and to Sub-boreal, from 275 to $290 \mathrm{~cm}, \mathrm{Lv}-710: 3660 \pm 75$. Another result from deeper level, 430 to $440 \mathrm{~cm}, \mathrm{Lv}-712: 13,190 \pm 260$ fits well with Ly-2128 from same depth. However, all values except Ly-2125 and -2128 which are in expected date range because of large statistical margins, are too old for palynol data: Ly-2127 seems ca 2000 yr too old, Ly-2128 (Lv-712) ca 1000 yr, and Ly2129, at least $4000 \mathrm{yr}$. In comparison with Pelléautier profile (below; R, 1979, v 21, p 414-416) Ly-2130 should also be ca $3000 \mathrm{yr}$ too old while rather old date of Ly-2131 remains questionable.

\section{Pelléautier series, Hautes Alpes}

Gray clay from two deep layers in "La Motte-qui-Tremble" peat bog

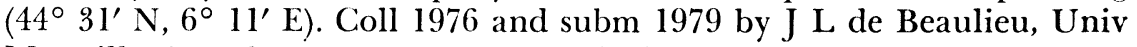
Marseille. Samples subm to check relatively old age obtained from lowest level of 18 results of previously pub series (R, 1979, v 21, p 414-416).

\section{Ly-1942. VI, 670-678cm}

Clay with very little organic matter. (7/60 diluted sample)

\section{Ly-1943. VI, 678-690cm}

Brown clay. (3/7 diluted sample)

General Comment (JL de B): three dates of previous series dated 600 to $635 \mathrm{~cm}$ level between 14,500 and $15,500 \mathrm{BP}$, close to Ly-1943; but Ly1796: $23,700+1900$ previously obtained for $700 \mathrm{~cm}$ level also fits with Ly-1942. Thus, because of lack of more data, discrepancy between Ly-1942 and -1943 remains unexplained and fairly old age of Ly-1796 is still questionable.

\section{Haut Dauphiné series, Isère}

Tables 9 and 9A list results of measurements of peat and clay from several levels of borings in high alt peat bog ponds. Coll by M Couteaux.

TABLE 9

Haut Dauphiné

\begin{tabular}{|c|c|c|c|c|}
\hline Sites & Village & Alt & Loc & Colln yr \\
\hline Vallon de Lavey & $\begin{array}{l}\text { La Muande, St-Christophe } \\
\text { en Oisan }\end{array}$ & $2050 \mathrm{~m}$ & $\left(44^{\circ} 58^{\prime} \mathrm{N}, 6^{\circ} 13^{\prime} \mathrm{E}\right)$ & 1980 \\
\hline La Tourbière & Muzelle, Vénosc & $2140 \mathrm{~m}$ & $\left(44^{\circ} 57^{\prime} \mathrm{N}, 6^{\circ} 06^{\prime} \mathrm{E}\right)$ & 1978 \\
\hline Draye de Pertu & $\begin{array}{l}\text { Les Etages, St-Christophe } \\
\text { en Oisan }\end{array}$ & $1590 \mathrm{~m}$ & $\left(45^{\circ} 53^{\prime} \mathrm{N}, 6^{\circ} 15^{\prime} \mathrm{E}\right)$ & 1980 \\
\hline Côte Brune & $\begin{array}{l}\text { Les Deux Alpes, Mont } \\
\text { de Lans }\end{array}$ & $1646 \mathrm{~m}$ & $\left(45^{\circ} 00^{\prime} \mathrm{N}, 6^{\circ} 07^{\prime} \mathrm{E}\right)$ & 1980 \\
\hline
\end{tabular}


TABLE 9A

\begin{tabular}{|c|c|c|c|c|c|}
\hline $\begin{array}{l}\text { Sample } \\
\text { no. }\end{array}$ & Boring & Depth & $\begin{array}{c}\text { Expected } \\
\text { climatic phase }\end{array}$ & $\begin{array}{l}\text { Dilution } \\
\text { ratio }\end{array}$ & Age вP \\
\hline Ly-2374 & Muande I-B & $35-39 \mathrm{~cm}$ & End of Sub-boreal & $2 / 5$ & $2320 \pm 190$ \\
\hline-2375 & Muande I-A & $51-56 \mathrm{~cm}$ & End of Sub-boreal & $8 / 15$ & $2570 \pm 170$ \\
\hline-2376 & Muande I-A & $71-75 \mathrm{~cm}$ & End of Sub-boreal & $13 / 30$ & $5150 \pm 200$ \\
\hline-2394 & Muzelle I-808 & $280-290 \mathrm{~cm}$ & Sub-atlantic & $4 / 5$ & $2060 \pm 130$ \\
\hline-2395 & Muzelle I-809 & $305-315 \mathrm{~cm}$ & Sub-boreal & 1 & $3040 \pm 120$ \\
\hline-2396 & Muzelle I-8010 & $345-355 \mathrm{~cm}$ & Sub-boreal & $11 / 15$ & $4440 \pm 180$ \\
\hline-2397 & Muzelle I-8011 & $370-380 \mathrm{~cm}$ & Atlantic & 1 & $5320 \pm 140$ \\
\hline-2402 & Muzelle I-8012 & $665-670 \mathrm{~cm}$ & $\begin{array}{l}\text { Beginning of } \\
\text { Atlantic }\end{array}$ & 1 & $8430 \pm 150$ \\
\hline-2403 & Muzelle I-8013 & $680-684 \mathrm{~cm}$ & Boreal & $3 / 5$ & $10,540 \pm 190$ \\
\hline-2404 & Muzelle I-8014 & $684-690 \mathrm{~cm}$ & Beginning of Boreal & $4 / 5$ & $10,410 \pm 200$ \\
\hline-2398 & Muzelle II-804 & $490-500 \mathrm{~cm}$ & Boreal & $14 / 15$ & $8420 \pm 160$ \\
\hline-2399 & Muzelle II-805 & $505-511 \mathrm{~cm}$ & Boreal & 1 & $9480 \pm 180$ \\
\hline-2400 & Muzelle II-806 & $519-527 \mathrm{~cm}$ & Beginning of Boreal & $14 / 15$ & $10,920 \pm 200$ \\
\hline-2401 & Muzelle II-807 & $536-542 \mathrm{~cm}$ & Late Dryas & $3 / 10$ & $13,460 \pm 390$ \\
\hline-2148 & Draye de Pertu I & $6 \mathrm{~m}$ & Late Dryas & $1 / 3$ & $7130 \pm 240$ \\
\hline-2146 & Côte Brune & Top & & 1 & $12,310 \pm 150$ \\
\hline-2147 & Côte Brune & Base & & 1 & $12,890 \pm 180$ \\
\hline
\end{tabular}

General Comment (MC): comparison between results from La Muande neighboring borings for same pollen event establishes boundary between Sub-boreal and Sub-atlantic periods when Pinus mugo Torr gave way to Pinus cembra L. This boundary is rarely seen in pollen diagrams from high alt sites (Couteaux, 1981). At La Muzelle, dates are older than expected for pollen phases, which may be explained by presence of carbonaceous secondary carboniferous sediments in samples. La Draye de Pertu date differs from expected one. Dated level actually was Atlantic fire level, sparse remaining pollen of which wrongly suggested cold climate vegetation. Both La Côte Brune dates are, as expected, before Alleröd but are too young because of introduction of roots (Couteaux and Evin, 1981).

\section{B. Bone samples from grottoes}

\section{Ly-2171. Ours de Forsyth-Major, Monte Estremo, \\ Corsica}

$200 \pm 90$

Bone fragments from ribs of bear skeleton, coll 1906 by C Forsyth Major in Inferno grotto $\left(42^{\circ} 22^{\prime} \mathrm{N}, 8^{\circ} 49^{\prime} \mathrm{E}\right)$. Preserved since colln in Paleontol Lab, Mus Hist Nat Paris and subm 1979 by F Poplin, Paris. Comment (FP): despite apparently young aspect of bones (Forsyth-Major, 1930) skeleton was believed to represent presence of bears in Corsica early in Pleistocene times. Date disproved this and demonstrated that animal was brought onto island by man.

\section{Ly-2311. Le Mont Terret, Vallée du Perthuis, Thorens- Glières, Haute Savoie \\ $1280 \pm 150$ \\ Ursus arctos bones from small grotto that opens onto slope of $\mathrm{mt}$ $\left(45^{\circ} 57^{\prime} \mathrm{N}, 6^{\circ} 15^{\prime} \mathrm{E}\right)$. Coll 1979 by G Fontana, Belley; studied and subm 1980 by R Ballesio, Geol Dept, Univ Lyon (29/30 diluted sample). Com- ment (RB): date suggests relatively recent age for brown bears in Préalps}


$\mathrm{mts}$, while they only disappear from high part of W Alps at beginning of 20th century.

Ly-1805. Grottes Glacée, Bechloul, Bouira, Algeria $9620 \pm 200$

Bear bones from clayey fill of "Grotte Glacée" $\left(36^{\circ} 30^{\prime} \mathrm{N}, 4^{\circ} 00^{\prime} \mathrm{E}\right)$. Coll 1977 by P Gillon and P E Coiffait, Univ Constantine, and subm 1978 by G Auboire, Joinville, France. Comment (GA): paleontol study still in process will probably confirm bear is Ursus spelaeus, who presumably disappeared from Algeria during Neolithic. Date is compatible with this hypothesis and attributes Holocene age to grotto filling.

\section{Grotte Zawalona series, Mnikow, Krakow Province, Poland}

Bone of large mammifera from last loess layer mixed with cryoclastic rock rubble of Zawalona grotto $\left(51^{\circ} 52^{\prime} \mathrm{N}, 19^{\circ} 40^{\prime} \mathrm{E}\right)$. Coll and subm 1978 by J L Kozlowski, Inst Archaeol, Univ Jagellon, Krakow.

$\begin{array}{lll}\text { Ly-2270. Top } & 14,060 \pm 340\end{array}$

From top of layer, just underlying uncharacteristic industry (probably Magdalenian); result is average of two measurements on $2 / 3$ and $14 / 15$, respectively, diluted samples.

Ly-2271. Base

$15,380 \pm 340$

From base of layer, assoc with uncharacteristic Gravettian industry.

General Comment (JKK): both dates offer precise chronology to series of loess and cryoclastic sediments very often found in fill of Polish grottoes; period following main phase of loess sedimentation may be dated to 16,000 or $15,000 \mathrm{BP}$. This questions hypothesis of contemporaneity between late upper loess and loessic and cryoclastic upper sediments of Polish grottoes.

\section{Ly-2277. Aven Bouët, Les Matelles, Hérault}

$15,460 \pm 380$

Bones from fill of karstic fossil system $\left(43^{\circ} 44^{\prime} \mathrm{N}, 3^{\circ} 49^{\prime} \mathrm{E}\right)$. Coll 1979 by J P Brigal and subm 1980 by J L Vernet, Univ Montpellier. Assoc with expected Late Pleistocene fauna containing small feline resembling present-day lion. Comment (JLV): date confirms faunal attribution to Late Würmian period.

\section{Ly-2416. Aven des Cervidés, Cournonterral,}

Hérault

Bones (Cervus elephas) from surface of clayey filling in bottom of Les Cervidés aven $\left(43^{\circ} 41^{\prime} \mathrm{N}, 3^{\circ} 41^{\prime} \mathrm{E}\right)$. Coll 1978 by X Gutherz and subm 1980 by A Bonnet, Nîmes. Assoc with fauna of great red deer, horse, bovine, and small capridae, probably from Würmian interstadial or postglacial period. (1/3 diluted sample). Comment (AB): dates fauna to Würmian IV when climate fitted well with such fauna.

\section{Ly-2452. Grotte des Bisons, Lurbe-Saint-Christau, Pyrénées Atlantiques}

Bones from upper level of fill of grotto, small cavity in karstic system $\left(43^{\circ} 07^{\prime} \mathrm{N}, 0^{\circ} 35^{\prime} \mathrm{W}\right)$. Coll 1977 and subm 1981 by $\mathrm{G}$ Marsan, Inst 
Quaternaire, Univ Bordeaux. (3/10 diluted sample). Comment (GM): dates fauna to Late Würmian with dominance of Bison priscus, Equus caballus, and presence of Ursus spelaeus, Rangifer tarandus, and Rupicapra rupicapra, which also fits paleontol data.

Ly-2102. Grotte de Bos, Caniac du Causse, Lot $\quad 21,460 \pm 480$

Bones from 15 upper $\mathrm{cm}$ of gallery fill in small grotto $\left(44^{\circ} 38^{\prime} \mathrm{N}, 1^{\circ}\right.$ $40^{\prime} \mathrm{E}$ ). Coll 1978 and subm 1980 by R Séronie-Vivien, Le Bouscat, Gironde. No assoc industry. Comment (RS-V): no definite age was expected, but date agrees with value obtained for base of Layer $9 \mathrm{~b}$ in Pégourié site, Ly-1835: 24,200 \pm 1100 (below) and with relatively recent ages previously found in numerous karstic fills of Causse de Gramat region (Philippe, Mourer, \& Evin, 1981).

\section{Ly.2415. La Baume Longue, Dions, Gard}

$26,500 \pm 1000$

Bones (Ursus spelaeus) from base of fill in bottom of "Grand Puits" pit $\left(43^{\circ} 56^{\prime} \mathrm{N}, 4^{\circ} 18^{\prime} \mathrm{E}\right)$. Coll 1970 and subm 1980 by A Bonnet. Assoc with Ursus spelaeus and Crocuta spelaea probably from Early Würmian. Dates confirm previous measurements from La Sartanette site (Ly-1591: 22,700 \pm 1700 and Ly-1590: 21,900 $\pm 1500, R, 1979, v 21, p$ 418-419) and from Grotte Latrone (Ly-1966, below) and suggest that cavern bear only disappeared during last part of Würmian in Europe.

\section{Ly-225!. Grotte du Castellas, Dourgne, Tarn $\quad \mathbf{2 6 , 4 0 0} \pm \mathbf{7 0 0}$}

Bones from one of filled levels $\left(43^{\circ} 29^{\prime} \mathrm{N}, 2^{\circ} 09^{\prime} \mathrm{E}\right)$. Coll 1979 by P M de la Morsanglière and subm 1980 by $F$ Prat, Univ Bordeaux. Assoc with tall mammals and Upper Paleolithic industry. (13/15 diluted sample). Comment (PMdelaM): dates sediments to Würmian III age in agreement with fauna and archaeol.

\section{Grotte de Bourdette series, Sainte-Colombe en Bruilhois, Lot et Garonne}

Bones from several levels $\left(44^{\circ} 12^{\prime} \mathrm{N}, 0^{\circ} 24^{\prime} \mathrm{W}\right)$. Coll 1979 by $\mathrm{J}$ Chagneau and subm 1980 by F Prat.

\section{Ly-2345. Couche 1a}

From Layer la under 1 to $2.5 \mathrm{~cm}$ of clay, presumably from Middle or Late Würmian. (13/15 diluted sample)

Ly-2346. Couche 3 inf

$30,300 \pm 1200$

From base of Layer 3, presumably Middle Würmian. (17/30 diluted sample)

\section{Ly.2347. Couche 8}

$30,400 \pm 1000$

From Layer 8 at ca $2 \mathrm{~m}$ deeper than Layer 1a, presumably from Early Würmian. (23/30 diluted sample)

General Comment (JC): three dates attribute fill to same age. Some detected activity suggests contamination, origin of which remains unknown because of overlying clavey sediment. Series could be considered min age 
of $\geqslant 29,000 \mathrm{BP}$; the only certain conclusion should be that grotto fill was deposited before Würmian III period.

Ly-1966. Grotte de Latrone, Sainte Anastasie, Gard $29,600 \pm 1100$

Bone (Ursus spelaeus) from soil of gallery at bottom of pit at Russan, $\left(43^{\circ} 56^{\prime} \mathrm{N}, 4^{\circ} 20^{\prime} \mathrm{E}\right)$. Coll 1948 by $\mathrm{R}$ Jeantet, preserved in mus and subm 1978 by A Bonnet. Expected date: Early Würm. Comment (AB): dates sample to beginning of Würm III and agrees with previous measurement from neighboring site, La Sartanette, Ly-1591: 22,700 вP \pm 1700, (R, 1979, v 21, p 48) and from Baume Longue at Dions, Ly-2415 (above). Results confirm Ursus spelaeus is present at least up to late Würmian.

Ly-2309. Grotte du Coustal, Noailles, Corrèze $\geqslant \mathbf{3 0 , 0 0 0}$

Bones from fill of Coustal grotto karstic system $\left(45^{\circ} 05^{\prime} \mathrm{N}, 1^{\circ} 20^{\prime} \mathrm{E}\right)$. Coll 1980 by J P Raynal and subm 1981 by M Philippe, Mus Hist Nat Lyon. Assoc with presumed Rissian fauna which may also be Würmian. (1/3 diluted sample). Comment (MP): date confirms lst attribution; it is not Late Würmian and agrees with many other results in Causse de Martel calcareous region (Philippe, Mourer, \& Evin, 1981).

\section{Ly-2278. Gouffre de Moustayous, Saint-Pé de Bigorre, Hautes Pyrénées}

Bones (Lynx lynx) from surface of gallery fill in karstic system $\left(43^{\circ}\right.$ $\left.04^{\prime} \mathrm{N}, 0^{\circ} 10^{\prime} \mathrm{W}\right)$. Coll and subm 1980 by A Clot, Bordères sur Echez, Hautes Pyrénées. (1/6 diluted sample). Comment (AC): bone does not belong to Late Würmian (Clot, 1982).

\section{Samples from fluviatile sediments}

Ly-2190. Berge du File, Milly-Lamartine, Saône et Loire

$$
\Delta^{14} \mathrm{C}=-\mathbf{2 . 2} \begin{array}{r}
\text { Modern } \\
\mathbf{1 . 6} \%
\end{array}
$$

Wood from lowest level of sediment series from side of Le File Stream $\left(46^{\circ} 21^{\prime} \mathrm{N}, 4^{\circ} 43^{\prime} \mathrm{E}\right)$. Coll 1979 and subm 1980 by A J Argant, Bron. Comment (AJA): date confirms sediment series probably is fill of mill reservoir. Deposit consists of alternating pebbly and clayey sediments overlying rubble; series suggests postglacial deposit overlying Late glacial sediment. ${ }^{14} \mathrm{C}$ date negates need for pollen analyses.

\section{Ly-2299. Larche, Corrèze \\ Modern} Vézère R $\left(45^{\circ} 8^{\prime} \mathrm{N}, 1^{\circ} 26^{\prime} \mathrm{E}\right)$. Coll 1979 by P Y Demars and subm 1980 by J P Raynal, Inst Quaternaire, Univ Bordeaux. Measured to date formation of upper part of terrace. Comment (JPR): younger than expected: date only indicates redeposition of sediments and does not give max age to 1st deposition of alluvia on top of terrace.

\section{Bernalda and Pomarico series, Basilicates, Italy}

Charcoal from paleosoils in sands of alluvia of coastal rivers between Bernalda and Pomarico near Matera ( $\left.40^{\circ} 26^{\prime} \mathrm{N}, 16^{\circ} 39^{\prime} \mathrm{E}\right)$. Coll and subm 1978 by $S$ Tazioli, Univ Bari, Italy. 


\section{Ly.1852. Paléosol supérieur}

$840 \pm 190$

From $4 \mathrm{~m}$ depth.

\section{Ly.1851. Paléosol inférieur}

$1550 \pm 350$

From $9 \mathrm{~m}$ depth. (11/20 diluted sample)

General Comment (ST): both dates agree with expected age of assoc potsherds (Nébois, 1974) and indicate sedimentation rate of alluvia.

\section{Villers-le-Lac series, Doubs}

Vegetal remains coll by borings from four levels in deep alluvia lying in local deepening in Le Doubs $\mathrm{R}$ valley, upstream from Chaillexon Lake $\left(47^{\circ} 04^{\prime} \mathrm{N}, 6^{\circ} 40^{\prime} \mathrm{E}\right)$. Coll and subm 1977 by M Campy, Dept Géol, Univ Besançon. (9/10 diluted sample for Ly-2027 and -2028).

$$
\begin{array}{lll}
\text { Ly-2025. } & 13.75 \mathrm{~m} & 2970 \pm 130 \\
\text { Ly-2026. } & 15.5 \mathrm{~m} & 3130 \pm 120 \\
\text { Ly-2027. } & 18.5 \mathrm{~m} & 3220 \pm 170 \\
\text { Ly.2028. } & 23.5 \mathrm{~m} & 4000 \pm 160
\end{array}
$$

General Comment (MC): ages in larger time range were expected. Closer and relatively recent ages obtained prove large Holocene filling, due to rapid lacustrine sedimentation after slide of cliffs into valley (Campy, $1980)$.

\section{Anglefort series, Ain}

Wood from ca $14 \mathrm{~m}$ depth in Rhône $\mathrm{R}$ alluvia, found during laying of foundation of generating sta $\left(45^{\circ} 55^{\prime} \mathrm{N}, 5^{\circ} 50^{\prime} \mathrm{E}\right)$. Coll 1978 by Co Natle Rhône (CiNR) and subm 1978 by R Vilain (Ly-1976 and -1977), Dept Géol, Univ Lyon, and 1980 by CNR (Ly-2187).

$$
\begin{array}{lll}
\text { Ly.1976. } & \text { No. } 1 & 2890 \pm 150 \\
\text { Ly.2187. } & \text { CNR } & 3550 \pm 120 \\
\text { Ly.1977. } & \text { No. } 6 & 6090 \pm 160
\end{array}
$$

General Comment (RV): Ly-1976 is very close to Ly-135: $2880 \pm 220$ (R, 1971 , v 13, p 55), from "Chêne de la Balme" wood found 20km downstream in same alluvia. Expected range of dates was older because of depth. Differences among three samples prove that several deposits of flattened wood occurred at same loc because of meandering main channel in alluvial plain.

Ly-1961. Sion, Valais, Switzerland $3650 \pm 140$

Wood from $16 \mathrm{~m}$ depth in alluvia of Rhône $\mathrm{R}$ valley at alt $480 \mathrm{~m}\left(46^{\circ}\right.$ $14^{\prime} \mathrm{N}, 7^{\circ} 20^{\prime} \mathrm{E}$ ). Coll by M Eschbach and subm 1979 by A Bezinge, Sté Grande Dixence, Sion. Comment (AB): dates rapid filling of alluvial valley; many assoc artifacts from Roman times. 


\section{Blanchon series, Saint-Jean-le-Vieux, Ain}

Wood from sandy layer embedded in alluvia of Ain $\mathrm{R}$ valley $\left(46^{\circ} \mathbf{0 3}^{\prime}\right.$ $\mathrm{N}, 6^{\circ} 21^{\prime} \mathrm{E}$ ). Coll and subm 1978 by A Billard, Inst Geog, Univ Paris.

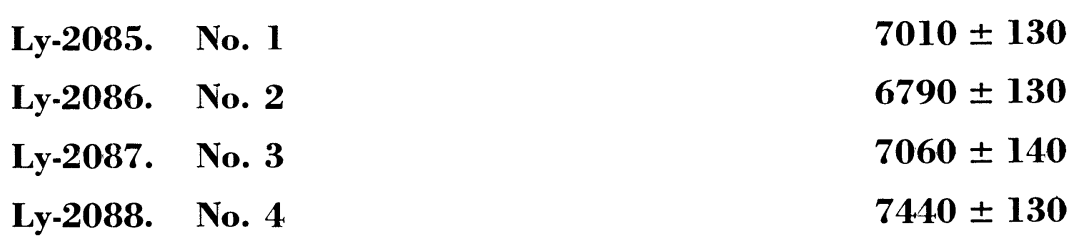

General Comment (AB): similar ages of four samples indicate homogeneous deposit. They agree with expected Holocene age of embedding alluvia and show that lowest terrace of river cannot be older than Atlantic period.

Ly-2001. Le Fontanil, Isère

$9900 \pm 250$

Charcoal from single layer of vegetal material in alluvia of dejection

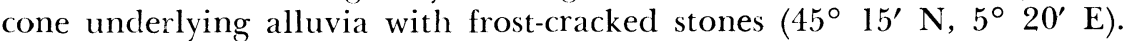
Cioll 1969 by M Colardelle and subm 1969 by A Bosquet, Centre Documentation Préhist Alpine, Grenoble. Comment (AB): at sampling, embedding sediment was thought to be from interstadial and expected deduced age $>30,000 \mathrm{yr}$. Date is much younger but still possible if overlying sediment was deposited cluring very cold phase of Late Dryas period.

\section{Le: Torrents du Bochaine series, Hautes-Alpes}

Wood from tree trunks rooted in silt and gravel of sloping banks of Bochaine region, Le Buëch R basin, near Aspres-sur-Buëch. Coll 1977 and subm 1978 by M Archambault, Geog Dept, Univ Orléans. Samples listed in table 10.

TABLE 10

Les Torrents du Bochaine

\begin{tabular}{|c|c|c|c|c|c|}
\hline $\begin{array}{c}\text { Sample } \\
\text { no. }\end{array}$ & Valley & Village & Loc & Depth & Age BP \\
\hline Ly-1900 & $\begin{array}{l}\text { Torrent des } \\
\text { Richardets }\end{array}$ & $\begin{array}{l}\text { St Auban } \\
\text { d'Oze }\end{array}$ & $\left(44^{\circ} 30^{\prime} \mathrm{N}, 5^{\circ} 51^{\prime} \mathrm{E}\right)$ & $-7 \mathrm{~m}$ & $3790 \pm 140$ \\
\hline-1901 & Torrent de & St Pierre & & & \\
\hline & Bourdoutane & d'Argençon & $\left(44^{\circ} 31^{\prime} \mathrm{N}, 5^{\circ} 43^{\prime} \mathrm{E}\right)$ & $-2.5 \mathrm{~m}$ & $7150 \pm 260$ \\
\hline-1899 & Torrent Bachassette & Oze & $\left(44^{\circ} 31^{\prime} \mathrm{N}, 5^{\circ} 48^{\prime} \mathbf{E}\right)$ & $-8 m$ & $8820 \pm 240$ \\
\hline-1902 & Torrent Barnèche & Le Saix & $\left(44^{\circ} 29^{\prime} \mathrm{N}, 5^{\circ} 48^{\prime} \mathrm{E}\right)$ & $-5 \mathrm{~m}$ & $10,040 \pm 260$ \\
\hline
\end{tabular}

General Comment (MA): last three results agree well with many other tree trunks in region (Archambault, 1967). They all confirm Holocene age of youngest sloping banks. Ly-1900 (R, 1973, v 15, p 516) is younger than expected and may correspond with phase of detrital accumulation after sloping banks' edification period.

\section{Muret series, Haute-Garonne}

Wood from $4 \mathrm{~m}$ depth in alluvia of lowest terrace of La Garonne $\mathrm{R}$ $3 \mathrm{~km} \mathrm{SW}$ of Muret $\left(43^{\circ} 27^{\prime} \mathrm{N}, 1^{\circ} 18^{\prime} \mathrm{E}\right)$. Coll and subm 1979 by J C Revel, Lab Pédol, Univ Toulouse. 
General Comment (JCR): both dates confirm chronol homogeneity of sediments and rapid sedimentation rate in valley as samples come from distance of $1.5 \mathrm{~km}$ of actual river bed. They also confirm Holocene age attributed to terrace and are close to other results from sample found in same alluvia ca $150 \mathrm{~km}$ downstream at Golfech near Valence d'Agen, Gif2338: $8900 \pm 160(\mathrm{R}, 1974, \mathrm{v} 16, \mathrm{p} 62)$.

\section{Pugère du Rocher series, Sénas, Bouches du Rhône}

Samples from several levels in alluvial cone lying on one of terraces of La Durance R ( $\left.43^{\circ} 45^{\prime} \mathrm{N}, 5^{\circ} 13^{\prime} \mathrm{E}\right)$. Coll 1976 by G Clauzon, Inst Geog, Univ Aix-en Provence.

\section{Ly-1917. A-142}

$10,440 \pm 460$

Bones from upper level of cone, assoc with Epipaleolithic industry (Escalon de Fonton, 1976). (1/3 diluted sample)

\section{Ly-1972. A-142 bis}

Small bits of charcoal scattered in same levels as Ly-1917.

\section{Ly-2320. A-231} sample)

Terrestrial gastropod shells from lower level of cone. (1/10 diluted

General Comment (GC): Ly-1917 agrees with age of Montadian (Epipaleolithic) assoc industry attributed by previous measurement on sample from Layer 3 of neighboring La Montagne site (Escalon de Fonton, 1976), MC-1159: $9000 \pm 100$. This value also dates end of cone formation while Ly-1972 proves redeposit of ancient materials previously dated at Vautubières to ca 31,000 BP, Ly-769 (R, 1975, v 17, p 9) and Ly-1002 (R, 1976, $\mathrm{v} 18, \mathrm{p} 65)$. Ly-2320 gives min age for bottom of alluvial cone and therefore to River terrace, as terrace was deposited after La Durance capture by Le Rhône R; Ly-2320 also proves that capture occurred before Late Würmian.

Ly-2103. Polignac, Haute-Loire

$33,000 \pm 1000$

Horse bones $\left(45^{\circ} 4^{\prime} \mathrm{N}, 3^{\circ} 52^{\prime} \mathrm{E}\right)$. Coll 1979 by R Séguy, Le Puy, and subm 1979 by J P Raynal, Inst Géol Quaternaire, Univ Bordeaux. No assoc industry. (2/3 diluted sample). Comment (JPR): comparable with Ly-1988 (below) from lowest levels of Les Riveaux site in which same horse was discovered. Both dates agree well with expected Würmian III.

\section{Ly-1988. Les Rivaux Loc 1 base, Espaly-Saint- 30,600 $+1600$ Marcel, Haute-Loire $-1300$}

Horse bones from base of B unit, Levels 312 and 316, Loc 1 , $\left(45^{\circ} 3^{\prime}\right.$ N, $3^{\circ} 51^{\prime}$ E). Coll 1977 by J P Raynal and subm 1978. Comment (JPR): 
date agrees with sedimentol, paleontol, and archeol data which attribute Würmian III age to base of Unit B (Raynal et al, 1980).

\section{Erquighem-sur-la-Lys series, Nord}

Silt with vegetal debris from several levels of boring in deep alluvia of La Lys R $\left(50^{\circ} 40^{\prime} \mathrm{N}, 2^{\circ} 50^{\prime} \mathrm{E}\right)$. Coll 1977 by Bur Recherches Géol Min and subm 1979 by J Sommé, Univ Lille.

\section{Ly-2029. 46}

$\mathbf{2 0 , 6 4 0} \pm \mathbf{7 5 0}$

From 7.5 to $7.75 \mathrm{~m}$ depth. Pollen diagram indicates $40 \%$ of arboreal pollen with dominance of Pinus and presence of Corylus, Alnus, and Picea. Attributed to Weichselian interstadial. (1/3 diluted sample)

Ly-2030. 45

$24,000 \pm 600$

From 7.8 to $7.95 \mathrm{~m}$ depth. Pollen diagram indicates $20 \%$ arboreal pollen with dominance of Pinus and Corylus and presence of Alnus. Attributed to Weichselian interstadial. (2/3 diluted sample)

From 13.5 to $13.75 \mathrm{~m}$ depth. Pollen diagram indicates $90 \%$ arboreal pollen with dominance of Corylus and presence of Alnus, Quercus, Ulmus, and Fraxinus. Attributed to 2nd part of Middle Eemian interglacial. (9/10 diluted sample)

Ly-2032. 26

$29,000 \pm 700$ Ly-2031.

From 14 to $14.25 \mathrm{~m}$ depth. Pollen diagram indicates same data as

General Comment (JS): Ly-2031 and -2032 must be considered min ages, detected low activity from contamination not eliminated by chemical pretreatment. Ly-2029 and -2030 agree with Weichselian attribution, but slightly older dates corresponding to interstadials generally dated from 40,000 to 30,000 BP were expected. Some contamination may be present.

\section{Samples from various continental sediments}

\section{Massif forestier d'Osseja series, Pyrénées Orientales}

Charcoal from sub-surface sediments in SE Osseja $\left(42^{\circ} 22^{\prime} \mathrm{N}, 2^{\circ} 07^{\prime}\right.$ E). Coll by J N Puig and subm 1980 by J L Vernet, Univ Montpellier, to date min age of underlying soil, to evaluate age of colluvia contemporaneous with charcoal, and to establish evolution of vegetation. Table 11 lists results.

TABLE 11

Massif forestier d'Osseja

\begin{tabular}{ccccr}
\hline $\begin{array}{c}\text { Sample } \\
\text { no. }\end{array}$ & $\begin{array}{c}\text { Sample } \\
\text { ref }\end{array}$ & $\begin{array}{c}\text { Colln } \\
\text { date }\end{array}$ & $\begin{array}{c}\text { Dilution } \\
\text { ratio }\end{array}$ & \multicolumn{1}{c}{ Age BP } \\
\hline Ly-2412 & 80026 Le Puig & 1980 & $1 / 3$ & $280 \pm 190$ \\
-2413 & 80022 Couronnes & 1980 & $1 / 6$ & $1380 \pm 240$ \\
-2414 & 8195 Rhodoraie & 1978 & $2 / 5$ & $1410 \pm 180$ \\
\hline
\end{tabular}


General Comment (JLV): dates prove that different periods of clearing of sub-alpine and mountainous forests occurred from 4th to 8th centuries and from 15 th and 19th centuries.

\section{Forêt domaniale de Bédoin series, Vaucluse}

Very small amount of charcoal from several levels of pedologic profile at $820 \mathrm{~m}$ alt in Bédoin forest $\left(44^{\circ} 09^{\prime} \mathrm{N}, 5^{\circ} 13^{\prime} \mathrm{E}\right)$. Coll and subm 1978 by M Thinon, Lab Bot, Marseille. Table 12 lists results.

TABLE 12

Forêt domaniale de Bédoin

\begin{tabular}{ccccc}
\hline $\begin{array}{c}\text { Sample } \\
\text { no. }\end{array}$ & $\begin{array}{c}\text { Sample } \\
\text { ref }\end{array}$ & Depth & $\begin{array}{c}\text { Dilution } \\
\text { ratio }\end{array}$ & Age BP \\
\hline Ly-1693 & Bédoin 3 & $30 \mathrm{~cm}$ & $1 / 10$ & $710 \pm 500$ \\
-1692 & Bédoin 2 & $50 \mathrm{~cm}$ & $1 / 10$ & $1540 \pm 470$ \\
-1691 & Bédoin 1 & $1 \mathrm{~m}$ & $1 / 6$ & $1830 \pm 440$ \\
\hline
\end{tabular}

General Comment (MY): despite large uncertainty margins due to small amounts of available material, three results confirm botanic study of charcoal which suggests recent anthropogenic deforestation and substitution of Quercus sp and Taxus baccata by Quercus ilex (Thinon, 1978).

Ly-2000. Bois du Spitzberg

Fragment of black tree trunk from Spitzberg $\left(78^{\circ} 00^{\prime} \mathrm{N}, 17^{\circ} 00^{\prime} \mathrm{E}\right)$. Coll 1965 by J Corbel, Caluire, preserved in ${ }^{14} \mathrm{C}$ lab and measured 1978. Comment: date is comparable with values often found on such ice-floating wood which probably come far from S continent, see eg, Lu-24l: $2650 \pm$ 55 вр from Adventdalen, Spitzberg (R, 1970, v 12, p 546).

\section{Ly-1960. Glacier de Tzeudet, Valais, Switzerland $\quad 8110 \pm 180$}

Fragment of tree trunk found at $2460 \mathrm{~m}$ alt on moraine surface of Tzeudet glacier, on slope of Vélan Mt near Bourg-Saint-Pierre $\left(45^{\circ} 53^{\prime} \mathrm{N}\right.$, $\left.7^{\circ} 11^{\prime} \mathrm{E}\right)$. Coll by M May and subm 1979 by A Bezinge, Sté Grande Dixence, Sion. Comment (AB): date corresponds well with many other measurements on moraine wood from region, eg, from Arolla glacier, Ly-749: $8400 \pm 200$; Z'Mutt glacier, Ly-681: $7590 \pm 180$ (both in R, 1975, v 17, p 7, 8); Gorner glacier, Ly-298: $8160 \pm 220$ (R, 1972, v 15, p 135). These dates indicate very high uplift of timber line at end of Boreal period (Vivian, 1975; Bezinge \& Vivian, 1976).

\section{Ly-2294. Glacier de Thorens, Saint-Martin-de- \\ Belleville, Savoie}

$3920 \pm 100$

Fragment of tree trunk from $2200 \mathrm{~m}$ alt in Thorens glacial moraine $\left(45^{\circ} 22^{\prime} \mathrm{N}, 6^{\circ} 30^{\prime} \mathrm{E}\right)$. Coll and subm 1980 by $\mathrm{R}$ Vivian, Inst Geog Alpine, Univ Grenoble. Comment (RV): date agrees with those of similar trees of Belleville region at alt $>2000 \mathrm{~m}$, which is presently above timber line. Dates indicate that forest grew at this alt during end of Atlantic period and probably disappeared at beginning of Sub-boreal. 


\section{Creissels series, Aveyron}

Calcareous tufa from two tufa cliffs rising above Tarn $\mathrm{R}$ valley $\left(44^{\circ}\right.$ $05^{\prime} \mathrm{N}, 1^{\circ} 35^{\prime} \mathrm{E}$ ). Coll and subm 1978 by A Tavoso, Univ Marseille.

\section{Ly-2316. Tuf des cascades}

$$
24,000 \pm 500
$$

\section{Ly-2315. Tuf des Roches du Château}

$32,500 \pm 1000$

General Comment: despite uncertainty of this material, both dates agree with geol interpretations of age formation of both tufas: one is attributed to Late Würmian, other to Würmian interstadial. Ly-2315 should be considered min age (Ambert \& Tavoso, 1981).

\section{E. Samples from marine and lagoonal sediments}

\section{Lac Tanma series, Cayar region, Sénégal}

Table 13 lists samples of gray or black clay with vegetal remains from several levels in borings from sediments of Tanma coastal lake $\left(14^{\circ} 54^{\prime} \mathrm{N}\right.$, $\left.17^{\circ} 05^{\prime} \mathrm{W}\right)$. Coll and subm 1979 by J Médus, Lab Bot Hist, Univ Marseille.

TABLE 13

Lac Tanma

\begin{tabular}{rcccc}
\hline $\begin{array}{c}\text { Sample } \\
\text { no. }\end{array}$ & Boring & Depth & $\begin{array}{c}\text { Dilution } \\
\text { ratio }\end{array}$ & Age BP \\
\hline Ly-2264 & S 4 & $7 \mathrm{~m}$ & $1 / 3$ & $7610 \pm 260$ \\
-1911 & $\mathrm{~S} 4$ & $8 \mathrm{~m}$ & $3 / 20$ & $5990 \pm 530$ \\
-2265 & $\mathrm{~S} 4$ & $12 \mathrm{~m}$ & $11 / 15$ & $7790 \pm 190$ \\
-2057 & $\mathrm{~S} 4$ & $15 \mathrm{~m}$ & 1 & $7790 \pm 150$ \\
-2058 & $\mathrm{~S} 4$ & $17 \mathrm{~m}$ & $1 / 7$ & $9550 \pm 480$ \\
-1893 & $\mathrm{~S} 4$ & $20 \mathrm{~m}$ & $1 / 5$ & $10,640 \pm 600$ \\
-1973 & $\mathrm{~S} 4 ?$ & $45 \mathrm{~m}$ & $5 / 6$ & $4070 \pm 250$ \\
-2023 & $\mathrm{~S} 4 ?$ & $48 \mathrm{~m}$ & $1 / 10$ & $6080 \pm 450$ \\
-2024 & $\mathrm{~S} 4 ?$ & $49 \mathrm{~m}$ & $3 / 20$ & $7170 \pm 400$ \\
-2266 & $\mathrm{~S} 2$ & $12 \mathrm{~m}$ & $13 / 30$ & $7760 \pm 240$ \\
-2267 & $\mathrm{~S} 2$ & $19 \mathrm{~m}$ & $7 / 30$ & $7830 \pm 260$ \\
-2268 & $\mathrm{~F} 5$ & $2 \mathrm{~m}$ & $1 / 6$ & $1560 \pm 240$ \\
-2269 & F 5 & $12 \mathrm{~m}$ & $14 / 15$ & $7510 \pm 150$ \\
\hline
\end{tabular}

General Comment (JM): Ly-1893, -1973, and -2029 remain unexplained. For all other samples, dates fit well with depths and sea-level fluctuation curve drawn from other data (Faure \& Elouard, 1967), mainly from Mauritania coast (Einsele et al, 1977). Ly-2058 and -1893 correspond with short transgression which rose again ca $8000 \mathrm{BP}$ (6 results) and reached max with Ly-1911. Ly-2268 indicates that Tanma Lake was lagoon up to recent period, like previously dated Retba Lake (R, 1976, v 18, p 68).

\section{Delta du Sénégal series, Sénégal}

Table 14 lists samples of marine shells from several geol secs of sediments of Sénégal R delta near Saint-Louis. Coll and subm 1978 to 1980 by J Monteillet, Dept Geol, Inst Fondamental Afrique Noire, Dakar. 
TABLE 14

Delta du Sénégal

\begin{tabular}{|c|c|c|c|c|c|}
\hline $\begin{array}{l}\text { Sample } \\
\text { no. }\end{array}$ & Site ref & Loc & Sample & $\begin{array}{l}\text { Dilution } \\
\text { ratio }\end{array}$ & Age BP \\
\hline Ly-2158 & Gandon (I-A bis) & $\left(16^{\circ} 56^{\prime} \mathrm{N}, 16^{\circ} 26^{\prime} \mathrm{W}\right)$ & Anadara senilis & 1 & $5200 \pm 120$ \\
\hline-2039 & $\begin{array}{l}\text { Piste Dahra-Linguère } \\
(8021)\end{array}$ & & $\begin{array}{l}\text { Limnicolaria } \\
\text { chudeanei }\end{array}$ & $1 / 2$ & $660 \pm 150$ \\
\hline-1931 & Ndig (2a) & $\left(16^{\circ} 17^{\prime} \mathrm{N}, 16^{\circ} 19^{\prime} \mathrm{W}\right)$ & $\begin{array}{l}\text { Pachymelania } \\
\text { aurita }\end{array}$ & $1 / 2$ & $1080 \pm 210$ \\
\hline-2045 & Mbodiène (8027) & $\left(16^{\circ} 12^{\prime} \mathrm{N}, 16^{\circ} 15^{\prime} \mathrm{W}\right)$ & $\begin{array}{l}\text { Pachymalania } \\
\text { aurita }\end{array}$ & 1 & $1440 \pm 120$ \\
\hline-2042 & Boubene I (8024) & $\left(16^{\circ} 07^{\prime} \mathrm{N}, 16^{\circ} 23^{\prime} \mathrm{W}\right)$ & $\begin{array}{l}\text { Pachymelania } \\
\text { aurita }\end{array}$ & $3 / 5$ & $1490 \pm 180$ \\
\hline-2043 & Djeus Boubene (8025) & $\left(16^{\circ} 08^{\prime} \mathrm{N}, 16^{\circ} 23^{\prime} \mathrm{W}\right)$ & $\begin{array}{l}\text { Pachymelania } \\
\quad \text { aurita }\end{array}$ & $1 / 2$ & $1580 \pm 160$ \\
\hline-1928 & Guembeul (K G 2) & $\left(15^{\circ} 55^{\prime} \mathrm{N}, 16^{\circ} 28^{\prime} \mathrm{W}\right)$ & Anadara senilis & 1 & $1290 \pm 130$ \\
\hline-1927 & Guembeul (K G l) & $\left(15^{\circ} 55^{\prime} \mathrm{N}, 16^{\circ} 28^{\prime} \mathrm{W}\right)$ & Anadara senilis & 1 & $1530 \pm 130$ \\
\hline-1926 & Khant (KTM 2,a) & $\left(16^{\circ} 02^{\prime} \mathrm{N}, 16^{\circ} 22^{\prime} \mathrm{W}\right)$ & $\begin{array}{l}\text { Pachymelania } \\
\text { aurita }\end{array}$ & $4 / 15$ & $1650 \pm 280$ \\
\hline-1925 & Khant (KTM 1b) & $\left(16^{\circ} 03^{\prime} \mathrm{N}, 16^{\circ} 21^{\prime} \mathrm{W}\right)$ & Anadara senilis & 1 & $2760 \pm 120$ \\
\hline-2041 & Dialame (8023) & $\left(16^{\circ} 08^{\prime} \mathrm{N}, 16^{\circ} 20^{\prime} \mathrm{W}\right)$ & $\begin{array}{l}\text { Pachymelania } \\
\text { aurita }\end{array}$ & $2 / 3$ & $3280 \pm 150$ \\
\hline-2044 & Saroigne 3 (8026) & $\left(16^{\circ} 12^{\prime} \mathrm{N}, 16^{\circ} 17^{\prime} \mathrm{W}\right)$ & $\begin{array}{l}\text { Pachymelania } \\
\text { aurita }\end{array}$ & $4 / 5$ & $3230 \pm 170$ \\
\hline-1932 & Savoigne (SV-Ic) & $\left(16^{\circ} 12^{\prime} \mathrm{N}, 16^{\circ} 18^{\prime} \mathrm{W}\right)$ & Anadara senilis & $14 / 15$ & $5310 \pm 240$ \\
\hline-1933 & Savoigne (SV-4b) & $\left(16^{\circ} 13^{\prime} \mathrm{N}, 16^{\circ} 17^{\prime} \mathrm{W}\right)$ & Anadara senilis & 1 & $5640 \pm 190$ \\
\hline-2040 & $\begin{array}{l}\text { Makhana puits 1)1 } 2 \\
(8022)\end{array}$ & $\left(16^{\circ} 05^{\prime} \mathrm{N}, 16^{\circ} 23^{\prime} \mathrm{W}\right)$ & Anadara senilis & 1 & $5770 \pm 130$ \\
\hline-1930 & Dialam Dia & $\left(16^{\circ} 08^{\prime} \mathrm{N}, 16^{\circ} 20^{\prime} \mathrm{W}\right)$ & Tourbe & 1 & $6060 \pm 150$ \\
\hline-1929 & Djeus boubene (I)j. B C) & $\left(16^{\circ} 08^{\prime} \mathrm{N}, 16^{\circ} 23^{\prime} \mathrm{W}\right)$ & Anridro senilis & 1 & $6080 \pm 190$ \\
\hline-1918 & Niaodoum $(S)$ & $\left(16^{\circ} 03^{\prime} \mathrm{N}, 16^{\circ} 24^{\prime} \mathrm{W}\right)^{\prime}$ & $\begin{array}{l}\text { Pachymelania } \\
\text { auvita }\end{array}$ & 1 & $2150 \pm 130$ \\
\hline-1919 & Ndiael (1) & $\left(16^{\circ} 17^{\prime} \mathrm{N}, 16^{\circ} 01^{\prime} \mathrm{W}\right)$ & $\begin{array}{l}\text { Typanotonus } \\
\text { fuscatus }\end{array}$ & 1 & $4450 \pm 140$ \\
\hline-1923 & Tieng-To $(10-11 \mathrm{~m})$ & $\left(16^{\circ} 18^{\prime} \mathrm{N}, 16^{\circ} 21^{\prime} \mathrm{W}\right)$ & Anadara senilis & 1 & $6980 \pm 190$ \\
\hline-1920 & Diama Do $(2-3 \mathrm{~m})$ & & Pachymelania $s p$ & $3 / 10$ & $1690 \pm 220$ \\
\hline-1924 & Tieng T $5(21-22 \mathrm{~m})$ & $\left(16^{\circ} 18^{\prime} \mathrm{N}, 16^{\circ} 21^{\prime} \mathrm{W}\right)$ & $\begin{array}{l}\text { Pachymelania } \\
\text { tympanotonus }\end{array}$ & $1 / 10$ & $7320 \pm 600$ \\
\hline-1922 & Diama Do $(10 \mathrm{~m})$ & $\left(16^{\circ} 11^{\prime} \mathrm{N}, 16^{\circ} 25^{\prime} \mathrm{W}\right)$ & Anadara senilis & 1 & $6990 \pm 180$ \\
\hline-1921 & Diama Do $(11.5$ à $12 \mathrm{~m})$ & $\left(16^{\circ} 11^{\prime} \mathrm{N}, 16^{\circ} 25^{\prime} \mathrm{W}\right)$ & Anadara senilis & 1 & $\geqslant 33,700$ \\
\hline
\end{tabular}

General Comment (JM): Ly-2158 confirms previously pub result, Ly-1346: $5200 \pm 210(\mathrm{R}, 1979, \mathrm{v} 21, \mathrm{p} 426)$. These two series establish local variation curve of sea level from $8000 \mathrm{BP}$ to present and amount of continental flexure during last transgression (Faure et al, 1980). They also indicate wet climatic phase between 2000 and $3000 \mathrm{BP}$.

\section{Salinas series, Alicante prov, Spain}

Clay with very low organic content from two depths in upper sediments of shallow pond at Salinas near Elda $\left(38^{\circ} 27^{\prime} \mathrm{N}, 0^{\circ} 57^{\prime} \mathrm{W}\right)$. Coll and subm 1978 by G Truc, Dept Geol, Univ Lyon. (4/15 diluted samples)

\section{Ly-1654. 25cm$$
1850 \pm 400
$$ \\ Ly-1653. $45 \mathrm{~cm}$ \\ $1510 \pm 390$}

General Comment (GT): small amount of organic matter prevents distinction of two layers only separated by $20 \mathrm{~cm}$ depth. Average value sug- 
gests relatively high sedimentation rate of ca $20 \mathrm{~cm}$ millennium for bottom sediments of basin which was formed by Triassic diapir and is still salt marsh.

\section{Mas de Listel series, Le Grau du Roi, Gard}

Marine shells from present surface of Listel-Ventadis, ancient offshore bar in SW part of Rhône R delta $\left(43^{\circ} 45^{\prime} \mathrm{N}, 4^{\circ} 10^{\prime} \mathrm{E}\right)$. Coll and subm 1978 by J Archambault, Univ Orsay and A L'Homer, Bur Recherche Geol Min, Orléans, during study of offshore bar formations of Rhône delta.

\section{Ly-1764. Coupe de Mondragon, J9}

$1090 \pm 200$

Tests of Cerastoderma glaucum, shells.

Ly-1765. Cordon de Listel-Ventadis, no. 21

$1880 \pm 230$

Several spp of Cardiaces shells. (9/10 diluted sample)

General Comment (JA): both dates give Holocene age to Listel-Ventadis offshore bar, in agreement with general trend of coastal evolution deduced from other data (see, eg, Le Grau du Roi series, R, 1979, v 21, p 426; Bazile et al, 1981); this may be due to different chemical evolution of shells (Archambault-Guézou, in press).

Ly-2035. L'Aubette, Berre l'étang, Bouches du Rhône $1740 \pm 430$

Shells (Chlamys glabra) from remains of quarry $\left(43^{\circ} 27^{\prime} \mathrm{N}, 5^{\circ} 10^{\prime} \mathrm{E}\right)$. Coll 1980 by E Colomb, Univ Marseille, and subm 1980 by A Prieur, Dept Geol, Univ Lyon. (1/5 diluted sample). Comment (AP): paleontol expected age, end of Quaternary. Recent age of shells suggest they did not come from geol terrace but were brought in by man.

\section{Ly-2105. Corail de Uré, Ile des Pins, New Caledonia $19,490 \pm 330$}

Calcium carbonate from cement of coral breccia found during dig-

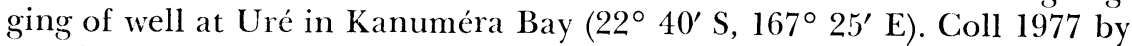
D Frimigacci and subm 1980 by F Poplin, Mus Natl Hist Nat, Paris. Coral breccia contains Sylviornis neocaledoniae fauna. Comment (FP): main coral level in which Uré well was dated to $>100,000 \mathrm{yr}$ by UTh dating method. ${ }^{14} \mathrm{C}$ date suggests coral breccia may assoc old coral and much younger material and cement but does not attribute age to fauna, as very low collagen content of bones prevents direct measurement of bones (Poplin, 1980).

\section{La Mer Pélagienne series, Tunisia}

Table 15 lists samples from borings, dredgings, and collns made 1976 and 1977 in deep sea, on continental plateau, and on continent in Pelagian Sea region (Gabès Gulf) during sedimentol study of continental platform of region by Co fr Pétroles and Soc Natle Elf-Aquitaine. 10\% of 760 samples were dated by Lyon, Gif-sur-Yvette, and Monaco ${ }^{14} \mathrm{C}$ labs. Details of environment, sample descriptions, interpretations of results were pub (Burollet, Clairefond, \& Winnock, 1979). Most measurements were made on total carbonate fraction of samples (except Ly-1753 and -1679) because 
some preliminary assays showed that results obtained on organic fraction and those on carbonate fraction (eg, Ly-1753/1752, Ly-1679/1680) are close enough for purposes of study (Delibrias \& Evin, 1979). As there was very little organic matter and almost no detrital carbonates, dates on total carbonate fraction was assumed sufficient.

TABLE 15

La Mer Pélagienne

\begin{tabular}{|c|c|c|c|c|c|c|c|}
\hline \multicolumn{8}{|c|}{ Gulf borings } \\
\hline $\begin{array}{c}\text { Lab } \\
\text { no. }\end{array}$ & $\begin{array}{c}\text { Sample } \\
\text { no. }\end{array}$ & Depth & $\begin{array}{c}\text { Colln } \\
\mathrm{yr}\end{array}$ & Loc & $\begin{array}{l}\text { Dilution } \\
\text { ratio }\end{array}$ & $\begin{array}{c}\delta^{13} \mathrm{C} \\
\% \%\end{array}$ & Age BP \\
\hline$y-1717$ & KST-10 & $20-30 \mathrm{~cm}$ & 1976 & $\left(33^{\circ} 52^{\prime} \mathrm{N}, 10^{\circ} 26^{\prime} \mathrm{E}\right)$ & 1 & +2.29 & $21,250 \pm 550$ \\
\hline-1726 & KST-19 & $150-162 \mathrm{~cm}$ & 1976 & $\left(31^{\circ} 14^{\prime} \mathrm{N}, 10^{\circ} 50^{\prime} \mathrm{E}\right)$ & $4 / 5$ & +2.29 & $8580 \pm 330$ \\
\hline-1727 & KST-19 & $325-327 \mathrm{~cm}$ & 1976 & $\left(31^{\circ} 14^{\prime} \mathrm{N}, 10^{\circ} 50^{\prime} \mathrm{E}\right)$ & 1 & +2.29 & $18,350 \pm 440$ \\
\hline-1728 & KST-19 & $445-455 \mathrm{~cm}$ & 1976 & $\left(31^{\circ} 14^{\prime} \mathrm{N}, 10^{\circ} 50^{\prime} \mathrm{E}\right)^{\prime}$ & 1 & +2.29 & $27,200 \pm 1000$ \\
\hline-1718 & $\mathrm{KST}-21$ & Surface & 1976 & $\left(31^{\circ} 17^{\prime} \mathrm{N}, 11^{\circ} 05^{\prime} \mathrm{E}\right)$ & 1 & +2.29 & $4630 \pm 160$ \\
\hline-1711 & $\mathrm{KST}-21$ & $220-230 \mathrm{~cm}$ & 1976 & $\left(31^{\circ} 17^{\prime} \mathrm{N}, 11^{\circ} 05^{\prime} \mathrm{E}\right)$ & 1 & +2.29 & $9930 \pm 210$ \\
\hline-1677 & KST-21 & $225-230 \mathrm{~cm}$ & 1976 & $\left(31^{\circ} 17^{\prime} \mathrm{N}, 11^{\circ} 05^{\prime} \mathrm{E}\right)$ & 1 & +2.29 & $9830 \pm 230$ \\
\hline-1678 & KST-21 & $435-440 \mathrm{~cm}$ & 1976 & $\left(31^{\circ} 17^{\prime} \mathrm{N}, 11^{\circ} 05^{\prime} \mathrm{E}\right)$ & 1 & +2.0 & $27,100 \pm 1000$ \\
\hline-1712 & KST-21 & $440-447 \mathrm{~cm}$ & 1976 & $\left(31^{\circ} 17^{\prime} \mathrm{N}, 11^{\circ} 05^{\prime} \mathrm{E}\right)$ & 1 & +1.5 & $31,200 \pm 2000$ \\
\hline-1713 & KST-102 & $221-238 \mathrm{~cm}$ & 1976 & $\left(34^{\circ} 19^{\prime} \mathrm{N}, 11^{\circ} 52^{\prime} \mathrm{E}\right)$ & $1 / 2$ & +3.6 & $12,600 \pm 500$ \\
\hline-1714 & KST-102 & $458-479 \mathrm{~cm}$ & 1976 & $\left(34^{\circ} 19^{\prime} \mathrm{N}, 11^{\circ} 52^{\prime} \mathrm{E}\right)$ & 1 & +2.1 & $20,740 \pm 550$ \\
\hline-1725 & KST-103 & $190-200 \mathrm{~cm}$ & 1976 & $\left(34^{\circ} 21^{\prime} \mathrm{N}, 12^{\circ} 07^{\prime} \mathrm{E}\right)$ & 1 & & $21,300 \pm 500$ \\
\hline-1679 & KST -104 & $58-63 \mathrm{~cm}$ & 1976 & $\left(34^{\circ} 20^{\prime} \mathrm{N}, 12^{\circ} 22^{\prime} \mathrm{E}\right)$ & $1 / 10$ & +9.90 & $11,200 \pm 860$ \\
\hline-1680 & KST-104 & $58-6.3 \mathrm{~cm}$ & 1976 & $\left(34^{\circ} 20^{\prime} \mathrm{N}, 12^{\circ} 22^{\prime} \mathrm{E}\right)$ & 1 & +2.90 & $12,960 \pm 260$ \\
\hline-1681 & KST-104 & $117-120 \mathrm{~cm}$ & 1976 & $\left(34^{\circ} 20^{\prime} \mathrm{N}, 12^{\circ} 22^{\prime} \mathrm{E}\right)$ & $1 / 2$ & +2.90 & $18,300 \pm 800$ \\
\hline .1719 & KST-106 & $10-20 \mathrm{~cm}$ & 1976 & $\left(34^{\circ} 20^{\prime} \mathrm{N}, 12^{\circ} 51^{\prime} \mathrm{E}\right)$ & $5 / 6$ & +1.6 & $13,300 \pm 350$ \\
\hline-1682 & KST-106 & $168-170 \mathrm{~cm}$ & 1976 & $\left(34^{\circ} 20^{\prime} \mathrm{N}, 12^{\circ} 51^{\prime} \mathbf{E}\right)$ & $9 / 10$ & -1.6 & $12,850 \pm 400$ \\
\hline-1683 & KST-106 & $170-172 \mathrm{~cm}$ & 1976 & $\left(34^{\circ} 20^{\prime} \mathrm{N}, 12^{\circ} 5 \mathrm{l}^{\prime} \mathbf{E}\right)$ & $2 / 3$ & -1.6 & $13,490 \pm 550$ \\
\hline-1684 & KST-106 & $325-335 \mathrm{~cm}$ & 1976 & $\left(34^{\circ} 20^{\prime} \mathrm{N}, 12^{\circ} 51^{\prime} \mathbf{E}\right)$ & 1 & +1.3 & $13,650 \pm 320$ \\
\hline-1685 & KST -107 & $32-36 \mathrm{~cm}$ & 1976 & $\left(34^{\circ} 27^{\prime} \mathrm{N}, 12^{\circ} 12^{\prime} \mathrm{E}\right)$ & 1 & +1.7 & $13,050 \pm 260$ \\
\hline-1686 & KST- 107 & $60-65 \mathrm{~cm}$ & 1976 & $\left(34^{\circ} 27^{\prime} \mathrm{N}, 12^{\circ} 12^{\prime} \mathrm{E}\right)$ & 1 & +1.7 & $17,200 \pm 450$ \\
\hline-1721 & KST -110 & $225-240 \mathrm{~cm}$ & 1976 & $\left(34^{\circ} 40^{\prime} \mathrm{N}, 13^{\circ} 15^{\prime} \mathrm{E}\right)$ & $2 / 3$ & +0.3 & $22,400 \pm 800$ \\
\hline-1720 & $\mathrm{KST}-110$ & $350-365 \mathrm{~cm}$ & 1976 & $\left(34^{\circ} 40^{\prime} \mathrm{N}, 13^{\circ} 15^{\prime} \mathrm{E}\right)$ & 1 & +0.1 & $23,300 \pm 750$ \\
\hline-1722 & $\mathrm{KST}-118$ & $445-458 \mathrm{~cm}$ & 1976 & $\left(34^{\circ} 46^{\prime} \mathrm{N}, 13^{\circ} 04^{\prime} \mathrm{E}\right)$ & 1 & +1.6 & $27,600 \pm 1000$ \\
\hline-1723 & C. -27 & $170-185 \mathrm{~cm}$ & 1977 & $\left(36^{\circ} 38^{\prime} \mathrm{N}, 12^{\circ} 18^{\prime} \mathrm{E}\right)$ & $5 / 6$ & -0.1 & $18,020 \pm 520$ \\
\hline-1724 & C. -27 & $560-575 \mathrm{~cm}$ & 1977 & $\left(36^{\circ} 38^{\prime} \mathrm{N}, 12^{\circ} 18^{\prime} \mathrm{E}\right)$ & $2 / 3$ & +0.4 & $20,100 \pm 800$ \\
\hline \multicolumn{8}{|c|}{ Dredging and "Doris" boring in Gulf } \\
\hline$y-1687$ & DRT-16 & $0 \mathrm{~cm}$ & 1976 & $\left(31^{\circ} 10^{\prime} \mathrm{N}, 10\right.$ & 1 & & $1500 \pm 140$ \\
\hline-1715 & DW -2 & $3245 \mathrm{~cm}$ & 1976 & $\left(34^{\circ} 26^{\prime} \mathrm{N}, 11^{\circ} 18^{\prime} \mathbf{E}\right)$ & 1 & & $30,500 \pm 1700$ \\
\hline-1716 & DW -2 & $4296 \mathrm{~cm}$ & 1976 & $\left(34^{\circ} 26^{\prime} \mathrm{N}, 11^{\circ} 18^{\prime} \mathrm{E}\right)$ & 1 & +1.4 & $\geqslant 35,000$ \\
\hline \multicolumn{8}{|c|}{ Sampling on continent and in Sebkhra region } \\
\hline Ly- 1757 & HA-1 & $45-50 \mathrm{~cm}$ & 1977 & $\left(34^{\circ} 21^{\prime} \mathrm{N}, 10^{\circ} 19^{\prime} \mathrm{E}\right)$ & $2 / 5$ & +2.4 & $5930 \pm 340$ \\
\hline-1707 & HA -40 & $0 \mathrm{~cm}$ & 1977 & $\left(34^{\circ} 21^{\prime} \mathrm{N}, 10^{\circ} 13^{\prime} \mathbf{E}\right)$ & $1 / 2$ & -1.5 & $8580 \pm 360$ \\
\hline-1708 & $\mathrm{KN}-41$ & $0 \mathrm{~cm}$ & 1977 & $\left(34^{\circ} 40^{\prime} \mathrm{N}, 11^{\circ} 08^{\prime} \mathbf{E}\right)$ & 1 & -4 & $34,500 \pm 2000$ \\
\hline-1705 & KN -54 & $0 \mathrm{~cm}$ & 1977 & $\left(34^{\circ} 37^{\prime} \mathrm{N}, 11^{\circ} 03^{\prime} \mathrm{E}\right)$ & 1 & & $9730 \pm 190$ \\
\hline-1706 & $\mathrm{KN} 60$ & $0 \mathrm{~cm}$ & 1977 & $\left(34^{\circ} 47^{\prime} \mathrm{N}, 11^{\circ} 16^{\prime} \mathrm{E}\right)$ & 1 & & $17,760 \pm 480$ \\
\hline-2006 & KSS & & 1977 & $\left(34^{\circ} 19^{\prime} \mathrm{N}, 10^{\circ} 18^{\prime} \mathrm{E}\right)$ & 1 & & $5140 \pm 180$ \\
\hline \multicolumn{8}{|c|}{ Dredging in herbariums region } \\
\hline Ly-1709 & $\mathrm{KK} \cdot 7$ & $20 \mathrm{~cm}$ & 1977 & $\left(34^{\circ} 42^{\prime} \mathrm{N}, 11^{\circ} 19^{\prime} \mathrm{E}\right)$ & $1 / 2$ & +2.6 & $4420 \pm 300$ \\
\hline-1751 & KK-24 & $120-130 \mathrm{~cm}$ & 1977 & $\left(34^{\circ} 40^{\prime} \mathrm{N}, 11^{\circ} 10^{\prime} \mathrm{E}\right)$ & 1 & +3.1 & $1830 \pm 140$ \\
\hline-1752 & KK -29 & $90-100 \mathrm{~cm}$ & 1977 & $\left(34^{\circ} 44^{\prime} \mathrm{N}, 11^{\circ} 19^{\prime} \mathrm{E}\right)$ & 1 & +2.3 & $2430 \pm 160$ \\
\hline-1753 & KK -29 & $90-100 \mathrm{~cm}$ & 1977 & $\left(34^{\circ} 44^{\prime} \mathrm{N}, 11^{\circ} 19^{\prime} \mathrm{E}\right)$ & $9 / 10$ & & $1040 \pm 250$ \\
\hline-1754 & KK -29 & $100-110 \mathrm{~cm}$ & 1977 & $\left(34^{\circ} 45^{\prime} \mathrm{N}, 11^{\circ} 19^{\prime} \mathbf{E}\right)$ & 1 & +4.1 & $1860 \pm 150$ \\
\hline-1710 & K K -44 & $38 \mathrm{~cm}$ & 1977 & $\left(34^{\circ} 48^{\prime} \mathrm{N}, 11^{\circ} 20^{\prime} \mathbf{E}\right)$ & $5 / 6$ & +2.2 & $5920 \pm 260$ \\
\hline-1755 & KK -45 & $130-140 \mathrm{~cm}$ & 1977 & $\left(34^{\circ} 49^{\prime} \mathrm{N}, 11^{\circ} 18^{\prime} \mathrm{E}\right)$ & 1 & & $3450 \pm 150$ \\
\hline-1756 & KK -46 & $125-135 \mathrm{~cm}$ & 1977 & $\left(34^{\circ} 49^{\prime} \mathrm{N}, 11^{\circ} 17^{\prime} \mathrm{E}\right)$ & 1 & +4.7 & $1150 \pm 130$ \\
\hline
\end{tabular}


General Comment: 24 and 9 other results, respectively, were obtained by Gif and Monaco labs from same zone or same cores. All results agree perfectly with each other and all values were pub by CFP Soc and T Lajmi, Geol Survey Tunisia (Burollet, Clairefond, \& Winnock, 1979). Conclusions drawn from ${ }^{14} \mathrm{C}$ analyses are described in Delibrias \& Evin (1979). All results agree with data from other facets of study (palynol, sedimentol, and paleontol), demonstrating that carbonate sediments of Pelagian Sea were recently deposited either during Neotyrrhenian (Late Würmian) period or Versillian (Holocene) period.

\section{Ly-2420. Oued Akarit, Tunisia}

$8240 \pm 170$

Cardium shells from $+10 \mathrm{~m}$ alt in lagoonal layer embedded in Würmian terrace of Oued Akarit R, SE of Gabès $\left(34^{\circ} 07^{\prime} \mathrm{N}, 7^{\circ} 40^{\prime} \mathrm{E}\right)$. Coll and subm 1981 by $\mathrm{P}$ Sanlaville, Univ Lyon. Comment (PS): date confirms other unpub measurements and suggests rise of shore of ca $20 \mathrm{~m}$ in eight millennia.

\section{Oued Karrouba series, Tunisia}

Marine shells from offshore bars between mouths of Oued Ferd and Oued Saquiet el Karrouba R, SE of Gabès (33 $47^{\prime} \mathrm{N}, 7^{\circ} 54^{\prime} \mathrm{E}$ ). Coll 1981 by R Paskoff and P Sanlaville and subm 1981 by P Sanlaville, Univ Lyon.

$$
\begin{array}{lll}
\text { Ly-2418. } & +3 \mathrm{~m} & \mathbf{5 5 3 0} \pm 160 \\
\text { Ly-2419. } & +4 \mathrm{~m} & \mathbf{5 4 9 0} \pm 130
\end{array}
$$

General Comment (PS): both values confirm assumed Holocene age of offshore bars which redeposit materials from ancient Thyrennian offshore bars. They also confirm two unpub results from neighboring Oued Melah $\mathrm{R}$ site, MC-2155: 6420 \pm 100; MC-2154: $6200 \pm 100$. These four dates indicate Holocene shore was higher than present sea levels in $S$ Tunisia.

\section{Tin Oueich series, Mauritania}

Marine shells from two calcareous beds outcropping at Tin Oueich, $25 \mathrm{~km} \mathrm{SE}$ of Nouackchott $\left(18^{\circ} 4^{\prime} \mathrm{N}, 15^{\circ} 49^{\prime} \mathrm{W}\right)$. Coll 1980 by J Evin et $\mathrm{D}$ Carité, Fr Tech assistance at Nouackchott (Carité, 1977).

\section{Ly-2160. Plateau}

Shells (Anadar senilis) from lumachelle layer at $+4 \mathrm{~m}$.

Ly-2189. Zone base

$29,900 \pm 600$

Shells (Crassostrea gasar) from falun layer at $+2 \mathrm{~m}$.

General Comment (DC): both layers are made of sediments deposited during transgressive phases. According to sediment facies, layer of lowest region of site was presumed to be deposited during Nouackchottian transgression which was dated many times (see, eg, Nouackchott series with Ly-350: $5510 \pm 120, \mathrm{R}, 1975$, v 17, p 15). However, both results indicate two phases of single transgression in site; Inchirian transgression which 
was also dated many times in region, eg, at Tafarit Cap, Ly-443: 31,400 +2300
-1800 $(\mathrm{R}, 1975, \mathrm{v} 17, \mathrm{p} 16)$.

\section{ARCHAEOLOGIC SAMPLES}

\section{A. Historic period}

Ly-2274. Pirogue du Lac de Paladru, Lepin, Isère $\quad \mathbf{5 8 0} \pm 230$

Wood from monoxyl barge found in mud of Paladru Lake $\left(45^{\circ} 27^{\prime} \mathrm{N}\right.$, $5^{\circ} 33^{\prime}$ E). Coll 1979 and subm 1980 by M Colardelle, Centre Archéol Hist, Grenoble. (2/5 diluted sample). Comment (MC): despite large uncertainty due to small sample size, date confirms historic period expected from iron nails fixed in wood (Laurent, 1968).

\section{Ly-2252. Garnat sur Engièvre, Allier $\quad 900 \pm 110$}

Wood from monoxyl barge from $2.5 \mathrm{~m}$ depth in alluvium of channel of Loire R $\left(46^{\circ} 38^{\prime} \mathrm{N}, 3^{\circ} 42^{\prime} \mathrm{E}\right)$. Coll and subm 1980 by M Sauget, Dir Antiquités Hist, Clermont-Ferrand. No assoc industry (Vertet, 1981). (9/10 diluted sample). Comment (JMS): date assigns medieval age to boat.

\section{Ly-2199. Epervans, Saône 1}

$1260 \pm 140$

Fragments of monoxyl barge found at Epervans, Saône et Loire $\left(46^{\circ}\right.$ $45^{\prime} \mathrm{N}, 4^{\circ} 55^{\prime} \mathrm{E}$ ). Coll and subm 1979 by $\mathrm{L}$ Bonnamour, Mus Denon, Châlon. Assoc mainly with Gallo-Roman ceramics but also with some Merovingian vases. Comment (LB): younger than expected, but date is not surprising for such a boat, shape of which remains fairly unchanged from Early Neolithic up to 19 th century AD.

\section{Ly-1845. La Tour des Chiens, Corenc, Isère}

$490 \pm 160$

Bark (Picea sp) found in mortar of stone wall $\left(45^{\circ} 14^{\prime} \mathrm{N}, 5^{\circ} 47^{\prime} \mathrm{E}\right)$. Coll and subm 1978 by M Lafont, Corenc. Comment (ML): text certifies that "La Tour des Chiens" was already built in AD 1241; date indicates either wall was built after main bldg or was later repaired.

\section{Bois de l'Abbaye de St Victor series, Marseille, Bouches du Rhône}

Fragments of two pieces of wood from treasure of Saint Victor abbey $\left(48^{\circ} 18^{\prime} \mathrm{N}, 5^{\circ} 23^{\prime} \mathrm{E}\right)$. Coll and subm Dec 1979 by A Pons, Lab Palynol, Marseille and measured in March 1980. According to old tradition, wood was considered relics from lst century AD; they were brought to Marseille in 13th century, but disappeared for short time during French Revolution and beginning of 19th century. Three dates are possible: beginning of Christian era, Middle ages, or 19th century.

$$
\begin{array}{lll}
\text { Ly-1990. } & \text { Bois long } & 710 \pm 150 \\
\text { Ly-1991. } & \text { Bois court } & 750 \pm 150
\end{array}
$$

General Comment (AP): closeness of dates of both wood fragments which also belong to same sp (Salix alba L) confirms they are of same origin if not same tree. Dates are of Middle ages probably corresponding to Crusades. They also indicate that temporary disappearance of wood did not affect their relative authenticity. 


\section{Eglise de Viuz series, Faverges, Haute Savoie}

Human bones and charcoal (Ly-1879) from grave in Saint-JeanBaptiste Church $\left(45^{\circ} 45^{\prime} \mathrm{N}, 6^{\circ} 17^{\prime} \mathrm{E}\right)$. Coll 1978 and subm 1979 by $\mathrm{M}$ Colardelle.

$$
\begin{array}{ll}
\text { Ly-1877. } & 150 \\
\text { Ly-1878. } & 71 \\
\text { Ly-1879. } & 61 \\
\text { Ly-1880. } & 29
\end{array}
$$$$
490 \pm 120
$$

$$
1010 \pm 130
$$$$
2210 \pm 130
$$$$
880 \pm 140
$$

General Comment (MC): Ly-1879 may be too old because of vicinity of older archaeol layers. Three other values seem to confirm archaeol data that nobody was buried in church after 15 th or 16th century (Colardelle, 1980).

\section{Nécropole Saint-Girard series, Sainte-Croix, Drôme}

Bones and charcoal (Ly-1874) from several graves in Saint-Girard necropolis $\left(44^{\circ} 46^{\prime} \mathrm{N}, 5^{\circ} 16^{\prime} \mathrm{E}\right)$. Coll 1978 and subm 1979 by M Colardelle.

Ly-1871. Sépulture 29

$$
\begin{aligned}
870 & \pm 150 \\
1010 & \pm 140 \\
730 & \pm 130
\end{aligned}
$$

Ly-1872. Sépulture 55

Ly-1873. Sépulture à chambre

General Comment (MC): three dates establish chronology of 1st occupation period of necropolis.

\section{Ly-2293. Les Bellets, Saint-Pancrasse, Isère}

$1610 \pm 130$

Charcoal from presumed lime-kiln excavation on Les Petites-Roches Plateau $\left(45^{\circ} 16^{\prime} \mathrm{N}, 5^{\circ} 53^{\prime} \mathrm{E}\right)$. Coll 1978 by C Jail and subm 1979 by $\mathrm{M}$ Colardelle, Centre archéol Hist, Grenoble. Plateau was occupied since High Middle age. (5/6 diluted sample). Comment (MC): date is a little older than expected and suggests that lime kilns were occupied as soon as end of Roman times.

\section{Ly-2306. Le Pusmin de Saint Armel, Sarzeau, Morbihan}

Wood fragment from lintel of door of house $\left(47^{\circ} 31^{\prime} \mathrm{N}, 2^{\circ} 48^{\prime} \mathrm{W}\right)$. Coll and subm 1980 by P Gevin, Geol Dept, Univ Lyon. Comment (PG): previous date on timber of basement of house was much older: Ly-1626: $1250 \pm 150(\mathrm{R}, 1979, \mathrm{v} 21, \mathrm{p} 428)$. Present date, ca AD 1570 , exactly fits with inscription on another lintel of house.

\section{Ly-2179. Sépulture 2, CD 258, Saint-Germain-des Fossés, Allier}

$1130 \pm 120$

Human bones from Sépulture 2 from graves at side of CD $258 \mathrm{rd}\left(46^{\circ}\right.$ 12' N, 326 $6^{\prime}$ E). Coll 1979 and subm 1980 by J P Daugas and L Magoga, Dir Antiquités Préhist Auvergne, Clermont-Ferrand, and J P Raynal, Inst Quaternaire Bordeaux, Talence. (2/5 diluted sample). Comment 
(JPD \& JPR): date confirms High Middle age sepulture close to GalloRoman archaeol site overlapping child's grave probably from Neolithic. period.

Ly-1777. Mérignac, Gironde

Bones from grave lying at level $-1.6 \mathrm{~m}$ in SE apse of old church of Saint-Vincent $\left(44^{\circ} 5 \mathrm{l}^{\prime} \mathrm{N}, 0^{\circ} 39^{\prime} \mathrm{W}\right)$. Coll and subm 1977 by J Sautreau, Léognan. This lst Christian church at Mérignac was built on ruins of Roman bldg and became necropolis at beginning of Middle ages. Comment (JS): date confirms archaeol hypothesis that grave cannot belong to end of Roman times.

\section{Brandes series, l'Alpes d'Huez, Isère}

Bones from cemetery of ancient village of Brandes $\left(45^{\circ} 05^{\prime} \mathrm{N}, 6^{\circ} 05^{\prime}\right.$ E). Coll 1978 by J Bruno and M C Bailly-Maître and subm 1979 by $M$ Colardelle. Village was settled during 11 th century near silver-lead mine and deserted during 15 th century.

Ly-2272. Tombe NE

$600 \pm 120$

From NE grave, at $2.5 \mathrm{~m}$ depth. (4/5 diluted sample)

Ly-2273. Tombe NC

$610 \pm 150$

From NC grave at $2.5 \mathrm{~m}$ depth. (7/30 diluted sample)

General Comment (MC): both dates are mid-14th century and in expected range, confirming contemporaneity of graves.

\section{Ly-1874. Eglise Saint-Martin, Saint-Julien-en-}

Genèvois, Haute Savoie

$1080 \pm 140$

Charcoal from grave in Saint-Martin Funerary basilica $\left(46^{\circ} 08^{\prime} \mathrm{N}, 6^{\circ}\right.$ 05' E). Coll 1978 and subm 1980 by M Colardelle. Comment (MC): date confirms fairly late use of basilica (Colardelle, 1980).

\section{Roissard series, Isère}

Charcoal from dwelling (Ly-1875) and necropolis $\left(44^{\circ} 53^{\prime} \mathrm{N}, 5^{\circ} 38^{\prime}\right.$ E). Coll 1978 and subm 1979 by M Colardelle.

\section{Ly-1875. Fond de cabane \\ $1180 \pm 130$}

From hearth in cabin of presumed Merovingian dwelling.

\section{Ly-1876. Sépulture 9}

$1640 \pm 140$

From Sépulture 9 of necropolis containing artifacts of High Middle ages. (1/2 diluted sample)

General Comment (MC): both dates agree with expected ages; Ly-1875 dates ca $\mathrm{AD} 770$, very end of Merovingian times, and Ly-1876, ca AD 310, beginning of High Middle ages, considering uncertainty margin; necropolis must have been in use early.

\section{Ly-1801. Le Champ des Pics, Saint-Yvoine, Puy de \\ Dôme}

$1420 \pm 200$

Human bones from Le Champ des Pics Cemetery $\left(45^{\circ} 35^{\prime} \mathrm{N}, 3^{\circ} 13^{\prime}\right.$

E). Coll 1880 by M Millon and subm by A Cogoluehnes, Dept Geol, Univ 
Lyon. Comment (AC): date indicates Middle age for tombs without assoc industry.

Les Valleyres series, Cussac-sur-Loire, Haute Loire

Samples from foot-hill sediments underlying rocks $\left(44^{\circ} 58^{\prime} \mathrm{N}, 3^{\circ} 55^{\prime}\right.$

E). Coll 1979 and subm 1981 by A Crémillieux, Le Monastier-sur-Gazeille, Haute-Loire. Assoc with less characteristic industry and rich fauna (Crémillieux, 1979).

Ly-2437. 1

$1750 \pm 160$

Charcoal. (23/30 diluted sample)

Ly-2439. 2

$1880 \pm 220$

Bones. (4/15 diluted sample)

General Comment (AC): both dates are much younger than expected. Age of ca 30,000 yr was expected from regular stratification of sediment and presence of cut flints. Modern value cannot be explained without complete study of site.

Ly-2344. Font Carluze, Perols/Vézère, Corrèze

$2020 \pm 110$

Wood fragment from Gallo-Roman oak pipe from peat bog $\left(45^{\circ} 35^{\prime}\right.$ N, 2 ${ }^{\circ} 02^{\prime}$ W). Coll 1969 and subm 1980 by G Lintz, Dir Antiquités Hist, Limoges. Comment (GL): dated to calibrate lst dendrochronol curve in Limousin region. Date confirms expected Gallo-Roman period.

\section{B. Protohistoric period}

\section{Kandiama series, Velingara, Haute Casamance, Sénégal}

Table 16 lists samples of charcoal from fill of three galleries at a few $\mathrm{m}$ depth in soil $\left(13^{\circ} 10^{\prime} \mathrm{N}, 13^{\circ} 51^{\prime} \mathrm{W}\right)$. Coll and subm 1979 by J Girard, Lab Ethnol, Univ Lyon II. Galleries are assumed troglodyte habitats or underground hiding places of ancient kingdom of Tekrour, destroyed ca AD 1350; they also might be drifts of ancient laterite mine.

TABLE 16

Kandiama

\begin{tabular}{rlccc}
\hline $\begin{array}{c}\text { Sample } \\
\text { no. }\end{array}$ & Gallery & $\begin{array}{c}\text { Dilution } \\
\text { ratio }\end{array}$ & $\delta^{13} \mathrm{C} \%$ o & Conventional age \\
\hline Ly-1993 & Kandamia C'h & 1 & $-0.1 \pm 1.7$ & Modern \\
-1992 & Kandamia C'm & 1 & $+0.7 \pm 1.8$ & Modern \\
-1994 & Kandamia C'b & 1 & $-0.2 \pm 1.6$ & Modern \\
-1995 & Kandamia C'b & $2 / 3$ & $+0.9 \pm 2.2$ & Modern \\
-1996 & Kandamia C'b & 1 & $0.00 \pm 1.8$ & Modern \\
-1997 & Kandamia C'b & 1 & $+2.4 \pm 1.9$ & Modern \\
\hline
\end{tabular}

General Comment: if charcoal was actually embedded in sediments, fill of galleries is modern. Dates do not confirm expected age (6th-7th century) attributed to all galleries. Modern values suggest that previous pub results (Kandiama series, R, 1979, v 21, p 431) are either too old or are apparent age of burned wood. Thus, both series cannot be used to confirm ethnol hypothesis on origin of galleries (Girard, 1980). 
Ly-2188. Mbaouane, Cayar, Sénégal

$1410 \pm 140$

Charcoal scattered in lowest levels of sandy dune $\left(14^{\circ} 44^{\prime} \mathrm{N}, 17^{\circ} 07^{\prime}\right.$ W) containing potsherds and overlying Neolithic site. Coll and subm 1980 by J Evin and A Ravisé, IFAN, Dakar. Comment (AR): date is much younger than expected and proves recent change in loc of dune with transport of relatively heavy material. Charcoal cannot be considered contemporaneous with site.

\section{Sintiou Bara series, Matam Dept, Sénégal}

Table 17 lists samples of charcoal from several levels in three archaeol excavations in ancient village Sintiou Bara, near Ourossogui, le Fleuve region $\left(15^{\circ} 42^{\prime} \mathrm{N}, 13^{\circ} 24^{\prime} \mathrm{W}\right)$. Coll 1977 and subm 1977 by G Thilmans, IFAN.

TABLE 17

Sintiou Bara

\begin{tabular}{rlccrr}
\hline Lab no. & $\begin{array}{c}\text { Sample } \\
\text { ref }\end{array}$ & $\begin{array}{c}\text { Excavation } \\
\text { sq }\end{array}$ & Depth & $\begin{array}{c}\text { Dilution } \\
\text { ratio }\end{array}$ & \multicolumn{1}{c}{ Age BP } \\
\hline Ly-1741 & IFAN 124 & M 16 & $205 \mathrm{~cm}$ & $2 / 5$ & $1470 \pm 210$ \\
-1742 & IFAN 126 & O 16 & $160 \mathrm{~cm}$ & $4 / 7$ & $970 \pm 150$ \\
-1743 & IFAN 127 & K 16 & $96 \mathrm{~cm}$ & $3 / 5$ & $1460 \pm 220$ \\
-1744 & IFAN 128 & K 17 & $134 \mathrm{~cm}$ & 1 & $1090 \pm 160$ \\
-1745 & IFAN 129 & K $16-17$ & $165 \mathrm{~cm}$ & 1 & $1550 \pm 140$ \\
\hline
\end{tabular}

General Comment (GT): results agree with archaeol data and other series from same type of site in region (Ogo, Saré Tioff, and Tioubalel series, below). With three unpub results: Dak-192: $900 \pm 110$ (sq L 14, 225cm), Dak-155: $1363 \pm 120$ (sq M 18, 270cm), and Gif-4522: $920 \pm 80$ (sq X 16, $255 \mathrm{~cm}$ ), series suggests site occupation for at least $600 \mathrm{yr}$ during $2 \mathrm{nd}$ half of 1st millennium AD and negates any relationship between depths and ages of layers.

\section{Saré Tioffi series, Podor Dept, Sénégal}

Samples from cut-off burial hillock in ancient village Saré Tioffi, le Fleuve region $\left(16^{\circ} 40^{\prime} \mathrm{N}, 14^{\circ} 58^{\prime} \mathrm{W}\right)$. Coll 1976 and subm by B Chavane, Dakar.

\section{Ly-2033. S-III $40 \mathrm{~cm}$}

Charcoal from geol level with grave; subm 1979.

\section{Ly-1937. S-III 95cm}

$$
1580 \pm 130
$$

Charcoal from layer overlying grave; subm 1978.

Ly-1603. S-III $80 \mathrm{~cm}$

$$
4830 \pm 770
$$
ple)

Bones of intact skeleton found in grave; subm 1976. (1/4 diluted sam-

General Comment (BC): 1st two measurements date site occupation and agree well with expected value and Ogo and Sintiou Bara series from same Iron age culture of ancient Tekrour Kingdom (Chavane, 1980). Ly-1603 is obviously too old for unknown reason even with widest statistical margin. 


\section{Ogo series, Matam Dept, Sénégal}

Samples from proto-historic Ogo village, Le Fleuve region $\left(15^{\circ} 34^{\prime} \mathrm{N}\right.$, $\left.13^{\circ} 17^{\prime} \mathrm{W}\right)$. Site is small hill with accumulation of cultural remains.

\section{Ly-2034. Charbons de bois $790 \pm 100$}

Charcoal from 60 to $70 \mathrm{~cm}$ depth in excavation sq $S_{2}$. Coll and subm 1979 by B Chavane. Assoc with iron metallurgy artifacts.

\section{Ly-2159. Torchis}

$1910 \pm 210$

Dried mud with much charred vegetal remains from wall of burned house at 50 to $70 \mathrm{~cm}$ depth in sq $\mathrm{S}_{2}$. Coll 1980 by J Evin to test use of sampling material (1/3 diluted sample) despite burning of $600 \mathrm{~g}$ of dried black mud.

General Comment (BC): Ly-2034 agrees with expected date corresponding with end of village occupation and ancient kingdom of Tekrour (Chavane, 1980). Two unpub dates were obtained for deeper layers in site: Gif-4529: $910 \pm 90$ (55cm depth) and Gif-4530: $1020 \pm 90$ (2.55m depth). Thus, three charcoal dates suggest at least 200-yr range of site. Ly-2159 is obviously too old, establishing that elements of mud wall cannot be used as sample. In fact, it seems that measured carbon partly comes from remaining organic matter in clay used for wall, not only from vegetal remains added to clay as temper.

\section{Tioubalel series, Matam Dept, Sénégal}

Charcoal from two depths in excavation at site of ancient village lying along Senegal R (16 $\left.{ }^{\circ} 16^{\prime} \mathrm{N}, 13^{\circ} 59^{\prime} \mathrm{W}\right)$. Coll 1977 by G Thilmans and subm 1980 by IFAN. Assoc with potsherds and copper and iron artifacts (Thilmans \& Ravisé, in press).

\section{Ly-2049. IFAN 148}

$1170 \pm 90$

From $53 \mathrm{~cm}$ depth; expected age: $600 \mathrm{BP}$.

\section{Ly-2048. IFAN 147}

$1960 \pm 400$

From $117 \mathrm{~cm}$ depth; expected age: 800 вр. (1/2 diluted sample)

General Comment (GT): both dates are older than expected but agree with those from other villages of same culture in Le Fleuve region (SaréTioff, Sintiou-Bara, and Ogo series, above).

\section{Fond-Brûlé series, Le Lorrain, Martinique}

Charcoal from level of lst Arawak period of Fond-Brûlé site $\left(10^{\circ} 30^{\prime}\right.$ N, $\left.61^{\circ} 00^{\prime} \mathrm{W}\right)$. Coll 1978 by M Mattioni and M Schvoerer and subm 1978 by M Schvoerer, Lab Physique Appl Archeol, Univ Bordeaux. Dated to cross-check dates by TL method. (1/6 diluted samples)

\section{Ly-2196. BDX-177, Carré K3 $1630 \pm 220$ \\ Ly-2197. BDX-175, Carré 04-P2

General Comment (MS): TL dates from same level are BDX-156: $2010 \pm$ $350 \mathrm{BP}$ and BDX-161: $1840 \pm 220 \mathrm{BP}$. Previous ${ }^{14} \mathrm{C}$ date for volcanic erup- 
tion which ended 1st occupation period of site ca 1655 BP. These five dates agree very well and disagree with two unpub dates from Nancy, ca 2360 and 2660 BP for same level. Another TL date was obtained for 2nd Arawak occupation period (assoc with Carribean industries): BDX-154: $1110 \pm 170 \mathrm{BP}$.

\section{Sou and Sou Blama Radjil series, Logone et Cahri, Cameroun}

Table 18 lists samples of charcoal from two open-air sites at Sou $\left(12^{\circ}\right.$ $\left.12^{\prime} \mathrm{N}, 14^{\circ} 42^{\prime} \mathrm{E}\right)$ and Sou Blama Radjil $\left(12^{\circ} 13^{\prime} \mathrm{N}, 14^{\circ} 42^{\prime} \mathrm{E}\right)$ near Afadé. Coll by J Rapp, Strasbourg and by J P Lebeuf, Paris. Assoc with industries of decorated ceramics and in some levels with metal, stone, or bone.

TABLE 18

Sou and Sou Blama Radjil

\begin{tabular}{rccccccc}
\hline $\begin{array}{c}\text { Lab } \\
\text { no. }\end{array}$ & Site & $\begin{array}{c}\text { Sample } \\
\text { no. }\end{array}$ & $\begin{array}{c}\text { Yr coll } \\
\text { and } \\
\text { subm }\end{array}$ & Layer & Depth & $\begin{array}{c}\text { Dilution } \\
\text { ratio }\end{array}$ & Age BP \\
\hline Ly-2002 & Sou (Pt XIX) & 41.1407 & 1979 & 3 & $100-110 \mathrm{~cm}$ & 1 & $500 \pm 130$ \\
-2003 & Sou Blama R & $168-277$ & 1979 & 3 & $240-247 \mathrm{~cm}$ & 1 & $2310 \pm 150$ \\
-2004 & Sou Blama R & $168-281$ & 1979 & 3 & $260-267 \mathrm{~cm}$ & 1 & $2280 \pm 170$ \\
-2005 & Sou Blama R & $168-296$ & 1979 & 3 & $300-307 \mathrm{~cm}$ & 1 & $2530 \pm 130$ \\
-2280 & Sou Blama R & $168-429$ & 1980 & $2 \mathrm{~b}$ & $148 \mathrm{~cm}$ & $2 / 15$ & $2570 \pm 240$ \\
-2281 & Sou Blama R & $168-519$ & 1980 & 4 & $304 \mathrm{~cm}$ & $1 / 5$ & $2740 \pm 210$ \\
-2282 & Sou Blama R & $168-539$ & 1980 & 5 & $340 \mathrm{~cm}$ & $1 / 5$ & $3200 \pm 250$ \\
-2283 & Sou Blama R & $168-561$ & 1980 & 7 & $405-407 \mathrm{~cm}$ & $7 / 30$ & $2430 \pm 250$ \\
-2284 & Sou Blama R & $168-563$ & 1980 & 7 & $430-440 \mathrm{~cm}$ & $1 / 6$ & $3280 \pm 360$ \\
\hline
\end{tabular}

General Comment (JR): only date from Sou site, Ly-2002, agrees with expected age corresponding to youngest Sao cultural phase, later than 10th century AD. Except Ly-2283, which is obviously too young, all dates of Sou Blama site are consistent with stratigraphy despite large statistical margins of samples coll in 1980. Dates also agree with unpub result, Gif-4821: 2340 \pm 100 for Layer 3 but agree with another unpub result: $2800 \pm 110$ for Layer 7. Dates indicate relatively long duration of earliest phase of Sao culture assoc with fine ceramics.

Ly-2104. Sanctuary Cybèle, Fourvière, Lyon, France $2100 \pm 140$

Charcoal from level rich in organic matter lying under SW angle of Cybèle sanctuary in Roman site $\left(44^{\circ} 46^{\prime} \mathrm{N}, 4^{\circ} 50^{\prime} \mathrm{E}\right)$. Coll 1978 and subm 1979 by A Audin, Gallo-Roman Mus Fourvière, Lyon. (3/5 diluted sample). Comment (AA): date agrees with expected age, ie, just before Roman epoch as dated layer underlies lst level of Roman bldg.

\section{Tureng-Tepe series, Gorgan, Iran}

Table 19 lists samples of charred wood from several fire levels indicating end of several occupation periods of Tureng Tepe tell $\left(36^{\circ} 55^{\prime} \mathrm{N}\right.$, $54^{\circ} 35^{\prime} \mathrm{E}$ ). Coll from 1969 to 1977 by J Deshayes and subm 1974 to 1978 by J Deshayes and S Cleuziou, Centre Recherche Archeol; Paris. 
TABLE 19

Tureng-Tepe

\begin{tabular}{|c|c|c|c|c|c|c|c|}
\hline $\begin{array}{l}\text { Lab } \\
\text { no. }\end{array}$ & & $\begin{array}{c}\text { Sample } \\
\text { no. }\end{array}$ & $\begin{array}{c}\text { Colln } \\
\mathrm{yr}\end{array}$ & $\begin{array}{l}\text { Level and } \\
\text { culture }\end{array}$ & $\begin{array}{c}\text { Cali- } \\
\text { brated } \\
\text { expected } \\
\text { age }\end{array}$ & $\begin{array}{l}\text { Dilution } \\
\text { ratio }\end{array}$ & Age BP \\
\hline Ly-1149 & $\mathbf{T T}$ & C-71-10 & 1971 & VII & AD 700 & 1 & $1410 \pm 140$ \\
\hline-2248 & $\mathbf{T} \mathbf{T}$ & $77-4$ & 1977 & $\begin{array}{l}\text { Soil of Sassanide } \\
\text { fortress }\end{array}$ & AD 500 & 1 & $1650 \pm 100$ \\
\hline-1065 & $\mathbf{T T}$ & C-71-9 & 1971 & Soil of fortress & AD 500 & 1 & $1940 \pm 80$ \\
\hline-2249 & TT & $71-5$ & 1977 & VI & AD 500 & $1 / 5$ & $3440 \pm 220$ \\
\hline-1147 & T'T & C-69-1 & 1969 & III C2 Bronze age & 2000 вс* & 1 & $3580 \pm 130$ \\
\hline-1148 & $\mathbf{T} \mathbf{T}$ & C-71-2 & 1971 & III CI Bronze age & $2300 \mathrm{вC} *$ & $2 / 3$ & $3920 \pm 250$ \\
\hline-2302 & $\mathrm{TT}$ & $77-2$ & 1977 & III C High terrace & 2200 вC* & 1 & $3690 \pm 130$ \\
\hline-2301 & TT & $77-1$ & 1977 & III C Hissar & 2200 вс* & 1 & $3620 \pm 130$ \\
\hline
\end{tabular}

* MASCA calibration curve

General Comment (SC): 1st three results of hist period are a little older than expected but may give apparent ages of wooden timbers. Ly-2249 is very different from expected date and may be explained either by re-use of old timber or by sampling problem. As dendrochronol correction of conventional ${ }^{14} \mathrm{C}$ dates was used for determining expected ages, Ly-1147 and -1148 agree with expected range of dates and with pub result, TUNC42: $3625 \pm 71 \mathrm{BP}(\mathrm{R}, 1973, \mathrm{v} 15, \mathrm{p} 596)$. These three results from level of low ancient town while Ly-2302 and -2301 deal with destruction of high terrace, also assumed from III C1/III C2 transition period. Results agree with expected ages and previous results (Deshayes, 1976). Another big timber, charred in same fire dated by Ly-2301 and -2302 gave Gif-3339: $4000 \pm 110$, which also fits if apparent age of biggest timber is considered older.

\section{Iron age}

Ly-1808. En Magne, Chavéria, Jura

$1130 \pm 430$

Human bone from sepulture under Tumulus XIV $\left(46^{\circ} 31^{\prime} \mathrm{N}, 5^{\circ} 33^{\prime}\right.$ E). Coll 1969 and subm 1978 by D Vuaillat, Dir Antiquités Préhist, Besançon. Assoc with Bronze sword from Hallstatt period. (1/5 diluted sample). Comment (DV): for unknown reasons date is completely different from expected age. Stratigraphic data indicates no re-use or rehandling of sepulture occurred, and no contamination of sample may have modified result since it was obtained from only one bone.

\section{Collondon series, Doucier, Jura}

Samples from two places in Les Crevasses site $\left(46^{\circ} 38^{\prime} \mathrm{N}, 5^{\circ} 64^{\prime} \mathrm{E}\right)$. Coll and subm by D Vuailliat.

\section{Ly-2010. Tumulus 1}

Modern

Charcoal from lateral hearth, coll and subm 1979. Assoc with poor industry of Iron age. ( $1 / 2$ diluted sample) 


\section{Ly-2009. Enclos carré}

$1640 \pm 300$

Burned bones and charcoal from cremation area in middle of enclosure. Assoc with Iron age fibulae. (2/5 diluted sample)

General Comment (DV): both dates do not confirm age expected by assoc industries. Ly-2010 rather shows occupation of hearth just before excavation, embedded under colluvia. Ly-2009 indicates carbonaceous remains in enclosure are contaminated by rootlets.

Ly-2300. La Tourette, Pont-du-Château, Puy de Dôme $2060 \pm 120$

Charcoal from dwelling level of Early or beginning of Middle La Tène period $\left(45^{\circ} 37^{\prime} \mathrm{N}, 3^{\circ} 12^{\prime} \mathrm{E}\right)$. Assoc with ceramics and metal artifacts such as Dux fibula, from ca 280 to 250 BC. Coll 1976 by F Malacher and subm 1980 by J P Daugas and F Malacher, Dir Antiquités Préhist Auvergne, Clermont-Ferrand. Comment (JPD): date is a little younger than expected suggesting that charcoal was introduced by colluvia.

Ly-2082. Baccarat, Les Laumes

$2240 \pm 160$

Charcoal from 1.4 to $1.7 \mathrm{~m}$ depth in alluvia of Oze R, Côte d'Or $\left(47^{\circ}\right.$ $32^{\prime} \mathrm{N}, 4^{\circ} 27^{\prime} \mathrm{E}$ ). Coll 1978 by $\mathrm{M}$ Ariente and subm 1978 by J J Puisségur, Inst Geol, Univ Dijon. Assoc with coarse potsherd attributed to Iron age. Comment (JJP): agrees with archaeol attribution.

Ly-1807. Lit de la Saône, Seurres, Côte d'Or

$2510 \pm 130$

Wood from leg of statuette of naked man found by dredging in channel of La Saône R $\left(47^{\circ} 00^{\prime} \mathrm{N}, 5^{\circ} 31^{\prime} \mathrm{E}\right)$. Coll and subm 1977 by L Bonnamour, Mus Denon Châlon-sur-Saône. Comment (LM): conforms with expected age since such votive statues used to be thrown in fountains or rivers from Early Bronze age to end of Roman times, mainly at end of Hallstatt and during La Tène period, when person was represented naked. Late Hallstatt ceramics agrees perfectly with date.

\section{Les Jiraudonnes series, Augères, Creuse}

Charcoal from two tumuli $\left(46^{\circ} 05^{\prime} \mathrm{N}, 1^{\circ} 42^{\prime} \mathrm{E}\right)$. Coll 1975 by $\mathrm{P}$ Léger and subm 1980 by G Mazière, Dir Antiquités Préhist, Limoges.

Ly-2353. Tumulus 1

$2150 \pm 120$

From cremation tumulus containing Late Hallstatt industry.

\section{Ly-2354. Tumulus 2}

$2190 \pm 250$

From tumulus with collective burial containing Late Hallstatt and Early La Tène industries. (11/30 diluted sample)

General Comment (GM): both dates agree with each other but ca $200 \mathrm{yr}$ too young. They do not distinguish between two Iron age periods.

\section{Ly-1862. Tumulus Tugayé I, Ger, Pyrénées \\ Atlantiques}

$$
2470 \pm 300
$$

Burned bones and charcoal from funerary urn in Tugayé $\mathrm{I}\left(43^{\circ} 15^{\prime}\right.$ N, $\left.0^{\circ} 05^{\prime} \mathrm{W}\right)$. Subm 1978 by Coquerel, Tarbes. Comment (RC): despite large dilution of sample (1/5) and large statistical margin, date confirms 
expected age around transition between First and Second Iron ages, which seems to have been delayed in Central Pyrénées massif.

\section{Ly-1971. La Forêt Basse, Saint-Pierre-de-Fursac, Creuse}

$2390 \pm 120$

Charcoal and burned bones from central sepulture "cairn", $2 \mathrm{~m}$ deep, of tumulus $\left(46^{\circ} 09^{\prime} \mathrm{N}, 1^{\circ} 28^{\prime} \mathrm{E}\right)$. Coll and subm 1979 by G Mazière, Dir Antiquités Préhist d'Auvergne, Limousin. Assoc hillock contains potsherds, flints, and Early Iron age sepulture with Iron belt ring. Comment (GM): date indicates re-use of earth sepulture which also contains Neolithic and Bronze age remains.

\section{Ly-2222. Camp de Larina, Annoisin et Chatelans, Isère}

$2420 \pm 110$

Charcoal from hearth in Loc 3 of Camp de Larina site $\left(45^{\circ} 47^{\prime} \mathrm{N}, 5^{\circ}\right.$ $18^{\prime}$ E). Coll and subm 1979 by $\mathrm{H}$ Chatain, Villefontaine, Isère. Comment (HC): date indicates end of Hallstatt period. Hearth, without typical archaeol material must be assoc with several artifacts of Hallstatt period from other areas of site. Ly-2222 is 1st and only date for settlement, which remained occupied from Neolithic to Merovingian times. It may be compared with Ly-880: $2450 \pm 110(\mathrm{R}, 1976, \mathrm{v} 18, \mathrm{p} 72)$, from La Balme site at Sollières-Sardière, Savoie.

\section{Ly-1912. Roja, Castifao, Haute-Corse}

$2420 \pm 180$

Bones from $20 \mathrm{~cm}$ depth in Roja rock shelter $\left(42^{\circ} 30^{\prime} \mathrm{N}, 9^{\circ} 07^{\prime} \mathrm{E}\right)$. Coll 1976 by L Acquaviva, Nice, and subm 1979 by J Jehasse, Dir Antiquités, Corsica. Site is collective sepulture with fairly poor industry probably of First Iron age. Comment (JJ): dates sample at 1000 to $500 \mathrm{BC}$, and seems satisfactory.

\section{Ly-2242. Cami Salié, Pau, Pyrénées Atlantiques $2650 \pm 140$}

Charcoal under funerary urn in Tumulus $1\left(43^{\circ} 19^{\prime} \mathrm{N}, 0^{\circ} 25^{\prime} \mathrm{W}\right)$. Coll 1977 and subm 1978 by G Marsan, Lab Geol Quaternaire, Univ Bordeaux. Assoc with First or Second Iron age industry. Comment (GM): date agrees with possible range of dates for First Iron age (Hallstatt), but precise limit of Second Iron age (La Tène) is not well-defined in region.

\section{Ly-2191. Beauverger, Villeneuve-les-Cerfs, Puy de Dôme}

Charcoal from $1 \mathrm{~m}$ depth in clayey and sandy Tumulus 6 of Beauverger site $\left(46^{\circ} 02^{\prime} \mathrm{N}, 3^{\circ} 20^{\prime} \mathrm{E}\right)$. Coll 1979 and subm 1980 by $\mathrm{D}$ Miallier, Centre Recherches Interdisciplinaires Archéol Anal, Univ Bordeaux. Comment (DM): according to regional context and some assoc potsherds, tumulus may belong to lst Iron age in agreement with result. TL measurements on assoc potsherd are in process; provisional result, calibrated with well-dated samples, is BDX-328: $2384 \pm 180$ BP. Agreement between both dating methods seems perfect. 
Fosse de Caramontron de Sinzelles series, Polignac, Haute Loire

Charcoal from fill of pit outcropping in rd bank $\left(45^{\circ} 04^{\prime} \mathrm{N}, 3^{\circ} 52^{\prime}\right.$ E). Assoc with industry with mixed characteristics of Late Bronze III age and beginning of Iron age, similar to alpine coastal stas. Coll and subm 1978 by J J Houdré and J Vidal, Le Puy.

\section{Ly-2036. 6th and 8th cleaning}

$2410 \pm 130$

From 20 to $30 \mathrm{~cm}$ depth in middle of pit.

\section{Ly-2037. 10th cleaning}

$2520 \pm 120$

From 50 to $55 \mathrm{~cm}$ depth at base of pit.

General Comment (JJH\&JV): both dates attribute Iron age to fill of pit and confirm lasting Late Bronze influences in region. They are much more recent than Late Bronze culture in alpine lakes: see, eg, Ly-17: 2700 $\pm 100 \mathrm{BP}(\mathrm{R}, 1969, \mathrm{v} 11, \mathrm{p} 115)$ from Châtillon coastal sta in Le Bourget lake.

\section{Bronze age}

Ly-2056. Sandgrube, Sierentz, Haut-Rhin

$2550 \pm 100$

Charcoal from 0.8 to $1.1 \mathrm{~m}$ depth in cremation Sepulture 10 of openair site Sandgrube $\left(47^{\circ} 40^{\prime} \mathrm{N}, 7^{\circ} 26^{\prime} \mathrm{E}\right)$. Coll 1978 by J J Wolf and subm 1979 by A Thevenin, Dir Antiquités Préhist, Strasbourg (Wolf, 1978). Comment (AT): date only agrees with expected age of assoc Late Bronze III industry, ca 3000 to $2700 \mathrm{BP}$, if double standard deviation is used.

\section{Ly-2053. Tumulus 22, Kirchlach, Schirrhein,}

Bas-Rhin

Charcoal from $60 \mathrm{~cm}$ depth in hearth $\left(48^{\circ} 48^{\prime} \mathrm{N}, 7^{\circ} 54^{\prime} \mathrm{E}\right)$ assoc with poor ceramic industry attributed to Middle Bronze age. Coll and subm 1978 by A Thevenin. Comment (AT): date is obviously too young; it may indicate reuse of site for Middle age hearth or disturbance of layers by roots.

\section{Kastenwald series, Appenwhir, Bas-Rhin}

Charcoal from cremation sepultures underlying two neighboring hillocks in Kastenwald forest $\left(48^{\circ} 02^{\prime} \mathrm{N}, 7^{\circ} 27^{\prime} \mathrm{E}\right)$. Coll by C Bonnet, S Pouin, and F Lambach and subm 1978 by A Thévenin (Bonnet \& Plouin, 1979).

Ly-2055. Tombe 1

Coll 1975, from Sepulture 1 at $0.1 \mathrm{~m}$ depth, assoc with Middle Bronze age III industry.

\section{Ly-2054. Tombe 5}

Coll 1974 from Sepulture 5 at $1 \mathrm{~m}$ depth, assoc with Middle Bronze age III, a little more recent than Ly-2055.

General Comment (AT): dates confirm both hillocks are contemporaneous but were built during Late Bronze age contrary to archaeol attribution of assoc industry. 
Ly-2325. Chemin de la Pêcherie BCP-55, Berry-auBac, Aisne

Bones from refuse pit of house of Late Bronze age village in Aisne $\mathbf{R}$ valley (see Neolithic series from same valley, below) $\left(49^{\circ} 24^{\prime} \mathrm{N}, 3^{\circ} 53^{\prime} \mathrm{E}\right)$. Coll 1978 and subm 1979 by Unité Recherche Archéol no. 12, Inst Art Archéol, Paris. (11/15 diluted sample). Comment (URA 12): date seems a little too young because of attribution of site to Late Bronze IIb (Hallstatt A2 in German chronology), presumably 3000 to $2100 \mathrm{BP}$.

\section{Ly-1951. Pirogue du Crèt de Chatillon, Sévrier,} Haute-Savoie

Wood from monoxyl barge in oak found at $4 \mathrm{~m}$ depth in Annecy Lake on coastal sta Le Crèt de Chatillon (45 $52^{\prime} \mathrm{N}, 6^{\circ} 08^{\prime} \mathrm{E}$ ). Coll 1979 by $\mathrm{P}$ Persond and subm 1979 by A Bocquet, Centre Préhist Documentation Alpine, Grenoble. Comment (AB): date indicates end of Bronze age in perfect agreement with archaeol material of coastal sta and previous dates from site, ie, Ly-17: $2700 \pm 100(\mathrm{R}, 1969, \mathrm{v}$ 11, p 115) and Ly-274: $2670 \pm$ $110(\mathrm{R}, 1971, \mathrm{v} 13$, p 57).

Ly-1986. Salle des Gardes, Caen, Calvados

$\mathbf{3 0 3 0} \pm \mathbf{4 5 0}$

Human bones from excavation under so-called Salle des Gardes bldg in medieval castle $\left(49^{\circ} 10^{\prime} \mathrm{N}, 0^{\circ} 22^{\prime} \mathrm{W}\right)$. Coll 1976 by C Pilet, Dir Antiquités Hist Caen, and subm 1979 by G Verron, Dir Antiquités Préhist, Caen. (1/2 diluted sample). Comment (GV): dates to Bronze age skeleton which was lying without assoc industry under level from beginning of Roman period (1st century BC).

Ly-1866. Grotto Linars, Rocamadour, Lot

$3080 \pm 240$

Human bones from Linars sepulchral grotto $\left(44^{\circ} 48^{\prime} \mathrm{N}, 1^{\circ} 37^{\prime} \mathrm{E}\right)$. Coll by L Genot, Leyme, Lot, and subm by A Cogoluenhes, Dept Geol, Univ Lyon. Assoc with ceramic industry of Les Champs d'Urnes culture of Late Bronze age. Comment (AC): date agrees with archaeol estimate and confirms that site was not disturbed as was previously thought.

\section{Ly-2244. Le Verger, Saint-Romain, Côte d'Or}

$3540 \pm 230$

Charcoal from base of Early Bronze age layer $\left(46^{\circ} 59^{\prime} \mathrm{N}, 4^{\circ} 43^{\prime} \mathrm{E}\right)$ (see also Neolithic layers of same site, below). Coll and subm 1979 by S Grappin, Dir Antiquités, Dijon. (2/5 diluted sample). Comment (SG): confirms archaeol attribution to Early Bronze age.

\section{Ly-1773. Camp de Chassey, IL 56 TP, Chassey,} Saône et Loire

Charcoal from piling hole of rampart of fortified plateau $\left(45^{\circ} 53^{\prime} \mathrm{N}\right.$, $4^{\circ} 46^{\prime} \mathrm{E}$ ). Rampart is not assoc with industry but is assumed to be from protohistoric period (Bronze or Hallstatt). Coll 1972 and subm 1978 by J P Thévenot, Dir Antiquités Préhist, Dijon. Comment (JPT): in expected range, dates rampart most probably to Middle Bronze age as confirmed by stratigraphy. 


\section{Ly-1831. La grotte de Pégourié 1725, Caniac du Causse, Lot}

Charcoal from hearth found in one of upper layers of grotto fill $\left(44^{\circ}\right.$ $37^{\prime} \mathrm{N}, 1^{\circ} 39^{\prime} \mathrm{E}$ ). Coll 1977 and subm 1978 by R Séronie-Vivien, Le Bouscat, Gironde. Presumably Chassean potsherds were found close to hearth, but site may have also been used during Middle or Early Bronze ages. (2/5 diluted sample). Many other dates were obtained from lowest layers of site (below). Comment (RS-V): as expected, dates boundary between Early and Middle Bronze ages. Cf unpub results from Les Claups grotto, Gif-3568: $3210 \pm 110$ and pub results from Layers 2 and 3 of Le Noyer grotto, Gif-1631: $3150 \pm 110$, and Gif-1159: $3250 \pm 110$ (R, 1972, v 14, p 288).

\section{Stathmos Aggistas series, Serres, E Macedonia, Greece}

Charcoal from two locs in $\mathrm{N}$ excavation of Stathmos Aggistas site $\left(41^{\circ} 00^{\prime} \mathrm{N}, 23^{\circ} 57^{\prime} \mathrm{E}\right)$. Coll 1977 by Ch Koukouli-Chrysanthaki, Kavala Archaeol Mus, and subm 1978 by J Deshayes, Paris. Site is tell underlying tumulus of Macedonian grave. Tell presumably belongs to Macedonian culture of final Late Bronze age, 1400-1100 BC (Koukouli-Chrysanthaki, 1980). (1/2 and $3 / 5$, respectively diluted samples)

$$
\begin{array}{lll}
\text { Ly-1778. } & 2.8 \mathrm{~m}, \mathrm{~N} \text { sec } & 3700 \pm 270 \\
\text { Ly-1779. } & \text { Pit } 3, \mathrm{~N} \text { sec } & 4300 \pm 230
\end{array}
$$

General Comment (CKC): since MASCA calibration of both dates set them at end of 3rd millennium $\mathrm{BC}^{*}$, they are much older than expected. Oldest layer of site, that of Ly-1748, is archaeol dated by Mycenean vases to 14th century BC. Ly-1748 comes from more recent layer. Organic material found in Pit 3 (Ly-1749) was previously dated by Zentral Inst Berlin to $940 \pm 65 \mathrm{BC}$ (uncalibrated and unpub).

\section{Ly-1806. Tumulus F 16, Lamarque-Pontacq, Hautes- Pyrénées}

$3730 \pm 190$

Charcoal from cremation area of F 16 tumulus $\left(43^{\circ} 12^{\prime} \mathrm{N}, 0^{\circ} 07^{\prime} \mathrm{W}\right)$. Coll 1966 and subm 1977 by R Coquerel, Tarbes. Although without assoc industry site may be compared with La Gourgue d'Asque site, HautesPyrénées (Clot, Coquerel, \& Omnès, 1978) previously dated by Ly-1053: $3800 \pm 40$ (R, 1978, v 20, p 40). Comment (RC): date confirms contemporaneity with Ly-1053; both dates indicate relatively old age of cremations in Central Pyrénées (Coquerel, 1966).

\section{Ly-2180. Berges de l'Artière, Les Martres d'Artière, Puy de Dôme}

$4200 \pm 160$

Animal bones from left bank of Artière R ( $\left.45^{\circ} 50^{\prime} \mathrm{N}, 3^{\circ} 04^{\prime} \mathrm{E}\right)$. Coll 1978 and subm 1980 by J P Daugas, Dir Antiquités Préhist d'Auvergne, Clermont-Ferrand, and J P Raynal, Inst Quaternaire Bordeaux, Talence. (4/5 diluted sample). Outcropping sediments were formed by filling of ancient river channel. Level also contains "en barbelé" decorated ceramic, well-known in S France and attributed to beginning of Early Bronze age. 
Comment (JPD\&JPR): date is a little older than expected by assoc industry and shows ancient river channel was filled from Late Neolithic to Early Bronze ages.

\section{Ly-1868. Grotte de l'Homme-Mort, Lomné, Hautes- \\ Pyrénées}

$3760 \pm 150$

Human bones from debris of ancient excavation in sepulchral gallery of Lomné grotto near Lannemezan $\left(43^{\circ} 0^{\prime} \mathrm{N}, 0^{\circ} 17^{\prime} \mathrm{E}\right)$. Coll and subm 1979 by J Omnès, Lourdes. Comment (JO): date agrees with presumed Bronze age and assoc industry, stabber and nail-decorated ceramics (Omnès, 1981). Cf Ly-1904 from Artigaou grotto (below).

\section{E. Chalcolithic/Neolithic}

\section{Dolmen de Mourioux series, Mourioux, Creuse}

Charcoal from soil of dolmen in Bois de Mourioux $\left(46^{\circ} 04^{\prime} \mathrm{N}, 1^{\circ} 39^{\prime}\right.$ E). Coll by R Credot and M Dominique and subm 1978 by G Mazière, Dir Prehist, Limoges. Assoc with Neolithic industry.

Ly-1968. No. 1

$240 \pm 160$

Coll 1976. (9/10 diluted sample)

Ly-1969. No. 2

$2010 \pm 130$

Coll 1977.

General Comment (GM): dates indicate re-use of sample as frequently happens in megalithic monuments, which therefore cannot be dated in this manner.

\section{Santourin series, Billième, Savoie}

Charcoal from two levels $\left(45^{\circ} 49^{\prime} \mathrm{N}, 5^{\circ} 23^{\prime} \mathrm{E}\right)$ coll 1978 and subm 1979 by L Lagier-Bruno, Yenne, Savoie. Site is mainly stone circles which may have been sheepfolds or Neolithic dwellings (Lagier-Bruno, 1981). (Ca $1 / 5$ diluted sample)

\section{Ly-2287. Level II}

$3550 \pm 220$

Ly-2288. Level III

$4340 \pm 290$

General Comment (LLB): previous date on Level I, Ly-1604: $2240 \pm 260$ $(\mathrm{R}, 1979, \mathrm{v} 22, \mathrm{p} 432)$ indicates dates are in stratigraphic order; also indicates Late-Neolithic/Early-Bronze transition in agreement with presumed ages of other megalithic monuments.

\section{La Touvière series, Thoyes, Arbignieu, Ain}

Samples from so-called $\mathrm{La}$ Couche Brune level at two locs in rock shelter $\left(45^{\circ} 45^{\prime} \mathrm{N}, 5^{\circ} 39^{\prime} \mathrm{E}\right)$. Coll and subm 1978 and 1979 (Ly-2259) by $\mathrm{R}$ Vilain, Dept Geol, Univ Lyon. Assoc with uncharacteristic industry which may be Chalcolithic.

\section{Ly-1974. F-4}

$1010 \pm 130$

Charcoal from Sq F-4 in front part of rock shelter. 
Charcoal from Sqs C-4 and C-5 in front part of rock shelter. (2/3 diluted sample)

Ly-2259. Sepulture

Human bones from multiple sepulture in bottom of rock shelter. ( $7 / 10$ diluted sample)

General Comment (RV): dates from charcoal are too young for assoc industry in level. They indicate extensive influence of modern hearth found near excavated sqs. Date on bones is plausible for assoc industry, which may either belong to Bronze age or, more probably, is older but redeposited by burials (Morelon, 1974).

Ly-2214. Frépestel, Meyrueis, Lozère

$1660 \pm 160$

Human bones from under flagstone-covered sepulture $\left(44^{\circ} 12^{\prime} \mathrm{N}, 3^{\circ}\right.$ $\left.27^{\prime} \mathrm{E}\right)$, assoc with industry most probably from Chalcolithic. Coll 1979 by G Fagès and subm 1980 by A Cogoluènhes. (11/15 diluted sample). Comment (GF): much younger than expected; dates show disturbance in sepulture suspected from bone distribution.

\section{Ly-2245. Hermanky, Ceska Lipa, Bohemia,} Czechoslovakia

Charcoal from Hermanky rock shelter $\left(50^{\circ} 43^{\prime} \mathrm{N}, 14^{\circ} 35^{\prime} \mathrm{E}\right)$. Coll and subm 1979 by J Svoboda, Univ Brno. Samples were found in Neolithic living areas in rock shelter (Svoboda, 1979). (7/30 diluted sample). Comment (JS): date is much too young; may be contaminated by recent roots.

\section{Ly-2295. Aven de Jacques, Lussac, Ardèche}

$3660 \pm 130$

Human bones from Jacques sepulchral grotto-aven $\left(44^{\circ} 37^{\prime} \mathrm{N}, 4^{\circ} 29^{\prime}\right.$ E). Coll 1979 and subm by A Cogoluènhes, Dept Geol, Univ Lyon. Assoc with Fontbouise Chalcolithic industry. Comment (AC): date is younger than generally thought for Fontbouise culture, but it may fit in usual range with double statistical margin.

\section{Ly-1904. Grotte de Las Crouts d'Artigaou, Esparros, Hautes Pyrénées}

Human bones from sepulchral recess near Lannemezan $\left(43^{\circ} 02^{\prime} \mathrm{N}\right.$, $0^{\circ} 05^{\prime} \mathrm{E}$ ). Coll and subm 1978 by J Omnès, Lourdes. Comment (JO): date agrees with Chalcolithic age presumed by poor assoc ceramic industry. $C f$ Ly-1868 (above) from Lomné sepulchral grotto (Omnès, 1981).

\section{Ly-1750. Bré-Sourbette, Veyreau, Aveyron}

$3800 \pm 130$

Bones from sepulchral grotto $\left(44^{\circ} 06^{\prime} \mathrm{N}, 3^{\circ} 02^{\prime} \mathrm{E}\right)$ assoc with Late Rodezian Chalcolithic or Chalcolithic with "Céramiques à triangles hachurés" industry and with fluted point lodged in bone (Fagès \& MourerChauviré, in press). Coll 1978 by G Fagès, Florac, and subm 1978 by C Mourer-Chauviré, Dept Geol, Univ Lyon I. Comment (GF\&CMC): date is younger than expected from Late Neolithic assoc industry previously dated in La Treuille grotto: 3 Gif dates са 4600 вр (R, 1974, v 16, p 25), 
but agrees with dates of other sites of same industry: Sargel grotto, Gif328: $3710 \pm 180$ (R, 1970, v 12, p 423), Les Côtes of Roquefort, Gif-37: $3930 \pm 150(\mathrm{R}, 1966, \mathrm{v} 8, \mathrm{p} 130)$, and La Fajole, Ly-2213 (below).

\section{Ly-2213. La Fajole, Vebron, Lozère}

Human bone from megalithic sepulture Galdri $\left(44^{\circ} 17^{\prime} \mathrm{N}, 3^{\circ} 32^{\prime} \mathrm{E}\right)$ assoc with so-called Rodezian Chalcolithic/Early Bronze industry. Coll and subm by $\mathrm{G}$ Fagès and subm 1980 by $\mathrm{A}$ Cogoluènhes. Comment (GF): agrees with expected age and another date from same culture in region, Ly-1750 from Bré Sourbette site (above).

\section{Ly-2305. Pirogue de Meimart, Brisson-Saint-Innocent, Savoie}

Wood from monoxyl barge in oak found $2 \mathrm{~m}$ deep on bottom of Le Bourget Lake $\left(45^{\circ} 44^{\prime} \mathrm{N}, 5^{\circ} 52^{\prime} \mathrm{E}\right)$. Coll 1980 by R Castel and subm 1980 by A Bocquet, Centre Préhist Documentation alpine, Grenoble. Comment (AB): dates barge to Late Neolithic and three centuries later than fragment of wooden cup of Saône et Rhône culture from same site, Ly-190: $4060 \pm 120(\mathrm{R}, 1971, \mathrm{v} 13$, p 57).

\section{Ly-1989. Chapeau-Rouge, Menetrol, Puy-de-Dôme $\quad 3750 \pm \mathbf{2 4 0}$}

Human bones from probable Neolithic sepulture $\left(45^{\circ} 52^{\prime} \mathrm{N}, 3^{\circ} 18^{\prime}\right.$ E). Coll and subm 1979 by J P Daugas and J P Raynal. (2/3 diluted sample). Comment (JPD\&JPR): date may be younger than expected because of chemical composition of embedding sediment, Terre Noire de Limagne, which is black earth rich in organic matter (Daniel et al, 1979).

\section{Chalain lake series, Doucier, Jura}

Wood from two foundation pilings of two eroded coastal stas of W side of Chalain Lake $\left(46^{\circ} 40^{\prime} \mathrm{N}, 5^{\circ} 48^{\prime} \mathrm{E}\right)$. Coll and subm 1978 by $\mathrm{D}$ Vuaillat, Dir Antiquités, Préhist Centre, Besançon.

\section{Ly-2007. Sta 5}

Ly-2008. Sta 6
$4250 \pm 130$

$4170 \pm 140$

General Comment (DV): no artifacts remained in sta although three other sites were discovered and dated in E part of lake; 1st one from Middle Neolithic culture near Les Roseaux inlet, at Fontenu, Ny-143: $5790 \pm$ 220 and $\mathrm{Ny}-144: 5850 \pm 180$ (R, 1974, v 16, p 120); others, stas 1 and 2 from Late Neolithic Saône et Loire culture, Gif-2637: 4220 \pm 140 and Gif-2638: $4280 \pm 180$ (unpub). Dates confirm contemporaneity of stas 5 and 6 with stas 1 and 2 and exclude their attribution to Bronze age.

\section{Ly-2078. Croix-Tombée cemetery, Pérouges, Ain $4060 \pm 100$}

Human bones from grave of Gallo-Roman cemetery $\left(45^{\circ} 53^{\prime} \mathrm{N}, 5^{\circ} 11^{\prime}\right.$ E). Coll 1979 by J L Challard and subm 1979 by A Cogoluenhes, Dept Geol, Univ Lyon. This grave was laid at lower level than other graves with different orientation. Comment $(\mathrm{AC})$ : date confirms expected old age of this isolated grave which may belong to Neolithic or Chalcolithic periods because of assoc flints. 
Charcoal from fill of small calcareous cavity on hill $\left(48^{\circ} 04^{\prime} \mathrm{N}, 2^{\circ} 53^{\prime}\right.$ E). Coll 1977 and subm 1980 by A Aubourg, Amilly, Loiret. Assoc with uncharacteristic industry, maybe from Neolithic. (4/5 diluted sample). Comment (AA): dates cavity fill to Late Neolithic.

\section{Ly-1903. Grotte du Castillet, Lourdes, Hautes-}

\section{Pyrénées}

$4380 \pm 140$

Human bone from sepulchral gallery $\left(43^{\circ} 06^{\prime} \mathrm{N}, 0^{\circ} 07^{\prime} \mathrm{E}\right)$. Coll and subm 1978 by J Omnès. Comment (JO): date agrees with Late Neolithic attribution with poor industry mainly of bone arrows and fingers or naildecorated ceramics.

\section{Ly-1962. Grotte de la Gardette, Labastide de Virac, Gard}

Human bones from Late Neolithic or Chalcolithic sepulchral grotto, Ardèche $\left(44^{\circ} 21^{\prime} \mathrm{N}, 4^{\circ} 24^{\prime} \mathrm{E}\right)$. Coll 1977 by $\mathrm{P}$ Perreve and subm 1978 by A Cogoluènhes. Comment (AC): agrees with expected age and comparable to other dates of Late Neolithic Ferrières in region, eg, in Traves grotto at Montclus, Gard, Gif-1909: $4260 \pm 140$ (R, 1974, v 16, p 31).

Ly-1963. Abauntz, Level b, Arraiz, Navarra, Spain $4240 \pm 140$

Charcoal from Level b in Abauntz grotto $\left(43^{\circ} 01^{\prime} \mathrm{N}, 1^{\circ} 42^{\prime} \mathrm{W}\right)$. Coll 1976 and subm 1978 by P Utrilla-Miranda, Univ Zaragoza. Assoc with burned human bones and with Late Neolithic or Chalcolithic industry. Comment (PUM): date agrees with expected value; $c f$ date from Level IIIB in Los Husos grotto at Elvillar, Alava, Spain, I-3985: $3920 \pm 100$ (unpub). Two other dates by Teledyne Isotope lab are from Neolithic Level b4, I-11,309: $5390 \pm 120$ and Level c, I-11,537: $6910 \pm 450$ (UtrillaMiranda, 1980).

Ly-1941. Le Jas des Chèvres, Allan, Drôme

$4390 \pm 160$

Charcoal from $40 \mathrm{~cm}$ depth from open-air site $\left(44^{\circ} 28^{\prime} \mathrm{N}, 4^{\circ} 48^{\prime} \mathrm{E}\right)$. Coll 1978 and subm 1979 by A Beeching, Dir Antiquités Préhist, Lyon. Assoc with a Pre-Campaniforme Chalcolithic industry (Beeching, 1980). (9/10 diluted sample). Comment (AB): agrees with expected age, indicating relatively old age for Chalcolithic industry, but compared with unpub result on charcoal from Bruyères site at Saint Julien de Peyroles, Gard, MC-976: $4225 \pm 80$ (Gilles, 1975).

\section{Ly-2348. Beaulieu, Bardouville, Seine Maritime $\quad 4550 \pm 130$}

Human bones from collective sepulture of Beaulieu quarry $\left(49^{\circ} 26^{\prime}\right.$ N, $0^{\circ} 51^{\prime}$ E). Coll 1966 by R Caillaud and E Lagnel and subm 1980 by $\mathrm{G}$ Verron and J Dastugue, Caen. Assoc with Late Neolithic industry, SeineOise-Marne (SOM) (Caillaud and Lagnel, 1967). Comment (GV): dates generally obtained for SOM culture are younger but those from Videlles site, GrN-4676: $4500 \pm 50$ and GrN-4675: $4500 \pm 60$ (R, 1967, v 9, p 133) are close to present one. 


\section{Ly-1738. La Pierre Godon, Tillay le Peneux, Eure et Loir}

$4550 \pm 150$

Bones from $90 \mathrm{~cm}$ depth under pavement of access passage of dolmen under tumulus at Soignolles $\left(48^{\circ} 10^{\prime} \mathrm{N}, 1^{\circ} 47^{\prime} \mathrm{E}\right)$. Coll and subm 1978 by G Richard, Orléans. Comment (GR): date indicates 1st occupation of tumulus occurred during Late/Middle (Chassean) Neolithic period. $C f$ date from Fort Harrouard, nearby, Gsy-97: $4400 \pm 135$ (R, 1966, v 8, p 131); 2nd occupation of site occurred at end of Neolithic, from ca 4300 to $3800 \mathrm{BP}$, SOM culture with Campaniforme influences (Richard, 1980).

\section{Le Fournet series, Montmaur, Drôme}

Table 20 lists samples of human bones from several locs in sepulchral grotto $\left(44^{\circ} 41^{\prime} \mathrm{N}, 5^{\circ} 20^{\prime} \mathrm{E}\right)$. Coll 1966 by $\mathrm{A}$ Heritier and subm by $\mathrm{A}$ Cogoluènhes.

TABLE 20

Le Fournet

\begin{tabular}{rccc}
\hline Sample no. & Bone ref & Dilution ratio & Age BP \\
\hline Ly-2433 & $11 / 1$ & $1 / 2$ & $3590 \pm 180$ \\
-2431 & $10 / 17$ & $1 / 3$ & $3840 \pm 190$ \\
-2432 & $6 / 1$ & $9 / 10$ & $4240 \pm 160$ \\
-2434 & $10 / 1$ & 1 & $5440 \pm 130$ \\
\hline
\end{tabular}

General Comment (AC): from previous series of 4 dates from same site ( $R, 1979, v 21, \mathrm{p} 436)$ ages at end of Neolithic were expected. These 4 new results show grotto was used from Middle Neolithic (Ly-2434) to Bronze age (Ly-2433). Assoc industry for whole bones is Chassean (agreeing, eg, with Ly-2434) or Chalcolithic (Ly-2431, -1178, -1733). Both series indicate need for many results to determine total duration of occupation (Cogoluènhes, 1977).

\section{Ly-1688. Le Brudoux, Plan de Baix, Drôme}

$4710 \pm 150$

Human bones from sepulchral grotto $\left(44^{\circ} 49^{\prime} \mathrm{N}, 5^{\circ} 10^{\prime} \mathrm{E}\right)$. Coll by M C Haze and subm 1978 by A Cogoluènhes. Assoc with heterogeneous, peor industry of Chalcolithic. Comment (AC): date is too old for Chalcolithic but site was disturbed.

\section{Ly-2518. La Montagne de Comin, Bourg-et-Comin, Aisne}

Charcoal from fire layer underlying Middle Neolithic (Chassean or Michelsberg) occupation level on spur at top of plateau $\left(49^{\circ} 25^{\prime} \mathrm{N}, 3^{\circ} 40^{\prime}\right.$ E). Coll and subm 1981 by CNRS Unit 12. Comment (URA 12): dated to solve complex stratigraphic problem of clayey levels; date confirms fire layer is contemporaneous with Neolithic occupation since result is close to other results for Michelsberg industries in Aisne R Valley (Ly-2328 and -2334, below). 


\section{La Roberte series, Chateauneuf du Rhône, Drôme}

Bones from two trenches of Chassean site $\left(44^{\circ} 32^{\prime} \mathrm{N}, 4^{\circ} 43^{\prime} \mathrm{E}\right)$. Subm 1979 by A Beeching. This was open-air site presently covered by $10 \mathrm{~m}$ of earth (Beeching \& Thomas-Beeching, 1975).

Ly-2076. St 4, 72P

$4830 \pm 150$

From 60 to $65 \mathrm{~cm}$ depth in Pit 4 . Coll 1977 by J Thomas-Beeching, Lyon. (4/5 diluted sample)

Ly-2075. St 2, $1 P$

$4970 \pm 200$ sample)

From fill of Pit 2. Coll 1976 by M Lambert, Montélimar. (3/5 diluted

General Comment (AB): both dates are in mutual statistical margins but some differences in Chassean typology suggest that Pit 2 (Ly-2075) should be a little older than Pit 4 (Ly-2076). Both dates are closer to generally obtained value for Middle Neolithic in region than those obtained in neighboring Chassean site, Francin, Savoie, Lv-389: $3870 \pm 170$ and Lv-390: $4300 \pm 75$ (R, 1970, v 12, p 554).

\section{Chassey camp series, Chassey, Saône et Loire}

Table 21 lists samples of charcoal from Neolithic Chassean layers $\left(46^{\circ} 53^{\prime} \mathrm{N}, 4^{\circ} 46^{\prime} \mathrm{E}\right)$. Coll 1977 and subm 1978 by J P Thévenot.

TABLE 21

Chassey Camp

\begin{tabular}{cllcc}
\hline Sample no. & \multicolumn{2}{c}{ Sample } & Sq and level & Age BP \\
\hline Ly-1767 & Chassey 1 XLII-56 & F 8 & $5220 \pm 140$ \\
-1771 & Chassey 2 XLIII-54 & F 8 & $5380 \pm 160$ \\
-1768 & Chassey 3 XLII-56 & TC 9 & $5660 \pm 150$ \\
-1769 & Chassey 4 XLIII-56-57 & A 9 & $5540 \pm 120$ \\
-1770 & Chassey 5 XLII-55 & 4849 & $5380 \pm 140$ \\
-1772 & Chassey 6 L-56 & Diaclase & $5700 \pm 150$ \\
\hline
\end{tabular}

General Comment (JPT): dates agree with stratigraphy and date Level 8 at ca $5300 \mathrm{BP}$ and Level 9 at ca $5600 \mathrm{BP}$. Ly- 1770 is ca $300 \mathrm{yr}$ too young but sample contained large amount of roots. Ly-1772 comes from small zone containing intrusive sediments between two diaclases and industry may be either from Level 8 or 9 and re-used (Thévenot, 1978). Ly-1772 shows date of zone close to Level 9 and does not confirm re-use.

\section{Ly-1791. Collective sepulture, Abri Moula, Soyons,} Ardèche

$5660 \pm 140$

Human bones from remains of multiple sepulture embedded in sediments deposited on upper part of fill of Moula shelter $\left(44^{\circ} 53^{\prime} \mathrm{N}, 4^{\circ} 50^{\prime}\right.$ E). Coll 1972 by Crouzet Archaeol Club and subm 1978 by P Payen, Valence, and A Cogoluènhes. Assoc with poor industry of ceramics and flint of Middle Neolithic. Comment (PP): Chassean date agrees with assoc industry. 


\section{Les Rivaux Loc 1 sommet, Espaly-Saint-Marcel, Haute-Loire}

Table 22 lists samples of charcoal (all but Ly-2194, which is animal bones) from several stratigraphic units (hearths or dwelling levels) of Neolithic-Chassean open-air site $\left(45^{\circ} 03^{\prime} \mathrm{N}, 3^{\circ} 51^{\prime} \mathrm{E}\right)$. Coll and subm by J P Daugas, Dir Antiquités Préhist Auvergne, Clermont-Ferrand.

TABLE 22

Les Rivaux

\begin{tabular}{rcclll}
\hline $\begin{array}{c}\text { Sample } \\
\text { no. }\end{array}$ & $\begin{array}{c}\text { Strat } \\
\text { unit }\end{array}$ & $\begin{array}{c}\text { Colln } \\
\text { date }\end{array}$ & $\begin{array}{c}\text { Dilution } \\
\text { ratio }\end{array}$ & $\begin{array}{c}\text { Assoc } \\
\text { industry }\end{array}$ & Age BP \\
\hline Ly-2303 & $2 \mathrm{e} / 2 \mathrm{a}$ & 1971 & $2 / 5$ & Late Chassean & $4240 \pm 200$ \\
-1349 & CCP 2 & 1974 & $1 / 3$ & Late Chassean & $4540 \pm 210$ \\
-2083 & $\mathbf{3}$ & 1970 & $1 / 2$ & Chassean & $4670 \pm 190$ \\
-2304 & $4 \mathrm{~b}$ & 1973 & $5 / 6$ & Chassean & $4530 \pm 140$ \\
-2289 & $4 \mathrm{a}$ & 1973 & $1 / 6$ & Chassean & $4790 \pm 260$ \\
-2246 & 5 & 1973 & $2 / 3$ & Chassean & $4740 \pm 140$ \\
-2247 & CAC & 1973 & $7 / 30$ & Chassean & $5020 \pm 200$ \\
-2084 & $\mathbf{6}$ & 1973 & $2 / 5$ & Chassean & $5340 \pm 190$ \\
-1348 & $6.4 / 6.5 / F .7$ & 1974 & $1 / 3$ & Chassean & $5600 \pm 210$ \\
-2194 & CM4 & 1973 & 1 (bone) & Chassean & $5030 \pm 100$ \\
-1596 & BdF la & 1974 & $1 / 2$ & Early Chassean & $5200 \pm 250$ \\
-1987 & BdF 2 & 1974 & $3 / 20$ & Early Chassean & $5310 \pm 470$ \\
\hline
\end{tabular}

General Comment (JPD): despite small amount of material, series agrees with expected values. Deepest samples, Ly-1596 and -1987, assoc with Early Chassean were expected to be older but contamination by recent roots developed at this level, BdF, and Ly-1987 is possible, ca 6000 BP, taking into account $2 \sigma$ statistical margin. Ly-2194 is also too young but it comes from pit which may have been mixed with heterogeneous material. All dates confirm long duration of site occupation (ca $1000 \mathrm{yr}$ ) and contemporaneity with eponymic Chassey site (above) (Daugas et al, 1980).

\section{La Baume series, Arlempdes, Haute Loire}

Charcoal from 1.5 to $1.8 \mathrm{~m}$ depth in layers with Mesolithic industry

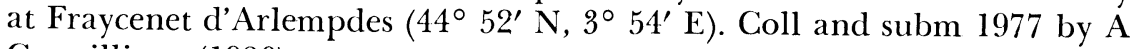
Cremillieux (1980).

\section{Ly-1864. 11}

$5290 \pm 300$

Sample from topmost level. (2/5 diluted sample)

\section{Ly-1865. 13}

$4560 \pm 930$

Sample from deepest level. (1/15 very diluted sample)

General Comment (AC): dates do not fit assoc industry and show that charcoal came from upper level with Neolithic industry. Average date, Ly-1864/1865: $5180 \pm 280$ agrees with two dates in same region, La Roche Dumas, Arsac en Velay, Ly-1588: $5120 \pm 320$ and Le Chambon, Goudet, Ly-1549: $5160 \pm 250(\mathrm{R}, 1979, \mathrm{v} 21, \mathrm{p} 437)$ of Chassean industry. Cf also Les Riveaux series (above).

\section{La Vergentière series, Cohons, Haute-Marne}

Samples from excavation pit S1 in fortified promontory of La Vergentière $\left(47^{\circ} 46^{\prime} \mathrm{N}, 5^{\circ} 20^{\prime} \mathrm{E}\right)$. Assoc with Bourguignon Middle Neolithic 
industry with Cortaillod and Michelsberg characteristics. Coll 1977 and subm 1978 by L Lepage, Saint-Dizier.

\section{Ly.1859. S1-A8}

$5230 \pm 300$

Bones from level underlying archaeol layer. (1/3 diluted sample)

\section{Ly.1860. S1-A17}

$5350 \pm 270$

Charcoal from level underlying limed central part of rampart of buttress. ( $1 / 2$ diluted sample)

General Comment (LL): both dates are in statistical range of each other, weighted average, Ly-1859/1860: $5300 \pm 210$. Building of rampart was contemporaneous with Middle Neolithic occupation (Lepage, 1980).

\section{Grotte de la Pyramide series, Penne, Tarn}

Charcoal from two levels in Loc 3, which opens into archaeol fill of grotto at Le Travers de Janoye $\left(44^{\circ} 05^{\prime} \mathrm{N}, 1^{\circ} 43^{\prime} \mathrm{E}\right)$. Coll and subm by J Lautier, Albi. Both levels contain Chassean industry (Lautier, 1982).

Ly-1867. Layer 6E

$\mathbf{5 4 9 0} \pm \mathbf{3 1 0}$

From Layer 6E, Sq K8. Coll 1973, subm 1977. (3/10 diluted sample)

Ly-1408. Layer 6A

$4750 \pm 270$

From Layer 6A, Sq J11. Coll 1976, subm 1977. (1/3 diluted sample)

General Comment (JL): dates fit Chassean industry which lasted for at least $800 \mathrm{yr}$, but for unknown reason, sample from lowest layer gives younger result. Cf dates from two sites with same Chassean industry, Le Noyer grotto at Esclauzels, Lot, Gif-1633: $500 \pm 130$ (R, 1972, v 14, p 289) and Sargel grotto at Saint-Rome-de-Cernon, Aveyron, Gif-445: $4570 \pm 150$ (R, 1966, v 8, p 130).

\section{Le Verger series, Saint-Romain, Côte d'Or}

Charcoal from two neighboring sites in hearth area in Neolithic part of Le Verger site $\left(46^{\circ} 59^{\prime} \mathrm{N}, 4^{\circ} 43^{\prime} \mathrm{E}\right.$ ), (see Ly-2244, above, from Bronze age layer of site). Coll and subm 1978 (Ly-1985) and 1979 (Ly-2243) by S Grappin, Dijon. Assoc with not well-defined Middle Neolithic industry.

$\begin{array}{ll}\text { Ly-1985. } & 1-2 \\ \text { Ly-2243. } & 3\end{array}$

$\mathbf{5 5 9 0} \pm 130$

$5860 \pm 170$

General Comment (SG): Ly-1985 is contemporaneous with Middle Neolithic Chassey camp (above). Ly-2243 seems too old for Middle Neolithic industry in region.

\section{Ly-2195. Grotte La Balme d'Epy, Jura}

$5640 \pm 200$

Human bones from upper part of fill of grotto $\left(46^{\circ} 23^{\prime} \mathrm{N}, 5^{\circ} 25^{\prime} \mathrm{E}\right)$. Coll 1975 and subm 1979 by A Cogoluènhes. Assoc with Chalcolithic industry. (1/5 diluted sample). Comment (AC): date is a little older than expected, but compatible with less characteristic industry. Previous date from same grotto dated to end of Würmian, Ly-362: $20,300+1900$ (R, 1973, v 15, p 516). 
Ly-2077. Grande Barme de Savigny, La Biolle, Savoie $5010 \pm 140$

Bones from Level $1 \mathrm{Ib}, 70 \mathrm{~cm}$ deep in grotto $\left(45^{\circ} 44^{\prime} \mathrm{N}, 5^{\circ} 55^{\prime} \mathrm{E}\right)$. Coll 1977 by J Thomas-Beeching and subm 1979 by A Beeching. Assoc with atypical industry of Early Neolithic/Middle Neolithic transition, presumed age of which is uncertain but may be same as Cortaillod culture (Beeching, 1979). Comment (AB): date is a little younger than expected but indicates range generally given for Middle Neolithic period in region.

Ly-1766. Port Renard, Vinneuf, Yonne

$3130 \pm 150$

Charcoal from Hearth 29, Loc XIII (48 $\left.38^{\circ} 1^{\prime} \mathrm{N}, 3^{\circ} 28^{\prime} \mathrm{E}\right)$. Coll 1976 by A Carré and subm by J $\mathrm{P}$ Thévenot. Sample is from entrance of hearth in hut of Cerny culture (Middle Neolithic). Comment (JPT): date is much too young and may be explained by roots that penetrated archaeol level as it was close to surface.

\section{Vallée de l'Aisne series, Aisne}

Table 23 lists samples of bones from refuse pits of Early and Middle Neolithic villages in Aisne R valley. Coll from 1975 to 1981 and subm by Unité Recherche Archéol no. 12, Paris, at following sites: la Croix-Maigret (BCM) near Berry-au-Bac $\left(49^{\circ} 24^{\prime} \mathrm{N}, 3^{\circ} 53^{\prime} \mathrm{E}\right)$, Les Jombras (CLJ) near Concevreux $\left(49^{\circ} 23^{\prime} \mathrm{N}, 3^{\circ} 48^{\prime} \mathrm{E}\right)$, Les Fontenettes (CCF), near Cuiry-lesChaudardes $\left(49^{\circ} 23^{\prime} \mathrm{N}, 3^{\circ} 45^{\prime} \mathrm{E}\right)$, Derrière le Village (MDV) near Menneville $\left(49^{\circ} 25^{\prime} \mathrm{N}, 4^{\circ} 01^{\prime} \mathrm{E}\right)$, les Grandes Grèves (VSG), near Villeneuve-StGermain $\left(49^{\circ} 24^{\prime} \mathrm{N}, 3^{\circ} 22^{\prime} \mathrm{E}\right)$.

TABLE 23

Aisne River Valley

\begin{tabular}{|c|c|c|c|c|c|}
\hline $\begin{array}{c}\text { Sample } \\
\text { no. }\end{array}$ & Ref & $\begin{array}{c}\text { Colln } \\
\text { date }\end{array}$ & $\begin{array}{l}\text { Dilution } \\
\text { ratio }\end{array}$ & $\begin{array}{c}\text { Assoc } \\
\text { industry }\end{array}$ & Age BP \\
\hline Ly-2370 & BCM-206 & 1979 & 1 & Late Roessen & $5330 \pm 130$ \\
\hline-2326 & BCM-100 & 1978 & $4 / 15$ & Menneville group & $5530 \pm 320$ \\
\hline-2329 & BCM-100 & 1979 & $11 / 15$ & Menneville group & $5100 \pm 160$ \\
\hline-2371 & BCM-202 & 1979 & 1 & Late Roessen & $5340 \pm 130$ \\
\hline .2327 & BCM-124 & 1978 & 1 & Recent Rubané & $6030 \pm 130$ \\
\hline-2328 & CLJ-6-15 & 1977 & $14 / 15$ & Michelsberg & $4810 \pm 120$ \\
\hline-2334 & CCF -359 & 1979 & $11 / 15$ & Michelsberg & $5020 \pm 150$ \\
\hline-1826 & CCF -25 & 1974 & $1 / 4$ & Recent Rubané & $5360 \pm 510$ \\
\hline-1827 & $\mathrm{CCF}-27$ & 1974 & $2 / 5$ & Recent Rubané & $5860 \pm 300$ \\
\hline-1828 & CCF -52 & 1974 & $2 / 5$ & Recent Rubané & $6580 \pm 400$ \\
\hline-1829 & CCF-175 & 1975 & $4 / 5$ & Recent Rubané & $5930 \pm 190$ \\
\hline-1737 & CCF-246 & 1976 & 1 & Recent Rubané & $6220 \pm 230$ \\
\hline-1736 & CCF -230 & 1976 & 1 & Recent Rubané & $6450 \pm 160$ \\
\hline-2321 & CCF -295 & 1978 & $13 / 15$ & Recent Rubané & $5960 \pm 170$ \\
\hline-2330 & CCF $-324-325$ & 1978 & 1 & Recent Rubané & $5910 \pm 130$ \\
\hline-2331 & CCF-311 & 1979 & 1 & Recent Rubané & $6000 \pm 120$ \\
\hline-2332 & CCF -321 & 1979 & $29 / 30$ & Recent Rubané & $5800 \pm 170$ \\
\hline-2333 & $\mathrm{CCF}-357$ & 1979 & 1 & Recent Rubané & $5980 \pm 110$ \\
\hline-2335 & CCF - 378-1 & 1980 & $\hat{l}$ & Recent Rubané & $5840 \pm 140$ \\
\hline-2336 & CCF -375 & 1980 & $7 / 10$ & Recent Rubané & $5960 \pm 150$ \\
\hline-1735 & MDV-13 & 1976 & 1 & Recent Rubané & $6200 \pm 190$ \\
\hline-2324 & MDV-13 & 1976 & 1 & Recent Rubané & $6110 \pm 140$ \\
\hline-2322 & MDV-19 & 1977 & 1 & Recent Rubané & $6030 \pm 190$ \\
\hline-2323 & MDV-39 & 1977 & $7 / 30$ & Recent Rubané & $5860 \pm 190$ \\
\hline-1734 & MDV-1 & 1976 & $4 / 5$ & Recent Rubané & $6140 \pm 210$ \\
\hline-1824 & VSG-70 & 1975 & $5 / 6$ & Neolithic post-Rubané & $6130 \pm 200$ \\
\hline-1825 & VSG-114 & 1976 & $4 / 5$ & Neolithic post-Rubané & $6010 \pm 220$ \\
\hline
\end{tabular}


General Comment (URA 12): disregarding Ly-1826 (much too young) and Ly-1828 and -1736 (too old), this important series of Recent Rubané of Paris Basin sites in Aisne $R$ valley indicates that culture occupied region for ca $300 \mathrm{yr}$ from 6100 to $5800 \mathrm{BP}$. Dates are contemporary with end Linear pottery culture of Rhine and Netherland regions and may be a little later. Dates of Villeneuve-Saint-Germain Neolithic, Ly-1824 and -1825 are contemporary with Recent Rubané, which is surprising because typologic evidence suggests younger age. Both Michelsberg dates agree with expected range. Late Röessen and Menneville group dates are 1st for these cultures in Paris Basin.

\section{Ly-2463. Bois de Refuge, Misy sur Yonne, Seine et} Marne

Bones from Pit C $\left(48^{\circ} 21^{\prime} \mathrm{N}, 3^{\circ} 04^{\prime} \mathrm{E}\right)$. Coll 1971 by C Mordant and J Bontillot; subm 1981 by C Mordant and D Mordant. Assoc with Late Recent Rubané industry (Mordant \& Mordant, 1977). Comment (CM\& $\mathrm{DM})$ : expected date agrees with many others from Aisne $\mathrm{R}$ valley, where same Recent Rubané industry was found (above).

\section{Vallée de la Seine series, Seine et Marne}

Bones from pits and trenches in Middle or Late Neolithic villages in La Seine R valley. Coll 1973 to 1980 by C Mordant and D Mordant; subm by D Mordant. Table 24A lists sites; Table 24B lists samples.

TABLE 24A

Seine Valley sites, Seine et Marne district

\begin{tabular}{llcc}
\hline \multicolumn{1}{c}{ Site } & \multicolumn{1}{c}{ Village } & Loc & Ref \\
\hline Le Gros Bois & Balloy & $\left(48^{\circ} 24^{\prime} \mathrm{N}, 3^{\circ} 09^{\prime} \mathrm{E}\right)$ & Mordant (1967) \\
Maram & Catenay sur Seine & $\left(48^{\circ} 25^{\prime} \mathrm{N}, 3^{\circ} 06^{\prime} \mathrm{E}\right)$ & \\
Chemin de la Tombe & Gravon & $\left(48^{\circ} 24^{\prime} \mathrm{N}, 3^{\circ} 07^{\prime} \mathrm{E}\right)$ & \\
Les Roqueux & Grisy sur Seine & $\left(48^{\circ} 26^{\prime} \mathrm{N}, 3^{\circ} 19^{\prime} \mathrm{E}\right)$ & Mordant \& Mordant \\
Le Haut des Nachères & Noyen sur Seine & $\left(48^{\circ} 26^{\prime} \mathrm{N}, 3^{\circ} 21^{\prime} \mathrm{E}\right)$ & \begin{tabular}{c} 
Mordat7) \\
\hline
\end{tabular} \\
\hline
\end{tabular}

TABLE 24B

Seine Valley samples

\begin{tabular}{cllccccc}
\hline $\begin{array}{c}\text { Lab } \\
\text { no. }\end{array}$ & \multicolumn{1}{c}{ Site } & \multicolumn{1}{c}{ Loc } & $\begin{array}{c}\text { Colln } \\
\text { date }\end{array}$ & $\begin{array}{c}\text { Dilution } \\
\text { ratio }\end{array}$ & Industry & Age BP \\
\hline Ly-2460 & Balloy & Pit 4 & 1965 & 1 & $\begin{array}{l}\text { Neolithic } \\
\text { (middle-late) }\end{array}$ & $4770 \pm 160$ \\
-2459 & Gravon & Pit FA 1 & 1979 & $1 / 5$ & $\begin{array}{l}\text { Neolithic } \\
\text { (middle-late) }\end{array}$ & $4900 \pm 210$ \\
-2457 & Noyen/Seine I & Pit FD 3 & 1973 & 1 & $\begin{array}{l}\text { Neolithic } \\
\text { (middle-late) }\end{array}$ & $4870 \pm 160$ \\
-2458 & Noyen/Seine I & Pit F B & 1973 & $1 / 4$ & $\begin{array}{l}\text { Neolithic } \\
\text { (middle-late) }\end{array}$ & $5260 \pm 200$ \\
-2461 & Noyen/Seine I & Enclosure II & 1979 & $4 / 5$ & $\begin{array}{l}\text { Neolithic } \\
\text { (middle-late) }\end{array}$ & $4970 \pm 140$ \\
-2462 & Noyen/Seine I & ABC I & 1979 & $1 / 3$ & $\begin{array}{l}\text { Neolithic } \\
\text { (middle-late) }\end{array}$ & $5140 \pm 170$ \\
-2456 & Grisy sur Seine & Enclosure F 3 & 1980 & $3 / 10$ & $\begin{array}{l}\text { Neolithic } \\
\text { (middle-late) }\end{array}$ & $5100 \pm 180$ \\
\hline
\end{tabular}


General Comment (CM\&DM): oldest dates are contemporaneous with unpub result for Chassean industry at Joncquières site, Oise, Gif-2919: 5120 \pm 130 . However, all these industries mainly come from $\mathrm{N}$ origin (Michelsberg) and ceramics of some sites are similar to Early Michelsberg style (MK I/II) in most of sites in Rhône Valley from 5500 to 5300 BP. Youngest dates indicate length of human occupation at Noyen site and in situ evolution of ceramic styles which remain contemporary with more recent ceramics of German MK (Mordant \& Poitout, in press).

Ly-2464. Les Chappes, Molay, Yonne

$4460 \pm 110$

Bones from silo pit in Late Neolithic site $\left(47^{\circ} 44^{\prime} \mathrm{N}, 4^{\circ} 56^{\prime} \mathrm{E}\right)$. Coll 1980 and subm 1981 by C Mordant. Comment (CM): date seems a little too young because of similarity of assoc industry with Noyen industry, Ly-4810: $5260 \pm 200$ (above).

\section{Ly-2455. Les Grèves de Frécul, Barbuise-Courtavant, Aube}

$5530 \pm 150$

Bones from Pit 23 of Cerny culture (Middle Neolithic) $\left(48^{\circ} 39^{\prime} \mathrm{N}\right.$, $\left.3^{\circ} 32^{\prime} \mathrm{E}\right)$. Coll 1970 by J Piette and subm 1970 by $\mathrm{D}$ Mordant and J Piette. (2/3 diluted sample). Comment (DM): date confirms site is contemporary with two sites with same Cerny industry previously dated by Gif-5002: $5510 \pm 140$ вP (unpub) at Jablines (Seine et Marne) and Gif5005: $5630 \pm 120$ at Pincevent (Seine et Marne). Cerny culture seems to be homogeneous in E Ile de France region.

\section{Ly-1944. Le Creux-Rouge, Clermont-Ferrand, Puy de Dôme}

Human bones from grave in volcanic ash $\left(45^{\circ} 47^{\prime} \mathrm{N}, 3^{\circ} 5^{\prime} \mathrm{E}\right)$. Coll 1973 and subm 1979 by J P Daugas and J P Raynal. Comment (JPD\& JPR): date agrees with archaeol estimate of grave at Early Middle Neolithic of Auvergne region, $i e$, at limit between Pre-Chassean and Chassean cultures (Daugas \& Malacher, 1978; Raynal \& Daugas, 1979).

\section{Ly-1797. La Chaise, Malesherbes, Loiret $\quad 6190 \pm 210$}

Human bones from under flagstone-covered sepulture $\left(48^{\circ} 17^{\prime} \mathrm{N}, 2^{\circ}\right.$ $23^{\prime}$ E). (3/5 diluted sample). Coll 1978 by J Vintrou and subm 1979 by G Richard, Orléans. Comment (GR): this type of sepulture which surely occurred before dolmens was never dated by industries. Date, oldest for all W central France, makes this monument oldest megalithic monument in France, which is contemporaneous with Recent Rubané culture in Aisne R Valley (above) (Richard \& Vintrou, 1979).

\section{Vieille Eglise series, La Balme de Thuy, Haute-Savoie}

Bones of deer and boar from two levels of La Vieille Eglise rock shelter $\left(45^{\circ} 55^{\prime} \mathrm{N}, 6^{\circ} 17^{\prime} \mathrm{E}\right)$. Coll 1977 and subm 1978 by J P Ginestet, Thones. 
Ly.1934. Level 5A

$5940 \pm 210$

From Layer 5A, assoc with Chassean and Cortaillod ceramics and uncharacteristic flints. (7/9 diluted sample)

\section{Ly.1935. Level 5B}

$6500 \pm 230$

From Layer 5B, assoc with same ceramics as Ly-1934 but lithic industry shows Tardenoisian (Mesolithic) influence. (2/3 diluted sample)

General Comment (JPG): both dates agree with each other and confirm expected ages and fairly old age of Layer 5B with Mesolithic influences.

\section{Ly-2198. Hassi Mouillah, Ouargla, Algeria}

$5660 \pm 210$

Charcoal from level assoc with Neolithic of Capsian tradition in Hassi Mouillah site on side of Mellala Sebkha $\left(31^{\circ} 58^{\prime} \mathrm{N}, 5^{\circ} 22^{\prime} \mathrm{E}\right)$. Coll 1977 by $\mathrm{G}$ Trécolle and subm 1978 by M Schvoerer, Lab Physique Appl Archaeol, Univ Bordeaux. Dated to cross-check dates by TL method. Comment (MS): date agrees fairly well with previous unpub result, Gif438: са 5280 BP, obtained from same level. Three TL dates from upper part of site gave following values: BDX-110: $7890 \pm 680 \mathrm{BP}, \mathrm{BDX}-112$ : $6570 \pm 560 \mathrm{BP}$, and BDX-114: $6270 \pm 540 \mathrm{BP}$. Considering statistical margins of both methods and MASCAA calibration of ${ }^{14} \mathrm{C}$ dates (which established true ages of ca $4400 \mathrm{BC}^{*}$ ), agreement between both methods seems satisfactory.

\section{Mehrgarh series, Baluchistan, Pakistan}

Table 25 lists samples of charcoal from tell near Kachi $\left(29^{\circ} 20^{\prime} \mathrm{N}\right.$, $\left.66^{\circ} 12^{\prime} \mathrm{E}\right)$. Coll by French Archeol Mission Indus and subm by J F Jarrige, Mus Guimet, Paris.

TABLE 25

Mehrgarh

\begin{tabular}{|c|c|c|c|c|c|c|}
\hline $\begin{array}{l}\text { Lab } \\
\text { no. }\end{array}$ & $\begin{array}{l}\text { Sample } \\
\text { no. }\end{array}$ & $\begin{array}{c}\text { Loc } \\
\text { and layer }\end{array}$ & $\begin{array}{l}\text { Assoc } \\
\text { industry }\end{array}$ & $\begin{array}{c}\text { Colln } \\
\mathrm{yr}\end{array}$ & $\begin{array}{l}\text { Dilution } \\
\text { ratio }\end{array}$ & Age BP \\
\hline Ly-1527 & MRI-MRK 2B & Loc $93,-1 \mathrm{~m}$ & Bronze age & 1976 & 1 & $3570 \pm 130$ \\
\hline-1529 & MR2-MRK $9 \mathrm{H}$ & Loc $204,-1.1 \mathrm{~m}$ & Bronze age & 1976 & 1 & $3960 \pm 140$ \\
\hline-1528 & MR3-MRC 8I & Loc ?,$-3.5 \mathrm{~m}$ & Bronze age & 1976 & 1 & $4190 \pm 140$ \\
\hline-1945 & MR4-F5F & Layer $4,-2.2 \mathrm{~m}$ & Chalcolithic & 1978 & $7 / 10$ & $5360 \pm 310$ \\
\hline-1947 & MR-3T 536 & Layer $4,-3.5 \mathrm{~m}$ & Neolithic & 1979 & 1 & $5830 \pm 190$ \\
\hline-1946 & MR3 AIA 433 & $\begin{array}{l}\text { Layer } 3,-1.9 \mathrm{~m} \\
\text { without humus }\end{array}$ & Neolithic & 1979 & 1 & $33,000 \pm 3000$ \\
\hline-1950 & MR3 AIA & $\begin{array}{l}\text { Layer } 3,--1.9 \\
\text { with humic } \\
\text { fraction }\end{array}$ & Neolithic & 1979 & 1 & $8440 \pm 250$ \\
\hline-1949 & MR37537 & $\begin{array}{l}\text { Layer } \mathrm{X}-3.7 \mathrm{~m} \\
\text { only humic } \\
\text { fraction }\end{array}$ & Neolithic & 1979 & 1 & $5530 \pm 180$ \\
\hline-1948 & MR37 537 & $\begin{array}{l}\text { Layer X }-3.7 \mathrm{~m} \\
\text { without humic } \\
\text { fraction }\end{array}$ & Neolithic & 1979 & $1 / 6$ & $5720 \pm 730$ \\
\hline
\end{tabular}


General Comment: as site was probably occupied from 5500 to 3500 BP for Chalcolithic upper layers and са 7500 BP for Neolithic lower layers, dates do not agree with expected ages and are either too young by at least $2000 \mathrm{yr}$, or obviously too old. For last two, Ly-1946 and -1950, old ages can be explained. Charcoal came from hearths where burned wicker baskets were treated with bitumen, which is found near site, at time of manufacture. Thus, dead carbon was introduced to sample and measured later with it. All other dates seem too young but sample cannot have been contaminated by modern organic soluble components as humic fraction, Ly-1949, and no humic fraction, Ly-1948, have same apparent age. Discrepancy with expected ages may be explained either by very large amount of ancient roots in layers, eg, because of deep cultivation at ca 5500 BP during temporary abandonment of site, or by fact that site is much younger than expected (Jarrige \& Lechevalier, 1980). Many other dates from other ${ }^{14} \mathrm{C}$ labs from same site show same range of dates and suggest that revision of archaeol stratigraphic interpretation is necessary.

\section{Ly-2483. Erg-Tihodain, Sahara occidental, Algeria $\quad 6010 \pm 160$}

Ostrich egg fragments from black soil at Neolithic site of Capsian tradition, assoc with flint and human skeletons, on W border of Erg Tihodaïn, between Tassali and Hoggar in central Sahara desert $\left(25^{\circ} 19^{\prime}\right.$ $\left.\mathrm{N}, 6^{\circ} 50^{\prime} \mathrm{E}\right)$. Coll and subm 1980 by A Bonnet, Nîmes. Comment (AB): date agrees with expected value and others of Neolithic Capsian sites (Camps, Delibrias, \& Thommeret, 1968). Result indicates suitability of this dating material. Another date, from $6 \mathrm{~km} \mathrm{~N}$ of site, in black soil with ceramics, was previously pub, Ly-407: $6870 \pm 150(\mathrm{R}, 1973$, v 15, p 146).

\section{Ly-2149. EI Haroua II, Témara, Morocco}

$5900 \pm 210$

Bones from double Neolithic sepulture, Rabat region $\left(33^{\circ} 57^{\prime} \mathrm{N}, 6^{\circ}\right.$ $56^{\prime}$ W). Coll 1978 by A Debénath, Prehist Paleontol Mission Morocco, and subm 1979 by A Debénath. Assoc with Neolithic industry. Comment (AD): date agrees with expected archaeol range; no other absolute dates from seashore of N Morocco (Debénath \& Sbihi-Alaoui, 1979).

\section{F. Mesolithic}

\section{Murchison R series, Australia}

Table 26 lists samples from open-air sites or rock shelters in Murchison R Basin, near Mullewa $\left(27^{\circ} 30^{\prime} \mathrm{S}, 115^{\circ} 00^{\prime} \mathrm{E}\right)$ at Billibilong Spring, Billily Claypan, Inguelba Shelter, Wail Outcamp, and Yallalong Sta, and in Sandford R Valley, near Cue $\left(27^{\circ} 20^{\prime} \mathrm{S}, 117^{\circ} 55^{\prime} \mathrm{E}\right)$ at Walga Rock. Coll by C Dortch, W Australia Mus and F Bordes, J P Raynal, and C Thibault, Inst Quaternaire, Bordeaux, for Fr Archaeol Mission Australia; subm by F Bordes and J P Raynal. 
TABLE 26

Murchison River Basin

\begin{tabular}{|c|c|c|c|c|c|c|}
\hline $\begin{array}{c}\text { Sample } \\
\text { no. }\end{array}$ & $\begin{array}{l}\text { Site } \\
\text { \& ref }\end{array}$ & $\begin{array}{l}\text { Level } \\
\text { depth }\end{array}$ & Sample & $\begin{array}{c}\text { Colln } \\
\text { yr }\end{array}$ & $\begin{array}{l}\text { Dilution } \\
\text { ratio }\end{array}$ & Age BP \\
\hline Ly-1810 & Billibilong 3 & Unit 2 base & Charcoal & $1978 / 1978$ & $1 / 4$ & $2030 \pm 330$ \\
\hline .1809 & Billibilong 2 & Unit 3 base & Charcoal & $1978 / 1978$ & 1 & $3590 \pm 130$ \\
\hline-2169 & Billibilong 8 & Unit 3 base & Charcoal & $1979 / 1980$ & $1 / 4$ & $3810 \pm 130$ \\
\hline-2170 & Billibilong 6 & Unit 4 base & Charcoal & $1979 / 1980$ & $1 / 4$ & $4000 \pm 220$ \\
\hline-2079 & Billily 1 & Unit 2 base & Charcoal & $1979 / 1980$ & 1 & $650 \pm 110$ \\
\hline-2366 & Inguelba 1 & $10-15 \mathrm{~cm}$ & Charcoal & $1980 / 1980$ & $19 / 30$ & $270 \pm 160$ \\
\hline-2367 & Inguelba 2 & $25-30 \mathrm{~cm}$ & Charcoal & $1980 / 1980$ & $1 / 2$ & $560 \pm 160$ \\
\hline-2168 & Wail Outcamp 3 & Unit 4 top & Charcoal & $1979 / 1980$ & 1 & $2420 \pm 120$ \\
\hline-2080 & Wail Outcamp 4 & Unit 4 top & $\begin{array}{c}\text { Calcareous } \\
\text { algae }\end{array}$ & 1979 & 1 & 110 \\
\hline-2081 & Wail Outcamp 7 & Unit 4 top & Shells & $1979 / 1980$ & $1 / 5$ & $4650 \pm 290$ \\
\hline-2167 & Yallalong 5 & Unit 4 base & Shells & $1979 / 1980$ & 1 & $4210 \pm 130$ \\
\hline-2097 & Walga Rock 3 & $70-75 \mathrm{~cm}$ & Charcoal & $1978 / 1979$ & $2 / 3$ & $790 \pm 160$ \\
\hline-2098 & Walga Rock 4 & $57.80 \mathrm{~cm}$ & Charcoal & $1978 / 1979$ & $1 / 3$ & $1040 \pm 180$ \\
\hline-2099 & Walga Rock 6 & $110-115 \mathrm{~cm}$ & Charcoal & $1978 / 1979$ & $2 / 3$ & $3820 \pm 200$ \\
\hline-1846 & Walga Rock 1 & $110-115 \mathrm{~cm}$ & Cha & $1978 / 1978$ & $2 / 5$ & $7010 \pm 350$ \\
\hline-1847 & Walga Rock 2 & $115-125 \mathrm{~cm}$ & Charcoal & $1978 / 1978$ & $1 / 10$ & $9950 \pm 750$ \\
\hline
\end{tabular}

General Comment (JPR): dates establish chronology of recent lithic industries of Murchison R Basin. Period of occupations with non-microlithic industries is seen in Layer 11 of Walga Rock site. This layer is deeply channelled by subsequent humid period. Microlithic industries appear in Units 4 and 2 at Walga Rock, Billibilong, and Billily during semi-arid period with wind deposits, sedimentation, and colluvia. These microlithic industries last up to very recent period at Walga Rock and Ingulba Shelter.

Ly-2365. La Source, Cosnac, Corrèze $\quad \mathbf{7 2 7 0} \pm \mathbf{2 4 0}$

Charcoal from sandstone fill of rock shelter at Roche-Longue $\left(45^{\circ} 08^{\prime}\right.$ $\mathrm{N}, 1^{\circ} 35^{\prime} \mathrm{E}$ ). Coll 1980 by $\mathrm{P}$ Andrieu and P Chennebault, and subm 1980 by $G$ Mazière, Dir Antiquités Préhist, Limoges. (11/30 diluted sample). Comment (GM): date is a little too young for Early or Middle Sauveterrian assoc industry; this may be due to downward infiltration of recent charcoal or rootlets.

\section{Ly-2297. Abri des Cabônes, Ranchot, Jura}

$8730 \pm 170$

Bones from upper layer in Les Cabônes shelter. Coll 1980 by M Campy and S David, and subm 1980 by M Campy, Hist Geol Paleontol Lab, Besançon. Assoc with triangle Mesolithic industry. (3/5 diluted sample). Comment (MC): date agrees with typologic and palynologic attribution to Boreal period. Cf unpub Louvain date from Gigot shelter at Bretonvillers, Doubs, Lv-1112: $8500 \pm 95$ BP.

\section{Ly-2200. Les Mians, Gordes, Vaucluse \\ $8620 \pm 380$}

Charcoal from Les Luquets rock shelter $\left(43^{\circ} 55^{\prime} \mathrm{N}, 5^{\circ} 11^{\prime} \mathrm{E}\right)$. Coll and subm 1979 by $M$ Livache. (1/5 diluted sample); assoc with Sauveterrian industry (Livache, 1976). Comment (ML): date is comparable to results from neighboring site Gramari, ie, with Levels $3 \mathrm{~B}$ and $4 \mathrm{C}$, respec- 
tively, Gif-753: $8000 \pm 190$ and Gif-754: $9340 \pm 220$ (R, 1971, v 13, p 219). It also fits with other dates for this Sauveterrian industry in other regions in France.

\section{La Pécoulette series, Lagorce, Ardèche}

Table 27 lists samples from site lying at entrance to La Pécoulette grotto $\left(44^{\circ} 24^{\prime} \mathrm{N}, 4^{\circ} 19^{\prime} \mathrm{E}\right)$. Coll 1977 and subm 1978 by $\mathrm{D}$ Philibert, Univ Lyon. Assoc with Sauveterrian (Epipaleolithic) industry (Philibert \& Debard, 1977-78).

TABLE 27

La Pécoulette

\begin{tabular}{rlllll}
\hline $\begin{array}{c}\text { Sample } \\
\text { no. }\end{array}$ & \multicolumn{1}{c}{ Square } & Depth & Sample & $\begin{array}{c}\text { Dilution } \\
\text { ratio }\end{array}$ & Age BP \\
\hline Ly-2364 & La Pécoulette A2 & $60-70 \mathrm{~cm}$ & Bones & $7 / 30$ & $8450 \pm 350$ \\
-2410 & La Pécoulette A2 & $70-75 \mathrm{~cm}$ & Charcoal & $1 / 6$ & $6280 \pm 320$ \\
-1978 & La Pécoulette ad & $75-100 \mathrm{~cm}$ & Bones & $7 / 10$ & $8570 \pm 320$ \\
-2411 & La Pécoulette A2 & $100-110 \mathrm{~cm}$ & Bones & $3 / 10$ & $8740 \pm 230$ \\
-1979 & La Pécoulette A2 & $110-120 \mathrm{~cm}$ & Bones & $1 / 5$ & $9060 \pm 800$ \\
-1980 & La Pécoulette A2 & $125-135 \mathrm{~cm}$ & Bones & $2 / 9$ & $8200 \pm 750$ \\
\hline
\end{tabular}

General Comment (DP): single charcoal date is too young probably because of roots and shallow level. Bone dates are in expected range for Epipaleolithic industry. Low collagen content and small sample size made statistical margins too large to check ages stratigraphically.

\section{Ly-2107. La Madeleine des Albis, Penne, Tarn $\quad 8850 \pm 190$}

Bones from $2.5 \mathrm{~m}$ depth in small fissure filled with geol and archaeol sediments close to Magdalenian site (44. $\left.05^{\prime} \mathrm{N}, 1^{\circ} 43^{\prime} \mathrm{E}\right)$. Coll 1977 by $\mathrm{H}$ Bessac and subm 1978 by J Lautier, Albi. No assoc industry but upper part of fissure deposit contains potsherds from Middle ages. Comment $(\mathrm{JL})$ : date shows that bones are either Mesolithic or mixing of recent and other bones from neighboring Magdalenian sites previously dated at Ly-1109: 11,180 \pm 300 and $-1175: 10,110 \pm 440(\mathrm{R}, 1978, \mathrm{v} 20$, p 46-47).

\section{G. Paleolithic}

\section{Ly-1970. Pierre Magnat, Fromental, Haute-} Vienne

Modern $\Delta^{14} \mathrm{C}=\mathbf{1 . 1} \pm \mathbf{2 . 3 \%}$

Charcoal from $60 \mathrm{~cm}$ depth in foot-hill site $\left(46^{\circ} 09^{\prime} \mathrm{N}, 1^{\circ} 27^{\prime} \mathrm{E}\right)$. Coll 1978 by R Crédot and M Dominique, and subm 1979 by G Mazière. Assoc with presumably Late Magdalenian industry. (3/5 diluted sample). Comment (GM): date indicates upper layers of site were re-used or disturbed by recent roots.

\section{Ly-1605. Martinet, Sauveterre-La-Lemance, Lot et Garonne}

Bones from Layer IV in Le Martinet site $\left(44^{\circ} 36^{\prime} \mathrm{N}, 1^{\circ} 01^{\prime} \mathrm{E}\right)$. Coll during ancient excavation by $\mathrm{L}$ Coulonges and subm 1977 by J M Le Tensorer, Univ Bordeaux. Comment (JML): uncertainty margin of date is very large, because of small amount of collagen. Date may only be con- 
sidered compatible with archaeol attribution to Magdalenian (Le Tensorer, 1980; 1981).

\section{Abaunty series, Arrais, Navarre, Spain}

Sample from Abauntz grotto (43 $\left.01^{\prime} \mathrm{N}, 1^{\circ} 42^{\prime} \mathrm{W}\right)$. Coll from 1976 to 1978 and subm 1979 by P Utrilla-Miranda, Univ Zaragoza.

Ly-1964. 19/20

$9530 \pm 300$

Burned bones from Level "d" assoc with Azilian (Epipaleolithic) culture without geometrics. (2/5 diluted sample)

Ly-1965. 19/20

$15,800 \pm 350$

Bones from Level "e" assoc with Early Magdalenian industry without harpoon.

General Comment (PU-M): dates agree with expected ages. Ly-1964 is comparable to dates from Zatoya site at Abaurrea Alta, Navarre, Levels I and II, Ly-1457: $8260 \pm 550$ and Ly-1398: 8150 \pm 170 (R, 1979, v 21, p 442). Ly-1965 is contemporaneous with Altamira site at Santillana del Mar, Santander, M-829: 15,500 \pm 700 (R, 1969, v 11, p 109), with Juyo at Igollo, Santander, M-830: 15,300 \pm 700 (R, 1968, v 10, p 46) or with Pascano series, BM-1455: 16,560 \pm 131, BM-1453: 15,988 \pm 193 , and BM1452: $15,173 \pm 160(\mathrm{R}, 1982$, v 24, p 249-250) (Utrilla-Miranda, in press).

\section{Le Calvaire series, Lourdes, Hautes-Pyrénées}

Bone fragments from two geol secs $\left(43^{\circ} 06^{\prime} \mathrm{N}, 0^{\circ} 07^{\prime} \mathrm{E}\right)$. Coll 1977 and subm 1978 by J Omnès.

\section{Ly-1905. Left sec, level 0/0.30m}

Ly-1906. Front sec, level 1.70/2.50m

$12,450 \pm 330$

General Comment (JO): Ly-1905 corresponds with little known microlithic industry which should be Epipaleolithic. As this is 1st find of its kind in region, date cannot be compared. Ly-1906 corresponds with Late Magdalenian industry and with other dates of region (Clot \& Omnès, 1980) eg, at Espelugues, Ly-1406: 13,170 \pm 260 (R, 1979, v 21, p 444).

\section{Ly-2184. Fontanet Foyer, Ornalac-Ussat-Les- Bains,} Ariège

$12,770 \pm 420$

Charcoal from hearth in Fontanet grotto $\left(42^{\circ} 49^{\prime} \mathrm{N}, 1^{\circ} 38^{\prime} \mathrm{E}\right)$. Coll and subm 1979 by J Cilottes, Dir Antiquités préhist, Foix. (2/11 diluted sample). Comment (JC): archaeol evidence suggests that grotto was occupied for only short periods of time. Date seems to confirm previous measurement, Ly-846: 19,810 $\pm 740(\mathrm{R}, 1975, \mathrm{v} 17, \mathrm{p} 23)$ considering statistical margins of both dates, average of which is Ly-846.2184: 13,020 \pm 370 .

Ly-2296. Abri des Cabônes, Ranchot, Jura

$12,620 \pm 250$

Bones from lower layer of les Cabônes shelter $\left(47^{\circ} 09^{\prime} \mathrm{N}, 5^{\circ} 44^{\prime} \mathrm{E}\right)$. Coll 1980 by M Campy and S David, subm 1980 by M Campy. (13/15 diluted sample). Assoc with Late Magdalenian industry. Comment (MC): correspondence of date with generally accepted range of dates of Bölling 
period is surprising for assoc Late Magdalenian industry in region but agrees with other dates from distant French sites such as Le ChamoisBoivin grotto at Blois/Seille, Ly-440: 12,040 \pm 270 (R, 1973, v 15, p 168).

\section{Enval series, Vic-le-Comte, Puy de Dôme}

Charcoal from Durif shelter $\left(45^{\circ} 29^{\prime} \mathrm{N}, 3^{\circ} 14^{\prime} \mathrm{E}\right)$, from so-called Sol de la Grange part of site. Sample corresponds to Magdalenian industry a little older than that of Fond de l'Abri part of site previously dated, Ly-425: 13,000 $\pm 300(\mathrm{R}, 1973, \mathrm{v} 15, \mathrm{p} 149)$ and Ly-727: 13,700 $\pm 380(\mathrm{R}$, 1975, v 17, p 27). Coll 1973 and subm 1978 by Y Bourdelle, ClermontFerrand (Bourdelle, 1979).

Ly-2046. No. 304

$13,090 \pm 270$

From Level D1. (2/3 diluted sample)

Ly-2047. No. 35

$6440 \pm 350$

From Level B. (1/5 diluted sample)

General Comment (YB): despite expected older age, Ly-2046 indicates same range of date as previous results. Ly-2047 is aberrant and proves local contamination at site.

\section{Comarque series, Sireuil, Dordogne}

Bones from right part of small grotto $\left(44^{\circ} 57^{\prime} \mathrm{N}, 1^{\circ} 06^{\prime} \mathrm{E}\right)$. Coll and subm 1979 by B Delluc and G Delluc, Périgueux. Assoc lithic industry may be defined as Magdalenian but without characteristic elements, and wall of grotto has engravings (Delluc \& Delluc, 1981).

\section{Ly-2154. 1}

$13,370 \pm 340$

Bones of miscellaneous animal spp. (2/3 diluted sample)

Ly-2355. 2

$12,710 \pm 200$

Bones of reindeer.

General Comment (BD\&GD): Ly-2355 confirms -2154 and homogeneity of bone material of grotto deposits. Average of both dates is Ly-2154/2355: $12,880 \pm 170$, agreeing with expected age according to style of engravings (Early Style IV, according to Leroi-Gourhan), assoc industry (Magdalenian III or IV), and vegetation (cold climatic phase indicated by pollen analysis). Occupation time of grotto may have occurred during last part of Early Dryas period.

\section{Moulin Neuf, Saint Quentin de Baron, Gironde}

Bone fragments from rock shelter $\left(44^{\circ} 38^{\prime} \mathrm{N}, 0^{\circ} 16^{\prime} \mathrm{W}\right)$. Coll 1977 and subm 1979 by M Lenoir, Univ Bordeaux. Assoc with Late or Middle Magdalenian industry without harpoons (Lenoir, 1977).

\section{Ly-2352. $\quad \mathrm{Cz}$ a}

$13,570 \pm 260$

From top of Layer 2. (2/3 diluted sample)

Ly-2275. Cz b

From base of Layer 2. (3/5 diluted sample)

$14,280 \pm 440$ 
General Comment (ML): both dates suggest that assoc industry belongs to Middle Magdalenian.

Ly-2100. La Marche, Lussac-les-Chateaux, Vienne $\quad 14,280 \pm 160$

Bones from only layer of La Marche grotto $\left(46^{\circ} 24^{\prime} \mathrm{N}, 0^{\circ} 43^{\prime} \mathrm{E}\right)$. Coll 1957 and subm 1979 by L Pradel, Chatellerault. Assoc with Magdalenian III industry (Pradel, 1958, p 170-191). Comment (LP): date agrees with attribution to Pre-Bölling interstadial deduced from pollen analysis (Leroi-Gourhan, 1973). It is also similar to unpub date from Le Roc au Sorcier shelter, Grn-1913: 14,160 $\pm 100(\mathrm{R}, 1963, \mathrm{v} 5, \mathrm{p} 169)$ and Grn-1903: 13,920 \pm 80 .

\section{Ly-1830. Grotte de la Bergerie, Caniac du Causse, Lot}

$15,830 \pm 400$

Bones from Layer 7 of Paleolithic site at base of grotto, at La Bergerie des Quatre chemins $\left(44^{\circ} 38^{\prime} \mathrm{N}, 1^{\circ} 40^{\prime} \mathrm{E}\right)$. Assoc with atypical poor industry containing pointe à cran flint of Hamburgian type and bone tools (Seronie-Vivien, 1972). Comment (RS-V): date helps classify this poor industry into group of microlithic industries, with or without scalène triangles, of Middle Magdalenian. Such industries came before eponymic Magdalenian (Séronie-Vivien et al, 1979). Date comparable to similar levels at Fongaban, Ly-977: 14,300 \pm 680, Le Flageolet II, Layer IX, Ly918: 15,250 \pm 320, Combe Cullier, Layer IX, Ly-978: 15,030 \pm 330 (R, 1976, v 18, p 80), Ste-Eulalie, Gif-1745: 15,100 \pm 270 and Gif-2194: 15,200 $\pm 300(\mathrm{R}, 1974$, v 16, p 26).

\section{La Grotte Maszycka series, Poland}

Bones from Maszycka grotto near Maszyce, Krakow dist $\left(50^{\circ} 20^{\prime} \mathrm{N}\right.$, $\left.19^{\circ} 40^{\prime} \mathrm{E}\right)$. Coll 1883 by $\mathrm{G}$ Ossowski and subm 1981 by J K Kozłowski, Inst Archaeol, Univ Jagiellonski, Krakow. Assoc with Magdalenian with Navette industry similar to Fr Magdalenien à Navette industry (Kozłowski, 1962).

Ly-2453. Nos. 1 and 2 (incised)

$14,600 \pm 240$

Ly-2454. No. 3, bones

$15,490 \pm 310$

General Comment (JKK): although only incised bones are assoc with Magdalenian culture, both dates close to dates for same industry at Arlay, Jura, Ly-497: 15,320 \pm 370 , Ly-559: 15,770 $\pm 390(\mathrm{R}, 1973$, v 15, p 520), and Le Grand Abri site, La Garenne, Saint Marcel, Indre, C-578: 15,847 \pm 1220 (Libby, 1952).

\section{Ly-1998. Abri de la Chaire à Calvin, Mouthiers, Charente}

$15,440 \pm 440$

Bone fragments from older Magdalenian Layer ( $54^{\circ} 33^{\prime} \mathrm{N}, 0^{\circ} 07^{\prime} \mathrm{E}$ ). Coll 1969 and subm 1979 by J M Bouvier. Inst Quaternaire, Univ Bordeaux I. (5/6 diluted sample). Comment (JMB): date is older than expected and does not agree with Magdalenian facies without harpoons (Bouvier, 1969). It agrees with two unpub dates from Saint-Germain-La- 
Rivière site, Gironde, with similar industries, Gif-5478: 15,300 \pm 410 from Layer C2, and Gif-5479: 16,200 \pm 600 from Layer C4.

Ly-2228. La Tannerie, Lussac-les-Chateaux, Vienne $18,020 \pm 270$

Bones from terrace in front of La Tannerie Grotto $\left(46^{\circ} 24^{\prime} \mathrm{N}, 0^{\circ} 43^{\prime}\right.$ E). Coll 1950 and subm 1980 by L Pradel, Chatellerault. Assoc with Late Solutrean industry (Pradel, 1950). Comment (LP): dates agree with those of Zero Magdalenian industry from Layer 6, Abri Fritch site in same region, Ly-1124: $1124 \pm 350$ вP (R, 1978, v 20, p 50).

\section{Grotte Pégourié series, Caniac du Causse, Lot}

Table 28 lists samples of bones from Pégourié grotto $\left(43^{\circ} 37^{\prime} \mathrm{N}, 1^{\circ}\right.$ $39^{\prime}$ E). Coll and subm by R Séronie-Vivien, Le Bouscat, Gironde.

TABLE 28

Grotte Pégourié

\begin{tabular}{|c|c|c|c|c|c|c|}
\hline $\begin{array}{l}\text { Lab } \\
\text { no. }\end{array}$ & Layer & $\begin{array}{c}\text { Sample } \\
\text { no. }\end{array}$ & $\begin{array}{c}\text { Assoc } \\
\text { industry }\end{array}$ & $\begin{array}{l}\text { Colln } \\
\text { date }\end{array}$ & $\begin{array}{l}\text { Dilution } \\
\text { ratio }\end{array}$ & Age $\mathbf{B P}$ \\
\hline Ly-1390 & 4 & 1209 & Azilian & 1976 & $2 / 3$ & $11,290 \pm 320$ \\
\hline-1598 & 5 top & 1210 & Azilian & 1976 & $4 / 5$ & $13,980 \pm 510$ \\
\hline-1832 & 5 & 646 & Azilian & 1977 & 1 & $11,870 \pm 290$ \\
\hline-1391 & 5 middle & 1211 & Azilian & 1976 & $2 / 3$ & $11,680 \pm 330$ \\
\hline-1833 & 5 & 645 & Azilian & 1977 & 1 & $11,850 \pm 280$ \\
\hline-1392 & 5 base & 1212 & Azilian & 1976 & $1 / 3$ & $12,690 \pm 530$ \\
\hline-1393 & 6 top & 1213 & Azilian & 1976 & $1 / 6$ & $8730 \pm 890$ \\
\hline-1834 & $8 \mathrm{a}$ & 644 & $\begin{array}{l}\text { Early Magdalenian } \\
\dot{a} \text { raclettes }\end{array}$ & 1977 & 1 & $17,400 \pm 460$ \\
\hline-1394 & $8 \mathrm{~b}$ & 1220 & Early Magdalenian & 1Jו & & \\
\hline-1836 & 9 & & $a$ raclettes & 1976 & $1 / 2$ & $17,490 \pm 520$ \\
\hline-1830 & $9 \mathrm{a}$ & 642 & $\begin{array}{l}\text { Early Magdalenian } \\
\dot{a} \text { raclettes }\end{array}$ & 1977 & $9 / 10$ & $17,420 \pm 390$ \\
\hline-1835 & $9 \mathrm{~b}$ & 643 & $\begin{array}{l}\text { Early Magdalenian } \\
\dot{a} \text { raclettes }\end{array}$ & 1977 & $11 / 20$ & $24,200 \pm 1100$ \\
\hline
\end{tabular}

General Comment (RS-V): dates agree with expected ranges of both industries (Séronie-Vivien et al, 1979). As expected, Azilian industry occurred ca 11,500 BP, as in other regions, eg, Alsace at Rochedane, Ly-1192: $11,090 \pm 200(\mathrm{R}, 1978, \mathrm{v} 20, \mathrm{p} 46)$ and Languedoc at Saint Remèze, Ly320: $11,500 \pm 380(\mathrm{R}, 1971, \mathrm{v} 13$, p 62). Early Magdalenian dates are also homogeneous ca 17,400, in agreement with those from Abri Pataud, 4 dates from 16,500 to $1800 \mathrm{BP}(\mathrm{R}, 1978$, v 20, p 50) and at Laugerie Haute, Ly-972: 18,260 $\pm 360(\mathrm{R}, 1976$, v 18, p 80). Ly-1835 was obtained from bones at base of grotto deposit that may belong to another depositional cycle (see Bos grotto, above). Ly-1393 may be compared with Ly-1837 and -1838 (R, 1980, v 22, p 547) and Gif-2568: 8450 \pm 250 (unpub). These four dates, ca $8500 \mathrm{BP}$, are younger than expected and may indicate re-use assoc with climatic phenomena.

\section{Las Caldas series, San-Juan-de-Prioro, Spain}

Table 29 lists samples of bones from three loci in Las Caldos grotto Prov Oviedo $\left(43^{\circ} 20^{\prime} \mathrm{N}, 5^{\circ} 59^{\prime} \mathrm{W}\right)$. Coll 1980 by M S Corchon and subm 1980 by F J Cerda, Prehist Dept, Univ Salamanca. 
TABLE 29

Las Caldas

\begin{tabular}{|c|c|c|c|c|c|}
\hline $\begin{array}{c}\text { Sample } \\
\text { no. }\end{array}$ & Ref & Area & Level & Industry & Age \\
\hline Ly-2427 & C II & Sala II & III-IV & Middle Magdalenian & $13,400 \pm 150$ \\
\hline-2421 & CI 1 & El Pasillo & 3 & Upper Solutrean & $18,250 \pm 300$ \\
\hline-2422 & CI 2 & El Pasillo & 4 & Upper Solutrean & $17,050 \pm 290$ \\
\hline-2423 & CI 5 & El Pasillo & 7 & $\begin{array}{l}\text { Typical Upper } \\
\text { Solutrean }\end{array}$ & $18,310 \pm 260$ \\
\hline-2424 & CI 6 & El Pasillo & 9 & $\begin{array}{l}\text { Typical Upper } \\
\text { Solutrean }\end{array}$ & $19,390 \pm 260$ \\
\hline-2425 & CI 12 & El Pasillo & 12 top & Middle Solutrean & $19,030 \pm 320$ \\
\hline-2426 & CI 9 & El Pasillo & 12 base & Middle Solutrean & $19,480 \pm 260$ \\
\hline-2428 & CE 15 & Sala I & 16 & Middle Solutrean & $19,510 \pm 330$ \\
\hline-2429 & CE 16 & Sala I & 18 & Middle Solutrean & $19,000 \pm 280$ \\
\hline
\end{tabular}

General Comment (FJC): dates agree with each other and with expected ages. They confirm correlations among three excavated secs are comparable to other dates from Spain or SW France. Ly-2428 corresponds to wet and cold climatic phase, attributed to Early Dryas, and agrees with other dates of Middle Magdalenian in France. Two dates of Late Solutrean agree with others of same industry from Chufin site, obtained but unpub by Madrid lab, CSIC-258: 17,420 \pm 200 . Their apparent inversion may be due to post-sedimentary inversion. All other results are consistent with two series from Oullins and Solutré sites, France. They confirm archaeol hypothesis of in situ evolution of Middle Solutrean industry in Layer 12 corresponding to Würmian III-IV interstadial phase, into typical Solutrean in Layers 9 and 7, corresponding to beginning of Early Dryas phase.

Ly-2279. Puy-Jarrige II, Brive-La-Gaillarde, Corrèze $19,310 \pm 790$

Bones from Sq LVI of Rockshelter II (45 $\left.17^{\prime} \mathrm{N}, 1^{\circ} 28^{\prime} \mathrm{E}\right)$. Coll and subm by $\mathrm{G}$ Mazière, Dir Antiquités Préhist, Limoges. (1/6 diluted sample). Comment (GM): date is too young for assoc industry (Perigordian IV with Pointes de Bayac generally dated ca 24,000 BP. Contamination may be due to Middle-age occupation of rockshelter.

\section{Oullins series, La Bastide de Virac, Ardèche}

Bones from La Baume d'Oullins site $\left(44^{\circ} 20^{\prime} \mathrm{N}, 4^{\circ} 32^{\prime} \mathrm{E}\right)$. Coll and subm 1978 by F Bazile, Vauvert. Assoc with à pointes à crans Solutrean industry (Bazile \& Bazile-Robert, 1981). (4/5 diluted sample)

$$
\begin{array}{lll}
\text { Ly-1984. } & \text { Level } D & \mathbf{2 0 , 1 0 0} \pm 500 \\
\text { Ly-1983. } & \text { Level } 9 & \mathbf{2 0 , 0 6 0} \pm 450
\end{array}
$$

General Comment (FB): dates agree with expected ages of assoc industry in $\mathrm{W}$ Languedoc region. They disagree with two dates previously pub (R, 1975, v 17, p 22) and obviously too young, from two Early Solutrean layers in same site, Ly-779: 19,710 \pm 400 for Layer 7 and Ly-798: 19,360 \pm 420 for Layer 6. They agree with 5 dates ca 19,000 BP for Early Salpétrian (Early Magdalenian) industries and unpub Monaco Lab result from Early or Middle Solutrean industry, la Salpêtrière site at Remoulins 
Gard, MC-2449: 21,600 \pm 70 . They also agree with Groningen series for same industry from Laugeric Haute site, Dordogne (R, 1967, v 9, p 116).

\section{Ly-2101. Laraux, Lussac-les-Châteaux, Vienne}

$21,950 \pm 350$

Bones from Layer 3 in Laraux shelter $\left(46^{\circ} 24^{\prime} \mathrm{N}, 0^{\circ} 43^{\prime} \mathrm{E}\right)$ (Pradel, 1979). Coll 1949 and subm 1981 by L Pradel. (29/30 diluted sample). Comment (LP): previous date from same layer, Ly-1739: 21,530 \pm 910 (R, 1979, v 21, p 447). New measurement was made on larger sample which reduces uncertainty margin; average value of both measurements, Ly-1739/2101: 21,890 \pm 330 .

\section{Ly-1863. La Mère Clochette, Rochefort-sur-Nenon, Jura}

Fragments of mammoth tusk from La Mère Clochette grotto $\left(47^{\circ} 9^{\prime}\right.$ $\mathrm{N}, 5^{\circ} 55^{\prime} \mathrm{E}$ ). Coll 1906 by J Feuvrier and subm 1979 by R Desbrosse, Blanzy. Comment (RD): collections of artifacts confirm existence of Mousterian, Aurignacian (points with split base) and Early Perigordian (Châtelperron and Les Cottés points) (Desbrosse, 1981). Ly-1863 indicates much younger age.

\section{Ly-1861. Pech Merle, Cabrerets, Lot}

$11,200 \pm 800$

Charcoal from Excavation VII under so-called La Frise noire painting in Pech Merle grotto $\left(44^{\circ} 31^{\prime} \mathrm{N}, 1^{\circ} 38^{\prime} \mathrm{E}\right.$ ). Coll and subm 1978 by $\mathrm{M}$ Lorblanchet, Centre Natl Recherche Sci, Gramat. (1/15 diluted sample). Parietal painting of grotto belongs to Magdalenian style (ca 15,000 BP). Comment (ML): even considering very large statistical margin, due to small sample size, date is much younger than expected and seems too recent to mark last occupation time of grotto which is surely contemporaneous with painting. Slight contamination by recent carbon may be responsible for this discrepancy.

\section{Abri Moula series, Ardèche}

Table 31 lists samples of bones from shelter deposit near Soyons $\left(44^{\circ}\right.$ $\left.52^{\prime} \mathrm{N}, 4^{\circ} 50^{\prime} \mathrm{E}\right)$. Coll by Crouzet Archaeol Club and subm by P Payen, Valence. Assoc with scattered, presumed Late Mousterian industry.

\begin{tabular}{cllccc}
\multicolumn{7}{c}{ TABLE 31 } \\
Abri Moula \\
\hline $\begin{array}{c}\text { Sample } \\
\text { no. }\end{array}$ & \multicolumn{1}{c}{ Square } & Depth & $\begin{array}{c}\text { Colln } \\
\text { date }\end{array}$ & $\begin{array}{c}\text { Dilution } \\
\text { ratio }\end{array}$ & Age BP \\
\hline Ly-1595 & F2,F3 & $0.7-2 \mathrm{~m}$ & 1976 & 1 & $24,400+2000$ \\
-2217 & F3,F4,G3,G4 & $3.2-3.9 \mathrm{~m}$ & 1980 & 1 & $20,100 \pm 3100$ \\
-2488 & F4,F5,G4,G5 & $4.1 \mathrm{~m}$ & 1980 & 1 & $33,200 \pm 1500$ \\
\hline
\end{tabular}

General Comment (PP): only Ly-2488 agrees with expected age, relatively recent for end of Mousterian industries, which are, thus, contemporaneous with 1st early Late Paleolithic industries, as previously found in other sites; see, eg, Level VIII, Gigny/Suran, Ly-566: 29,500 \pm 1400 (R, 1973, v 15, p 521) and Ly-789: 28,500 $\pm 1400(\mathrm{R}, 1975, \mathrm{v} 17, \mathrm{p} 83)$. Two other 
dates are too young and suggest re-use in upper levels where Mousterian flints may have mixed with younger bones as stratigraphy and geol hist of sediments seem to be fairly complex.

\section{Saint-Cesaire series, Charente-Maritime}

Bones from La Roche à Pierrot site $\left(45^{\circ} 45^{\prime} \mathrm{N}, 0^{\circ} 31^{\prime} \mathrm{W}\right)$. Coll and subm 1979 by $\mathrm{F}$ Lévêque, Dir Antiquités Préhist Poitiers (Lévêque \& Vandermeersch, 1980).

\section{Ly-2192. Ejo sup}

$21,100 \pm 540$

From so-called ensemble jaune-orange upper layer, assoc with ProtoAurignacian industry. (13/30 diluted sample)

\section{Ly-2193. Ejop sup}

$22,960 \pm 840$

From so-called ensemble jaune-orange-pâle upper layer, assoc with Castelperronian (Early Late Paleolithic) industry. (7/30 diluted sample) General Comment (FL): both dates are much too young despite good sample. Large amount of recent organic matter deposited for short time on site before excavation may have contaminated small amount of collagen remaining on bones.

Ly-1793. Esquicho-Grapaou Sainte-Anastasie, Gard 27,700 \pm 1100

Bone from Layer CC2, Rassan (43 $\left.56^{\prime} \mathrm{N}, 4^{\circ} 19^{\prime} \mathrm{E}\right)$. Coll and subm 1978 by $\mathrm{F}$ Bazile, Vauvert. Assoc with Mousterian industry which is last such level in site and may be attributed to Würm-II/III interstadial. (5/6 diluted sample). Comment (FB): age is younger than expected and conflicts with unpub date from Late Paleolithic level overlying SLC1b: MC-2161: $34,540 \pm 2000$ вP.

\section{Grotte Tournal series, Bize-Minervois, Aude}

Table 30 lists samples from several loci in Tournal grotto $\left(43^{\circ} 20^{\prime} \mathrm{N}\right.$, $2^{\circ} 31^{\prime}$ E). Coll and subm by A Tavoso, Univ Marseille. Site is grotto deposition on stalagmitic floors, constituted by very concreted sediments containing Mousterian, Late Aurignacian, and Magdalenian industries. Sediments have been disturbed by suction action of karstic system sometimes open under stalagmitic floors.

TABLE 30

Tournal Grotto

\begin{tabular}{|c|c|c|c|c|c|c|c|}
\hline $\begin{array}{l}\text { Lab } \\
\text { no. }\end{array}$ & $\begin{array}{c}\text { Sample } \\
\text { no. }\end{array}$ & $\begin{array}{c}\text { Layer } \\
\text { and sq }\end{array}$ & Sample & $\begin{array}{l}\text { Assoc } \\
\text { industry }\end{array}$ & $\begin{array}{c}\text { Subm } \\
\text { date }\end{array}$ & $\begin{array}{l}\text { Dilution } \\
\text { ratio }\end{array}$ & Age $\mathbf{B P}$ \\
\hline Ly-1231 & Bize 2 & K31 C & Charcoal & Magdalenian IV & 1975 & 1 & $12,550 \pm 210$ \\
\hline-1894 & Bize 6 & $\mathrm{~K} 31 \mathrm{C}$ & Bone & Magdalenian IV & 1978 & l & $12,860 \pm 320$ \\
\hline-1897 & Bize 8 & $030 \mathrm{I}$ & Bone & Magdalenian & 1979 & 1 & $13,790 \pm 420$ \\
\hline-1232 & Bize 3 & M34 ALM & Bone & Magdalenian & 1975 & $1 / 2$ & $14,530 \pm 510$ \\
\hline-1675 & Bize 4 & M34 AF & Charcoal & Magdalenian & 1975 & $2 / 5$ & $14,770 \pm 970$ \\
\hline-1896 & Bize 7 & N32 PC & Bone & Late Paleolithic & 1978 & 1 & $25,870 \pm 830$ \\
\hline-1895 & Bize 5 & LM32 F & Charcoal & $\begin{array}{l}\text { Early } \\
\quad \text { Aurignacian }\end{array}$ & 1978 & $1 / 6$ & $\geqslant 29.000$ \\
\hline-1031 & Bize 1 & L31 C & $\begin{array}{l}\text { Carbonaceous } \\
\text { earth }\end{array}$ & $\begin{array}{l}\text { Early } \\
\text { Aurignacian }\end{array}$ & 1974 & $1 / 3$ & $\geqslant 34,200$ \\
\hline-1676 & Bize 5 & $\mathrm{~K} 29 \mathrm{C}$ & Charcoal & Mousterian & 1978 & 1 & $33,600 \pm 1300$ \\
\hline-1898 & Bize 9 & P31 G & Charcoal & Mousterian & 1978 & $1 / 4$ & $\geqslant 35,800$ \\
\hline
\end{tabular}


General Comment (AT): because of fractioning of deposits in isolated units, most measurements were made to control correlation assumed from one unit to another. Most dates were expected. Magdalenian occupation of site took place during Middle Magdalenian and beginning of Late Magdalenian epochs although assoc industry is not characteristic. Because of small amount of available Early Aurignacian material, this industry could not be dated (Ly-1895). Ly-1031 seems too old and -1676 too young, with regard to stratigraphy. $\mathrm{U} / \mathrm{Th}$ measurements were made on teeth and bones but dates are different for both samples.

\section{Brugas series, Layer 4, Vallabrix, Gard}

Charcoal from base of rock shelter $\left(44^{\circ} 03^{\prime} \mathrm{N}, 4^{\circ} 29^{\prime} \mathrm{E}\right)$. Coll and subm 1978 by L Meignen, Centre Recherches Archéol, Valbonne. (17/30 diluted sample)

Ly-2038. 1

$\geqslant 32,000$

Ly-2351. 2

$29,000 \pm 860$

General Comment (LM): Ly-2351 is too young for unknown reason. Ly2038 confirms industry does not belong to very Late Mousterian.

\section{HYDROGEOLOGIC SAMPLES}

\section{Wassia series, Saudi Arabia}

Table 32 lists samples of water from five superimposed aquifers of several underground areas of Arabia. Coll from 1974 to 1977 by Bur Recherches Geol Min (BRGM) for Water and Agric Agency Arabian Kingdom. Subm from 1975 to 1977. Aquifers lie in sedimentary basin mainly deposited from Cretaceous to Neogene eras. They are, from lowermost to uppermost: Behiad (from ante-Cenomanian strata), Wassia (from Cenomanian), Um er Radhuma (Paleocene), Alat Kobar, and Neogene. Study was pub in BRGM rept (1980).

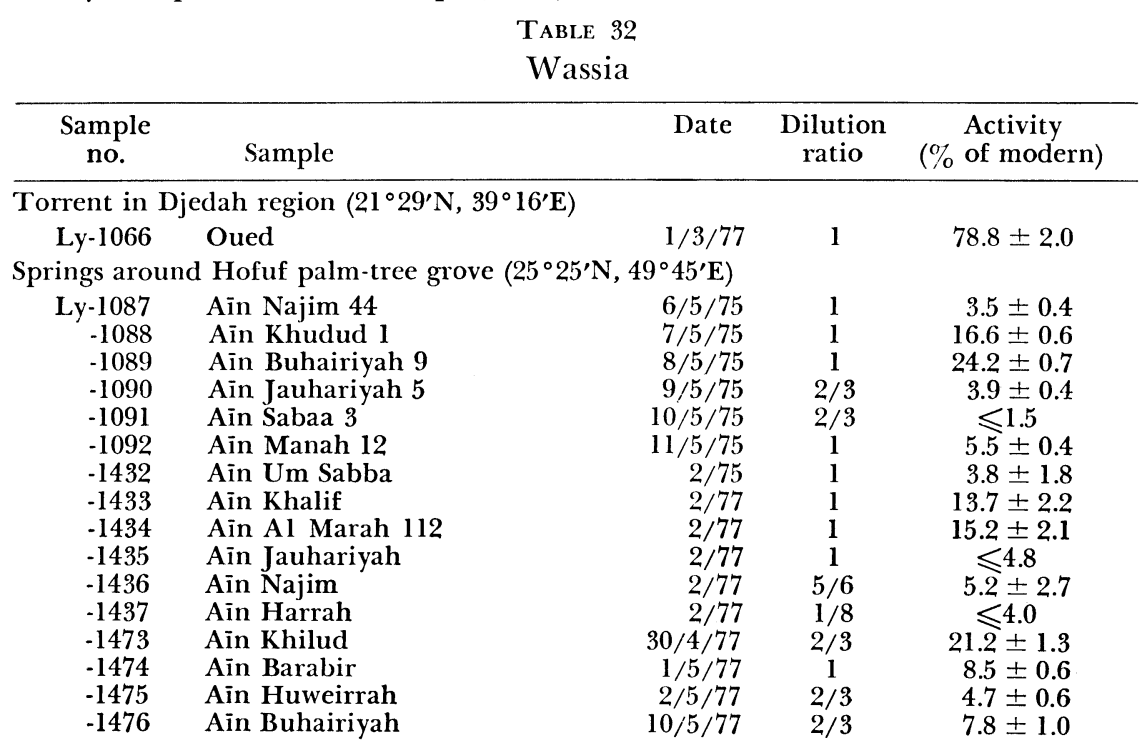


TABLE 32 (continued)

\begin{tabular}{|c|c|c|c|c|c|}
\hline $\begin{array}{l}\text { Sample: } \\
\text { no. }\end{array}$ & \multicolumn{2}{|l|}{ Sample } & Date & $\begin{array}{l}\text { Dilution } \\
\text { ratio }\end{array}$ & $\begin{array}{c}\text { Activity } \\
\text { (\% of modern) }\end{array}$ \\
\hline \multicolumn{6}{|c|}{ Borings in Al Hassa and Hofuf region $\left(25^{\circ} 25^{\prime} \mathrm{N}, 49^{\circ} 45^{\prime} \mathrm{E}\right)$} \\
\hline Ly-1045 & WM 541 & Neogene & $15 / 3 / 75$ & 1 & $9.6 \pm 1.5$ \\
\hline-1048 & WM 194 & Neogene & $18 / 3 / 75$ & 1 & $1.9 \pm 0.3$ \\
\hline-1049 & D 1072 & Neogene & $19 / 3 / 75$ & 1 & $1.5 \pm 0.3$ \\
\hline-1356 & A 1681 & Neogene & 76 & $2 / 3$ & $\leqslant 2.5$ \\
\hline-1360 & G 241 & Neogene & 76 & $2 / 3$ & $\leqslant 2.5$ \\
\hline-1364 & G 001 & Neogene & 76 & $1 / 2$ & $\leqslant 3.0$ \\
\hline-1429 & A 1699 & Neogene & $2 / 77$ & 1 & $16.9 \pm 0.6$ \\
\hline-144 & A 609 & Neogene & $3 / 77$ & $2 / 3$ & $9.7 \pm 1.0$ \\
\hline-1446 & A 818 & Neogene & $3 / 77$ & $5 / 6$ & $\leqslant 1.8$ \\
\hline-1449 & HD $4 \mathrm{~N}$ & Neogene & $3 / 77$ & 1 & $\leqslant 1.9$ \\
\hline-1450 & $\mathrm{HH} 1 \mathrm{~N}$ & Neogene & $3 / 77$ & 1 & $2.1 \pm 0.7$ \\
\hline-1453 & $\mathrm{HH} 2 \mathrm{~N}$ & Neogene & $3 / 77$ & $5 / 6$ & $\leqslant 2.8$ \\
\hline-1455 & $\mathrm{HH} 2 \mathrm{~N}$ & Neogene & $3 / 77$ & 1 & $\leqslant 4.3$ \\
\hline-1456 & HC $6 \mathrm{~N}$ & Neogene & $3 / 77$ & $5 / 6$ & $2.0 \pm 0.6$ \\
\hline-1479 & HC $4 \mathrm{~N}$ & Neogene & $25 / 4 / 77$ & $2 / 3$ & $6.7 \pm 0.8$ \\
\hline$-148 \|$ & HE $2 \mathrm{~N}$ & Neogene & $20 / 4 / 77$ & 1 & $2.7 \pm 0.5$ \\
\hline-1483 & HD $2 \mathrm{~N}$ & Neogene & $8 / 4 / 77$ & $2 / 3$ & $\leqslant 1.5$ \\
\hline-1485 & $\mathrm{HC} 3 \mathrm{~N}$ & Neogene & $4 / 4 / 77$ & $2 / 3$ & $3.8 \pm 0.7$ \\
\hline-1486 & $\mathrm{HC} 2 \mathrm{~N}$ & Neogene & $1 / 5 / 77$ & $2 / 3$ & $6.1 \pm 0.8$ \\
\hline-1487 & HD $1 \mathrm{~N}$ & Neogene & $3 / 5 / 77$ & $2 / 3$ & $2.3 \pm 0.6$ \\
\hline-1492 & HD $5 \mathrm{~N}$ & Neogene & $20 / 5 / 77$ & $2 / 3$ & $3.3 \pm 0.8$ \\
\hline-1500 & HE $1 \mathrm{~N}$ & Neogene & $18 / 6 / 77$ & $2 / 3$ & $\leqslant 2.1$ \\
\hline-1068 & UW 810 & Alat Kobar & $26 / 3 / 75$ & 1 & $3.0 \pm 0.5$ \\
\hline-1047 & WW 810 & Alat Kobar & $16 / 3 / 75$ & 1 & $\leqslant 1.0$ \\
\hline-1352 & B 84 & Alat Kobar & 76 & $1 / 2$ & $\leqslant 2.5$ \\
\hline-1355 & A 613 & Alat Kobar & 76 & $1 / 2$ & $3.6 \pm 1.6$ \\
\hline-1358 & B 85 & Alat Kobar & 76 & $2 / 3$ & $\leqslant 2.5$ \\
\hline-1359 & A 576 & Alat Kobar & 76 & 1 & $\leqslant 2.0$ \\
\hline$-136 \%$ & A 612 & Alat Kobar & 76 & $1 / 2$ & $3.7 \pm 1.2$ \\
\hline-1363 & A 560 & Alat Kobar & 76 & 1 & $\leqslant \overline{3.5}$ \\
\hline-1365 & G 240 & Alat Kobar & 76 & $2 / 3$ & $\leqslant 3.0$ \\
\hline-1428 & $\mathrm{DH} / \mathrm{WW} 2$ & Alat Kobar & $2 / 77$ & $2 / 3$ & $2.8 \pm 0.7$ \\
\hline-1439 & A 570 & Alat Kobar & $3 / 77$ & $2 / 3$ & $\leqslant 2.0$ \\
\hline-1440 & G 013 & Alat Kobar & $3 / 77$ & $2 / 3$ & $\leqslant 2.5$ \\
\hline$-144 \|$ & A 604 & Alat Kobar & $3 / 77$ & $2 / 3$ & $4.1 \pm 0.8$ \\
\hline-1442 & A 1679 & Alat Kobar & $3 / 77$ & 1 & $\leqslant 2.2$ \\
\hline-1444 & U 897 & Alat Kobar & $3 / 77$ & $2 / 3$ & $\leqslant 1.9$ \\
\hline-1452 & $\mathrm{HH} 2 \mathrm{~K}$ & Alat Kobar & $3 / 77$ & $1 / 2$ & $\leqslant 4.0$ \\
\hline-1454 & HC $6 \mathrm{~K}$ & Alat Kobar & $3 / 77$ & 1 & $\leqslant 2.1$ \\
\hline-1478 & $\mathrm{HE} 2 \mathrm{~K}$ & Alat Kobar & $17 / 4 / 77$ & 1 & $2.7 \pm 0.4$ \\
\hline-1480 & $\mathrm{HC} 4 \mathrm{~K}$ & Alat Kobar & $23 / 4 / 77$ & 1 & $6.4 \pm 0.9$ \\
\hline-1490 & HD $3 \mathrm{~K}$ & Alat Kobar & $5 / 5 / 77$ & $2 / 3$ & $2.2 \pm 0.3$ \\
\hline-1498 & HU $5 \mathrm{~K}$ & Alat Kobar & $9 / 6 / 77$ & $2 / 3$ & $4.5 \pm 0.7$ \\
\hline-1503 & HE $1 \mathrm{~K}$ & Alat Kobar & $4 / 7 / 77$ & 1 & $\leqslant 1.6$ \\
\hline-1046 & UW 909 & Um er Radhuma & $16 / 3 / 75$ & 1 & $10.0 \pm 1.5$ \\
\hline-1067 & UW 819 & Um er Radhuma & $25 / 3 / 75$ & $1 / 2$ & $\leqslant 2.0$ \\
\hline-1069 & UW 816 & Um er Radhuma & $27 / 3 / 75$ & 1 & $1.4 \pm 0.3$ \\
\hline-1071 & UW 999 & Um er Radhuma & $29 / 3 / 75$ & $1 / 3$ & $7.2 \pm 1.4$ \\
\hline-1118 & WA 1597b & Um er Radhuma & $4 / 6 / 75$ & $5 / 6$ & $60.7 \pm 1.7$ \\
\hline-1353 & A 596 & Um er Radhuma & 76 & $1 / 3$ & $\leqslant 2.5$ \\
\hline-1354 & S 57 & Um er Radhuma & 76 & 1 & $3.1 \pm 0.7$ \\
\hline$-135^{\prime}$ & SH 808 & Um er Radhuma & 76 & 1 & $\leqslant 2.5$ \\
\hline-1366 & A 828 & Um er Radhuma & 76 & 1 & $2.2 \pm 0.7$ \\
\hline-1423 & U 817 & Um er Radhuma & $2 / 77$ & 1 & $\leqslant 2.5$ \\
\hline-1424 & U 818 & Um er Radhuma & $2 / 77$ & 1 & $4.3 \pm 1.8$ \\
\hline-1425 & AD 812 & Um er Radhuma & $2 / 77$ & 1 & $2.4 \pm 1.6$ \\
\hline-1426 & AD 809 & Um er Radhuma & $2 / 77$ & 1 & $4.5 \pm 2.1$ \\
\hline-1427 & SH 812 & Um er Radhuma & $2 / 77$ & 1 & $\leqslant 2.5$ \\
\hline-1430 & SH 805 & Um er Radhuma & $2 / 77$ & $5 / 6$ & $\leqslant 2.4$ \\
\hline-1431 & HA 814 & Um er Radhuma & $2 / 77$ & 1 & $6.0 \pm 1.8$ \\
\hline-1438 & A 579 & Um er Radhuma & $3 / 77$ & $3 / 5$ & $65.0 \pm 3.5$ \\
\hline-1443 & A 608 & Um er Radhuma & $3 / 77$ & $2 / 3$ & $\leqslant 2.2$ \\
\hline
\end{tabular}


TABLE 32 (continued)

\begin{tabular}{|c|c|c|c|c|c|}
\hline $\begin{array}{l}\text { Sample } \\
\text { no. }\end{array}$ & Sample & & Date & $\begin{array}{l}\text { Dilution } \\
\text { ratio }\end{array}$ & $\begin{array}{c}\text { Activity } \\
\text { (\% of modern) }\end{array}$ \\
\hline $\begin{array}{r}\text { Ly-1447 } \\
-1448 \\
-1451 \\
-1482 \\
-1484 \\
-1488 \\
-1489 \\
-1491 \\
-1499 \\
-1501 \\
-1502 \\
-1070 \\
-1077 \\
-1086 \\
-1477\end{array}$ & $\begin{array}{l}\text { A 562b } \\
\text { HD 4 U } \\
\text { HH 1 U } \\
\text { HH } 5 \text { U } \\
\text { HD 2 U } \\
\text { HD 1 U } \\
\text { U 904 } \\
\text { HD } 5 \text { U } \\
\text { HC } 5 \text { U } \\
\text { HH } 3 \text { U } \\
\text { HE 1 U } \\
\text { UW } 887 \\
\text { HA 1 W } \\
\text { HA 2 W } \\
\text { HD } 4 \text { TW }\end{array}$ & $\begin{array}{l}\text { Um er Radhuma } \\
\text { Um er Radhuma } \\
\text { Um er Radhuma } \\
\text { Um er Radhuma } \\
\text { Um er Radhuma } \\
\text { Um er Radhuma } \\
\text { Um er Radhuma } \\
\text { Um er Radhuma } \\
\text { Um er Radhuma } \\
\text { Um er Radhuma } \\
\text { Um er Radhuma } \\
\text { Wassia or Behjar } \\
\text { Wassia or Behjar } \\
\text { Wassia or Behjar } \\
\text { Wassia or Behjar }\end{array}$ & $\begin{array}{r}3 / 77 \\
25 / 3 / 77 \\
3 / 77 \\
27 / 4 / 77 \\
7 / 4 / 77 \\
2 / 5 / 77 \\
4 / 5 / 77 \\
20 / 5 / 77 \\
30 / 5 / 77 \\
4 / 7 / 77 \\
4 / 7 / 77 \\
28 / 3 / 75 \\
20 / 4 / 75 \\
3 / 5 / 75 \\
7 / 4 / 77\end{array}$ & $\begin{array}{c}1 \\
1 \\
2 / 3 \\
2 / 3 \\
2 / 3 \\
1 \\
1 / 3 \\
1 \\
1 \\
2 / 3 \\
1 \\
1 / 6 \\
1 / 3 \\
2 / 3 \\
1\end{array}$ & $\begin{array}{l}2.8 \pm 0.5 \\
2.3 \pm 0.5 \\
2.5 \pm 0.7 \\
\leqslant 2.9 \\
\leqslant 2.1 \\
2.0 \pm 0.5 \\
\leqslant 2.6 \\
2.9 \pm 0.5 \\
3.2 \pm 0.5 \\
\leqslant 2.4 \\
2.1 \pm 0.6 \\
\leqslant 2.0 \\
\leqslant 2.0 \\
\leqslant 1.6 \\
3.5 \pm 0.5\end{array}$ \\
\hline \multicolumn{6}{|c|}{ Borings in Wadi Myah region $\left(19^{\circ} \mathrm{N}, 48^{\circ} \mathrm{E}\right)$} \\
\hline $\begin{array}{r}\text { Ly-1081 } \\
-1079 \\
-1078 \\
-1080 \\
-1113\end{array}$ & $\begin{array}{l}\text { WA } 251 \\
\text { MI } 3 \mathrm{~A} \\
\text { MI } 1 \mathrm{U} \\
\text { MI } 2 \mathrm{~K} \\
\text { MI } 2 \mathrm{WP}\end{array}$ & $\begin{array}{l}\text { Neogene } \\
\text { Alat Kobar } \\
\text { Alat Kobar } \\
\text { Alat Kobar } \\
\text { Wassia }\end{array}$ & $\begin{array}{l}27 / 4 / 75 \\
25 / 4 / 75 \\
25 / 4 / 75 \\
26 / 4 / 75 \\
25 / 5 / 75\end{array}$ & $\begin{array}{l}2 / 3 \\
2 / 3 \\
1 \\
2 / 3 \\
1 / 3\end{array}$ & $\begin{array}{l}53.2 \pm 1.7 \\
\leqslant 2.8 \\
\leqslant 1.5 \\
\leqslant 1.7 \\
\leqslant 2.7\end{array}$ \\
\hline \multicolumn{6}{|c|}{ Borings in $\mathrm{Al}$ Quatif $\left(26^{\circ} 35^{\prime} \mathrm{N}, 50^{\circ} 00^{\prime} \mathrm{E}\right)$} \\
\hline $\begin{array}{r}\text { Ly- } 1094 \\
-1095 \\
-1050 \\
-1082 \\
-1083 \\
-1093 \\
-1074 \\
\\
-1084 \\
-1072 \\
-1085 \\
-1073\end{array}$ & $\begin{array}{l}\text { WA } 1682 \\
\text { WM } 1238 \\
\text { WA } 793 \\
\text { Q } 3 \text { A } \\
\text { Q } 2 \mathrm{~K} \\
\text { WA } 1678 \\
\text { S } 394 \\
\text { Q } 1 \text { U } \\
\text { Abqaiq } 835 \\
\text { BU'AYJ } 802 \\
\text { Berri } 809\end{array}$ & $\begin{array}{l}\text { Neogene } \\
? \\
\text { Alat Kobar } \\
\text { Alat Kobar } \\
\text { Alat Kobar } \\
\text { Alat Kobar } \\
\text { Alat Kobar Um } \\
\text { er Radhuma } \\
\text { Um er Radhuma } \\
\text { Wassia } \\
\text { Wassia } \\
\text { Wassia }\end{array}$ & $\begin{array}{l}14 / 5 / 75 \\
15 / 5 / 75 \\
22 / 4 / 75 \\
28 / 4 / 75 \\
29 / 4 / 75 \\
13 / 5 / 75 \\
11 / 4 / 75 \\
\\
29 / 4 / 75 \\
2 / 4 / 75 \\
2 / 5 / 75 \\
9 / 4 / 75\end{array}$ & $\begin{array}{c}1 / 6 \\
2 / 3 \\
2 / 3 \\
2 / 3 \\
2 / 3 \\
1 / 3 \\
1 / 2 \\
1 \\
1 / 6 \\
1 \\
1 / 2\end{array}$ & $\begin{array}{c}91.6 \pm 4.0 \\
3.7 \pm 0.4 \\
\leqslant 1.0 \\
\leqslant 2.8 \\
\leqslant 1.5 \\
\leqslant 3.2 \\
\leqslant 2.0 \\
\\
\leqslant 1.8 \\
\leqslant 2.0 \\
1.6 \pm 0.4 \\
\leqslant 1.5\end{array}$ \\
\hline \multicolumn{6}{|c|}{ Borings in $\operatorname{Harad}\left(14^{\circ} 15^{\prime} \mathrm{N}, 49^{\circ} 00^{\prime} \mathrm{E}\right)$ and Khurais $\left(24^{\circ} 55^{\prime} \mathrm{N}, 48^{\circ} 05^{\prime} \mathrm{E}\right)$ regions } \\
\hline $\begin{array}{r}\text { Ly-1075 } \\
-1096 \\
-1097 \\
-1160 \\
-1199\end{array}$ & $\begin{array}{l}\text { Khurais } \\
\text { HAP } 5 \\
\text { HAP } 43 \\
\text { HD } 1 \text { W } \\
\text { KH } 1 \text { W }\end{array}$ & $\begin{array}{l}805 \\
\text { Um er Radhuma } \\
\text { Um er Radhuma } \\
\text { Wassia } \\
\text { Wassia }\end{array}$ & $\begin{array}{l}14 / 4 / 75 \\
18 / 5 / 75 \\
18 / 5 / 75 \\
5 / 12 / 75 \\
25 / 1 / 76\end{array}$ & $\begin{array}{l}1 / 2 \\
1 / 4 \\
2 / 3 \\
1 / 2 \\
1 / 2\end{array}$ & 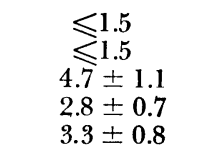 \\
\hline \multicolumn{6}{|c|}{ Vorings in Ain Dar region $\left(25^{\circ} 55^{\prime} \mathrm{N}, 45^{\circ} 10^{\prime} \mathrm{E}\right)$} \\
\hline $\begin{array}{r}\text { Ly-1119 } \\
-1361\end{array}$ & $\begin{array}{l}\text { WA } 603 \\
\text { AD } 804\end{array}$ & $\begin{array}{l}\text { Um er Radhuma } \\
\text { Um er Radhuma }\end{array}$ & $\begin{array}{r}6 / 6 / 75 \\
76\end{array}$ & $\begin{array}{l}1 / 2 \\
1 / 2\end{array}$ & $\begin{array}{l}2.0 \pm 0.3 \\
\leqslant 2.0\end{array}$ \\
\hline \multicolumn{6}{|c|}{ Borings in W Riyad region $\left(24^{\circ} \mathrm{N}, 46^{\circ} 30^{\prime} \mathrm{E}\right)$} \\
\hline $\begin{array}{r}\text { Ly-1076 } \\
-1120\end{array}$ & $\begin{array}{l}\text { WB } 6 \\
\text { N } 5\end{array}$ & $\begin{array}{l}\text { Wassia } \\
\text { Wadi Nisah } \\
\text { Wassia }\end{array}$ & $\begin{array}{c}15 / 4 / 75 \\
9 / 6 / 75\end{array}$ & $\begin{array}{c}1 \\
2 / 3\end{array}$ & $\begin{array}{l}\leqslant 1.1 \\
33.9 \pm 1.3\end{array}$ \\
\hline \multicolumn{6}{|c|}{ Borings in NW Riyad region $\left(27^{\circ} \mathrm{N}, 45^{\circ} 30^{\prime} \mathrm{E}\right)$} \\
\hline $\begin{array}{r}\text { Ly-1115 } \\
-1117 \\
-1114 \\
-1116\end{array}$ & $\begin{array}{l}\text { WA } 1632 \\
\text { S 734 } \\
\text { WA } 1613 \\
\text { WA } 1601\end{array}$ & $\begin{array}{l}\text { Um er Radhuma } \\
\text { Um er Radhuma } \\
\text { Wassia } \\
\text { Wassia }\end{array}$ & $\begin{array}{r}31 / 5 / 75 \\
3 / 6 / 75 \\
30 / 5 / 75 \\
1 / 6 / 75\end{array}$ & $\begin{array}{l}2 / 3 \\
1 / 6 \\
1 / 2 \\
2 / 3\end{array}$ & $\begin{array}{c}110.0 \pm 2.1 \\
\leqslant 4.0 \\
50.7 \pm 1.7 \\
84.7 \pm 2.3\end{array}$ \\
\hline
\end{tabular}

General Comment (BRGM): results prove that five superimposed aquifers are independent from isotopic point of view although there are local phenomena of drainage. 


\section{La Grotte de la Cocalière series, Courry, Gard}

Table 33 lists samples of water from three loci in karstic system of La Cocalière grotto $\left(44^{\circ} 19^{\prime} \mathrm{N}, 3^{\circ} 10^{\prime} \mathrm{E}\right) .501$ samples coll and quickly prepared by strong acidification in lab by $\mathbf{G}$ Marien, $\mathrm{C}$ Pachiaudi, and $\mathbf{P}$ Renault, Geol Dept, Univ Lyon. Measurements were made as part of study of origin and turnover of calcium bicarbonates in karstic systems and on hydrogeol of fissured limestones (Burger, 1980). Samples were taken either from bottom of two underground rivers right tributary (AD) or left tributary (AG) or outflow of system (R). Only undiluted samples were measured in proportional counters; others in liquid scintillation devices.

TABLE 33

La Cocalière Grotto

\begin{tabular}{rlrcrr}
$\begin{array}{c}\text { Sample } \\
\text { no. }\end{array}$ & Loc & Date & $\begin{array}{c}\text { Dilution } \\
\text { ratio }\end{array}$ & $\begin{array}{c}\delta^{13} \mathrm{C} \\
(\% \text { PDB } \pm 0.1)\end{array}$ & $\begin{array}{r}\text { Activity } \\
\text { (\% of modern) }\end{array}$ \\
\hline Ly-1110 & AD & $7 / 5 / 75$ & $1 / 3$ & -14.5 & $97.1 \pm 3.2$ \\
-1111 & R & $27 / 6 / 75$ & $1 / 4$ & -12.6 & $106.6 \pm 2.6$ \\
-1112 & AD & $27 / 6 / 75$ & $1 / 6$ & -15.3 & $96.5 \pm 4.6$ \\
-1129 & R & $2 / 6 / 75$ & $1 / 4$ & -11.5 & $110.3 \pm 5.8$ \\
-1130 & AD & $2 / 10 / 75$ & $1 / 3$ & -7.3 & $123.2 \pm 4.5$ \\
-1131 & R & $27 / 11 / 75$ & $4 / 15$ & -7.3 & $124 \pm 4.4$ \\
-1132 & R & $2 / 12 / 75$ & $1 / 3$ & -7.4 & $119.8 \pm 3.8$ \\
-1174 & AD & $28 / 1 / 76$ & $1 / 5$ & -13.2 & $120.2 \pm 4.9$ \\
-1277 & AG & $11 / 3 / 76$ & 1 & -17.1 & $113.7 \pm 2.5$ \\
-1278 & R & $11 / 3 / 76$ & 1 & -15 & $117.1 \pm 2.5$ \\
-1227 & AG & $26 / 5 / 76$ & $4 / 15$ & -11.0 & $121.4 \pm 4.4$ \\
-1228 & AD & $27 / 5 / 76$ & $1 / 6$ & & $119.9 \pm 6.8$ \\
-1336 & AD & $22 / 7 / 76$ & $1 / 6$ & -14.2 & $102.3 \pm 5.0$ \\
-1337 & AD & $21 / 9 / 76$ & $4 / 15$ & & $116.4 \pm 4.3$ \\
-1338 & R & $21 / 9 / 76$ & $1 / 5$ & -18.3 & $91.1 \pm 4.3$ \\
-1339 & AG & $2 / 12 / 76$ & $1 / 5$ & -14.5 & $112.2 \pm 4.6$ \\
-1340 & AD & $2 / 12 / 76$ & $1 / 6$ & -13.2 & $112.3 \pm 5.3$ \\
-1539 & AG & $10 / 12 / 77$ & $1 / 4$ & & $123.7 \pm 4.2$ \\
-1540 & R & $10 / 12 / 77$ & $2 / 3$ & -15.3 & $116.2 \pm 3.1$ \\
-1541 & AD & $22 / 4 / 77$ & 1 & -13.8 & $107.9 \pm 1.6$ \\
-1542 & AG & $22 / 4 / 77$ & $2 / 3$ & -14.3 & $96.3 \pm 3.2$ \\
-1543 & AG & $30 / 5 / 77$ & 1 & -13.0 & $121.5 \pm 1.9$ \\
-1544 & R & $30 / 5 / 77$ & 1 & -11.6 & $120.7 \pm 2.0$ \\
-1545 & AG & $9 / 6 / 77$ & $1 / 3$ & -9.0 & $122.0 \pm 5.2$ \\
-1546 & R & $9 / 6 / 77$ & 1 & & $119.5 \pm 3.1$ \\
-1547 & R & $17 / 6 / 77$ & 1 & -13.1 & $122.5 \pm 3.1$ \\
-1548 & AG & $17 / 6 / 77$ & $1 / 3$ & -15.1 & $118.8 \pm 4.6$ \\
\hline & & & & &
\end{tabular}

General Comment: results are very homogeneous and confirm reliability of sampling, transport, and preparation procedures. Compared to other climatologic data (mainly precipitation and evapotranspiration), highest activities (ca $120 \%$ modern) correspond to periods with normal precipitation when karstic system mainly let flow 1-yr-old waters. During low-water periods lowest activities (up to $91 \%$ modern) indicate temporarily larger contribution of waters $>1$ yr old, probably stocked in fissures of limestone. On larger scale, same phenomena was previously studied by numerous ${ }^{14} \mathrm{C}$ measurements in Fontaine de Vaucluse system (see Le Chene series, R, 1971, v 13, p 65). 


\section{REFERENCES}

Ambert, P and Tavoso, A, 1981, Les formations quaternaires de la Vallée du Tarn, entre Millau et Saint-Rome de Tarn: Paleobiol continentale, v 12, no. 1, p 185-193.

Archambault, M, 1967, Recensement provisoire des arbres et formations forestiéres dans le bassin de la moyenne Durance alpestre: Acad Sci (Paris) Comptes rendus, ser D, p 2101-2104, 2284-2287.

Archambault-Guézou, J, in press, Apport des analyses paléologiques à l'interprétation historique des cordons littoraux holocènes de la région d'Aigues-Mortes (Gard, France): Rev Géol Méditerranéenne, in press.

Baxter, M S, 1983, Tree ring replicate study, in Mook, W G and Waterbolk, H T, eds, Internatl symposium on C-14 and archaeol, 1st, Proc: Groningen, PACT Jour, in press.

Bazile, F, (ms) 1977, Recherches sur le passage du Würm ancien au Würm récent et sur le début du Würm récent en Languedoc oriental: Thesis, Univ Montpellier, 230 p.

Bazile, F and Bazile-Robert, E, 1981, Position chronostratigraphique du Solutréen à Pointes à cran de la Baume d'Oullins, Le Gorn (Gard) et La Bastide de Virac (Ardèche): Etudes préhist, p 10-15.

Bazile, F, L'Homer, A, Thommeret, J, and Thommeret, Y, 1981, Etapes de l'édification des cordons littoraux d'Aigues-Mortes depuis 7000 BP: Livret Guide Excursion AFEO 1981, p 71-83.

Beaulieu, J L de, 1972, Analyses polliniques des tourbes éemiennes de Saint-Paul les Durance (Bouches du Rhône): Assoc fr Etude Quaternaire, Bull, v 1972, no. 3 p 195-205.

(ms) 1977, Contribution pollenanalytique à l'histoire tardiglaciaire et holocène de la végétation des Alpes méridionales françaises: Thesis, Univ Marseille, 358 p.

Beeching, A, 1979, La question du Cortaillod et des stades culturels qui l'ont précédés: Etudes Prehist, v 13, p 15-17.

(ms) 1980, Introduction à l'étude des stades néolithiques et chalcolithiques dans le bassin du Rhône moyen: Thesis, Univ Lyon, $3 \mathrm{v}$.

Beeching, A and Thomas-Beeching, J, 1975, L'habitat chasséen de La Roberte à Chateau-neuf du Rhône (Drôme): Etudes Prehist, v 12, p 23-32.

Bezinge, A and Vivian, R, 1976, Climat de la période Holocène: La Houille Blanche, v 1976, no. 6-7, p 441-460.

Bonifay, E, 1962, Recherches sur les terrains quaternaires dans la Sud-Est de la France: Bordeaux, Delmas Press, 194 p.

Bonnet, C and Plouin, S, 1979, Nouvelles fouilles dans les terres du Kastenwald, Appenwhir VI: Cahiers alsaciens Archeol Art Hist, v 1979, p 23-28.

Bornand, M and Guyon, A, 1979, Etudes pédologiques dans la Haute Vallée du Rhône, aménagement de Chautagne et de Belley: Doc SES INRA Montpellier, v 460, 92 p.

Bourdelle, Y, 1979, L’Abri Durif à Enval: Actes Colloquium La fin des temps glaciaires en Europe: Paris, CNRS Press, p 523-530.

Bouvier, J M, 1969, Existence de Magdalénien supérieur sans harpon: Preuves stratigraphiques: Acad Sci (Paris) Comptes rendus, ser D, v 268, p 2865-2866.

Bovington, C, Mahdavi, A, and Masoumi, R, 1973, Tehran University Nuclear Centre radiocarbon dates II: Radiocarbon, $v$ 15, p 592-598.

Bricout, J and Koziet, J, 1978, Flavor of foods and beverages: New York, Academic Press, $199 \mathrm{p}$

Burger, A, 1980, Rapport géologique sur le milieu karstique: Soc Geol France, Mém hors ser, $\mathrm{v} 11, \mathrm{p} 29-36$

Burleigh, R, Hewson, A, Meeks, N, Sieveking, G, and Longworth, I, 1979, British Museum natural radiocarbon measurements X: Radiocarbon, $\mathbf{v} 21$, p 41-47.

Burollet, P F, Clairefond, P, and Winnock, E, 1979, La Mer Pélagienne, Geol Méditerranéenne, $\mathrm{v} 6$, no. $1,345 \mathrm{p}$.

Caillaud, $R$ and Lagnel, E, 1967, Sépulture collective de Bardouville (carrière de Beaulieu): Annales Normandie, v 17, no. 4, p 281-315.

Camps, G, Delibrias G, and Thommeret I, 1968, Chronologie absolue et sucession des civilisations préhistoriques dans le Nord de l'Afrique: Lybica, v 16, p 9-28.

Campy, M, 1980, Le lac de Chaillexon: origine, histoire et avenir: Rev Centre Univ Etudes régionales Besançon, v 1980, $180 \mathrm{p}$.

Carité, D, 1977, Quelques observations et déductions sur le Nouakchottien: Club amis Nature en Mauritanie, Bull, v 6, p 18-30.

Chavanne, B, (ms) 1980, Recherches archéologiques sur la moyenne vallée du Sénégal: Thesis, Univ Aix en Provence, 280 p. 
Clot, A, 1982, Le Lynx des Moustayous: Centre Aturien Recherches sous Terre, Bull, $\mathrm{v} 4, \mathrm{p} 1-10$.

Clot, A, Coquerel, R, and Omnès, J, 1978, Une triple inhumation de Bronze ancien à La Gourgue d'Asque (Hautes-Pyrénées): Soc Hist Nat Toulouse, Bull, v 114, p 112-113.

Clot, A and Omnès, J, 1980, Premiers datages radiocarbone du Magdalénien des Hautes Pyrénées: Soc Prehist Fr Bull, v 76, no. 10-12, p 324-339.

Cogoluènhes, A, (ms) 1977, Vers une utilisation des données multiples en anthropologie: Thesis, Univ Lyon 1, $120 \mathrm{p}$.

Colardelle, M, (ms) 1980, Sépulture et traditions funéraires dans les Alpes françaises du Nord: Thesis, Univ Grenoble, $320 \mathrm{p}$.

Coquerel, R, 1966a, Etude des tumulus de Lamarque-Pontacq: OGAM, v 107-108, p $419-432$.

- 1966b, Le mobilier du Tumulus Tugayé: OGAM, v 105-106, p 177-188.

Coursaget, J and Le Run, J, 1966, Gif-sur-Yvette natural radiocarbon measurements I: Radiocarbon, v 8, p 126-141.

Coûteaux, M, 1978, Analyses polliniques à Peyrebeille, Mezillac-et-Mazan (Ardèche), évolution et génèse des sols podzoliques à horizon noir profond: Pollen et Spores, v 20, p 485-495.

1981, Présence, datage et signification phytosociologique de macrorestes de Pinus et de pollen de Pinus cembra $L$ dans le vallon de Lavey, Massif des Ecrins (Isère, France): Actes Colloquium Palynol Paleontol, Genève 1981, p 65-70.

Ccûteaux, M and Evin, J, 1981, Etude palynologique et datage par le radiocarbone de dépôts fluvio-glaciaires aux Deux-Alpes (Isère, France): Acad Sci (Paris) Comptes rendus, ser H, v 292, p 1235-1238.

Crémillieux, A, 1979, Comptes rendus d'activité annuelle en haute vallée de la Loire: Nouv Archives Mus Lyon, v 17 supp, p 37-41.

1980, L'abri sous roche de la Baume d'Arlempdes (Haute-Loire), Préhistoire et Histoire: Cahiers Préhist, v 1980, Paris, CNRS Press, 183 p.

Daniel, J L, Daugas, J P, Debenath, A, and Raynal, J P, 1979, Découverte de restes humains dans un paléo-chenal du ruisseau "La Gensat" à Chapeau-Rouge, commune de Ménétrol (Puy-de-Dôme): Soc Anthropol SO Bull, v 14, no. 1, p. 19-28.

Daugas, J P and Malacher, F, 1978, Les sépultures du Creux-Rouge à Clermont-Ferrand (Puy-de-Dôme), Néolithique et Gallo-Romain: Soc Anthropol SW Bull, v 12, p 2-10.

Daugas, J P and Raynal, J P, 1980, Essai sur la Nèolithisation dans le Sud du Massif Central: Actes Colloquium Néolithique, Sens 1980: Soc archeol Sens Cahiers, v 1, p 85-89.

Debénath, A and Sbihi-Alaoui, F Z, 1979, Découverte de deux nouveaux gisements préhistoriques près de Rabat (Maroc): Soc Préhist Fr Bull, v 76, p 11-14.

Delibrias, $G$ and Evin, J, 1979, Datations par le radiocarbone des sédiments de la Mer Pélagienne: Geo Mediterranéenne, v 6, no. 1, p 285-290.

Delibrias, G, Guillier, M T, and Labeyrie, J, 1970, Gif natural radiocarbon measure. ments V: Radiocarbon, v 12, p 421-443. p 280-320.

1972, Gif natural radiocarbon measurements VII: Radiocarbon, v 14, 197 p 15-94.

Delluc, B and Delluc, G, 1981, La grotte ornée de Comarque à Sireuil, (Dordogne): Gallia-Préhist, v 24, no. 1, p 1-97

Desbrosse, R, 1981, Périgordien et Aurignacien anciens de la Mère Clochette à Rochefort sur-Nénon (Jura), in Hommage au Prof Millotte: Annales litt, Univ Besançon, $\mathrm{v} 1981$.

Deshayes, J, 1976, Turneg Tepe: Iran, v 15, p 169-171.

Einsele, P, Elouard, P, Hern, D, Köghr, F G, and Scwartz, H U, 1977, Source and biofacies of late Quaternary sediments in relation to sea on the shelf off Mauritania, West Africa: Meteor Forschungsergeb, v 26, p 1-43.

Escalon de Fonton, M, 1976a, Les civilisations de l'Epipaléolithique et du Mésolithique en Languedoc Oriental: La Préhistoire française: Paris, CNRS Press, v 1, no. 2, p 1382-1389.

1976b, Informations archéologiques: Provence-Côte d'Azur: Gallia préhist, v 19, no. 2, p 581-606.

Evin, J, 1983, La datation avec les matériaux d'origine terrestre (Dating with terrestrial materials), in Mook, W G and Waterbolk, H T, eds, Internatl symposium on C-14 and archaeol, 1st, Proc: Groningen, PACT Jour, in press. 
Evin, J, Gilet, N. Legigan, P, and Thibault, C, 1979, Essai de datation absolue d'un horizon humifère inclus dans les sables éoliens des Landes: Comptes rendus, Cong Soc savantes, 104th, Vol Sci III, p 63-72.

Evin, J, Longin, R, Marien, G, and Pachiaudi, C, 1971, Lyon natural radiocarbon measurements II: Radiocarbon, v 13, p 52-73.

Evin, J, Longin, R, and Pachiaudi, C, 1969, Lyon natural radiocarbon measurements I: Radiocarbon, v 11, p 112-117.

Evin, J, Maréchal, J, Pachiaudi, C, and Puisségur, J J, 1980, Conditions involved in dating terrestrial shells, in Stuiver, $M$ and $\mathrm{Kra}, \mathrm{R}$, eds, Internatl ${ }^{14} \mathrm{C}$ conf, 10th, Proc: Radiocarbon, v 22, no. 2, p 545-555.

Evin, J, Marien, G, and Pachiaudi, C, 1973, Lyon natural radiocarbon measurements III: Radiocarbon, v 15, p 134-155.

1973, Lyon natural radiocarbon measurements IV: Radiocarbon, v 15, p 514533.

1975, Lyon natural radiocarbon measurements V: Radiocarbon, v 17, p 4-34. 1976, Lyon natural radiocarbon measurements VI: Radiocarbon, $v$ 18, p 6088.

57.

1978, Lyon natural radiocarbon measurements VII: Radiocarbon, v 20, p 19. p $405-452$.

Fagès, $G$ and Chauviré-Mourer, $C$, in press, La flûte en os d'oiseau de la grotte sépulcrale de Veyreau et inventaire des flutes préhistoriques d'Europe: Soc préhist fr, Mem, in press.

Farizier, M, (ms) 1980, Recherches sur les macroflores des tufs quaternaires du Sud de la France: Thesis, Univ Montpellier, p 266.

Faure, $\mathbf{H}$ and Elouard, P, 1967, Schéma des variations du niveau de l'Océan Atlantique sur la côte Ouest de l'Afrique depuis 40,000 ans: Acad sci (Paris) Comptes rendus, ser $\mathrm{D}, \mathrm{v}$ 265, p 784-787.

Faure, H, Fontes, J C, Hébrard, L, Monteillet, J, and Pirazzoli, P A, 1980, Geoidal change and shore-level tilt along Holocene estuaries: Senegal river area, West Africa: Science, v 210, p 421-423.

Forsyth-Major, C, 1930, Resti di un Orso trovato in una grotta vicino a Monte Estremo (Filosorma, Corsica): Archivio Stor Corsica, p 367-370.

Gilet-Blain, N, Marien, G, and Evin, J, 1980, Unreliability of ${ }^{14} \mathrm{C}$ dates from organic matter of soils, in Stuiver, $\mathrm{M}$ and $\mathrm{Kra}, \mathrm{R}$, eds, Internatl ${ }^{14} \mathrm{C}$ conf, 10th, Proc: Radiocarbon, v 22, no. 3, p 919-929.

Gilles, R, 1975, L'habitat du Néolithique final des Bruyères (basse vallée de l'Ardèche): Etudes préhist, v 12, p 1-13.

Gilot, E, 1969, Louvain natural radiocarbon measurements VII: Radiocarbon, v 11, p 106-111. p 1970, Louvain natural radiocarbon measurements IX: Radiocarbon, $v 12$,

Girard, J, 1980, Les troglodytes de la Proto-historie sénégalaise: Le Soleil, v 20-6-80, p 4.

Jarrige, J F and Lechevallier, M, 1980, Les fouilles de Mehrgarh (Pakistan), Problèmes chronologiques: Paléorient, v 6, p 253-258.

Kozłowski, J K, 1962, Stanowisko przemyslu magdalenskiego jaskini Maszyckiej; le site magdalénien dans la grotte de Maszycka: Maderialy Arceol, v 4, p 1-10.

Koukouli-Chrysanthaki, C, 1980, Oekismos tis hysteris epochis chalkou ston Aggistas Serron: Anthropol, v 1, p 54-85.

Lagier-Bruno, L, 1981, Le parc à moutons et la cabane de berger de Santourin, commune de Billième, Savoie: Le Bugey, v 1981, no. 3, p 1-19.

Laurent, R, 1968, Note préliminaire sur la pirogue monoxyle du Lac de Paladru: Rhodania, v 1968, p 63-68.

Lautier, J, in press, La grotte de La Pyramide (Penne, Tarn): Actes Cong Soc préhist fr, $v 21$, in press.

Lepage, L, 1980, La camp de la Vergentiéres à Cohons (Haute-Marne), rapport avec les régions avoisinantes: Prehist Protohist en Champagne-Ardenne, v 1980, p 139-165.

Lenoir, M, 1977, Les industries du Paleolithique supérieur terminal des basses vallées de la Dordogne et de la Garonne: Actes Colloquium, La fin des temps glaciaires en Europe, v 1, p 401-423.

Leroi-Gourhan, A, 1973, Le paysage au temps des graveurs de la Grotte de "La Marche": Barcelona, Univ Barcelona Press, p 101-108. 
Le Tensorer, J M, (ms) 1980, Recherches sur le Quaternaire en Lot-et-Garonne, stratigraphie, paléoclimatologie et préhistoire paléolithique: Thesis, Univ Bordeaux, p 365-367.

1981, Le Paléolithique de l'Agenais: Cahiers Quaternaire, v 3, 526 p.

Lévèque, $F$ and Vandermeersch, B, 1980, Découverte de restes humains dans un niveau castelperronien à Saint-Cézaire (Charente-Maritime): Acad Sci (Paris) Comptes rendus, ser $\mathrm{D}, \mathrm{v} 291, \mathrm{p} 187$.

Libby, W JF, 1952, Radiocarbon dating: Chicago, Univ Chicago Press, viii, 124 p.

Lintz, G, 1977, Les canalisations gallo-romaines en bois: Soc Lettres Sci Arts Corrèze, Bull, v 80, p 43-66.

Livache, L, 1976, Les civilisations de l'Epipaléolithique et du Mésolithique en HauteProvence et en Vaucluse: La Préhist fr: Paris, CNRS press, v 1, no. 2, p 1379-1381.

Longin, R, 1971, New method of collagen extraction for radiocarbon dating: Nature, v 230, p 241-242.

Mazière, G, 1980, Information archéologique de la circonscription Limousin: Gallia préhist, v 23, no. 2 , p 362-365.

Mordant, $\mathrm{C}$ and Mordant, D, 1977, Les Bois des Refuges à Misy-sur-Yonne, Seine-etMarne: Soc Prehist fr, Bull, v 74, no. 1, p 420-462.

Mordant, C and Poitout, B, 1980, Le Néolithique moyen récent dans le Bassin de 1'Yonne: Actes Colloquium Néolithique Sens: Soc archéol Sens, Cahiers, v l, p $171-178$.

Mordant, D, 1967, Le Néolithique du Gros-Bois à Balloy (Seine-et-Marne): Soc Préhist fr, Bull, v 64, no. 2, p 348-366.

Morelon, S, 1974, La gisement préhistorique de La Touvière, commune d'Arbignieu: Doc Lab Géol Fac sci Lyon, v 56.

Nébois, R, (ms) 1974, Plateaux et chaines de la Lucanie Orientale et des Pouilles, Etudes de morphologie: Thesis, Univ Paris IV.

Omnès, J, 1981, Inventaire préhistorique de la commune de Lourdes (Hautes-Pyrénées): Lavedan et Pays Toy, v 13, p 67-86.

Philibert, D and Debard, E, 1977-78, La grotte de La Pécoulette à Lagorce (Andèche): Etudes Prehist, v 14, p 7-12.

Philippe, M, Mourer-Chauviré, C, and Evin, J, 1981, Les gisements paléontologiques quaternaires des Causses de Martel et de Gramat (Corrèze et Lot); faune et chronologie: Nouv Archives Mus Hist Nat Lyon, v 18 supp, p 57-67.

Piette, J, 1973-74, Le site néolithique des Grèves de Frécul à Babuise-Courtevent (Aube): Groupe Recherche archéol Nogentais, Bull, v 10, p 3-18.

Poplin, F, 1980, Syviornis neocaledonia $\mathrm{n} \mathrm{g}, \mathrm{n}$ sp (Aves), ratite èteint de la NouvelleCalédonie: Acad Sci (Paris) Comptes rendus, ser D, v 290, p 691-694.

Pradel, L, 1950, Le Solutréen supérieur de la Grotte de La Tannerie, commune de Lussacles Châteaux (Vienne): Soc Prehist fr, Bull, v 1950, p 467-471.

1958 La grotte Magdalénienne de La Marche, commune de Lussac-les Châteaux (Vienne): Soc Préhist fr, Mém, v 5, p 170-191.

1979, L'abri périgordien de Laraux, commune de Lussac les Châteaux (Vienne): Nouvelles constatations et datations par le ${ }^{14} \mathrm{C}$ : Anthropol, v 83, p 439-454.

Raynal, J P and Daugas, J P, 1979, Etudes quaternaire en Grand Limagne d'Auvergne, II: les dépots de versant du Creux-Rouge, commune de Clermont-Ferrand (Puyde-Dôme): Nouv Archives Mus Hist Nat Lyon, v 17 supp, p 87-95,

1981, Arguments en faveur d'un age rissien pour la fossile humain découvert en 1876 au lieu-dit Les Riveaux à Espaly-Saint-Marcel, (Haute-Loire): Acad sci (Paris) Comptes rendus, ser D, v 292, p 1501-1504.

Reille, M and Pons, A, 1982, L'histoire récente de Pinus-silvestris $L$ en Margeride, (Massif Central, France), et la sígnification de cette essence dans la végétation actuelle: Acad Sci (Paris) Comptes rendus, ser 3, p 471-474.

Richard, G, 1980, Le Dolmen de la Pierre Godon à Soignoles, Commune de Tillay le Peneux (Eure-et-Loir): Actes Cong St Amand, 1979: Mus St Vic, Bull, v 1980, p 148-157.

Richard, $G$ and Vintrou, J, 1979, Les sépultures sous dalle des Maraules et de La Chaise à Malesherbes (Loiret) et le problème de leur appartenance culturelle: Actes Colloque Néolithique Châlons sur Marne: Préhist et Protohist ChampagneArdennes, v 175-181.

Sato, J, Sato, T, and Suzuki, H, 1968, University of Tokyo radiocarbon measurements I. Radiocarbon, v 10 p 144-148.

Séronie-Vivien, M R, 1972, L'industrie lithique de la grotte de La Bergerie des QuatreChemins à Caniac, Lot: Soc Linnéenne Bordeaux, Bull, v 2, no. 3, p 65-71. 
Séronie-Vivien, M R, in press, La grotte de Pégourié à Caniac du Causse (Lot); le gisement Azilien et Magdalénien initial: Actes Cong Soc Préhist Fr, Montauban-Cahors, in press.

in press, La grotte de La bergerie des Quatre Chemins à Caniac du Causse (Lot) gisement Magdalénien et du Bronze Ancien/Moyen: Actes Cong Soc Préhist Fr, Montauban-Cahors, in press.

Svoboda, J, 1979, Stratigraphy in the Mesolithic settlement in the rock-shelter at Hermansky Polomene Mts, North Bobemia: Anthropol Brno, v 17, p 87-93.

Thévenot, J P, 1978, Information archéologique de la circonscription Bourgogne; le Camp de Chassey: Gallia-Préhist, v 21, p 585-588.

Thilmans, G and Ravisé, A, in press, Protohistoire du Sénégal; Les sites du Fleuve: Mem IFAN, v 91, in press.

Thinon, M, 1978, La pédoanthrapologie: Nouvelle méthode d'analyse phytochronologique depuis le Néolithique: Acad Sci (Paris) Comptes rendus, ser D, v 287, p 1203-1206.

Triat-Laval, H, 1981, Analyse pollinique d'une séquence tourbeuse de Provence Orientale (Seillons-Source d'Argens, Var, France): Ecol mediterranea, v 7, no. 2, p 50-60.

Utrilla-Miranda, P, 1980, Fechas de C 14 para la prehistoria del Valle del Ebro: CaesarAugusta, v 51-52, p 5-10. in press, El yacimiento de la cueva de Abauntz (Arrais, Navarra): Trabajos Argueol Navarra, v 3, in press.

Valastro, S, Davis, E M, and Varela, A, 1975, University of Texas, Austin, radiocarbon dates X: Radiocarbon, v 17, p 52-98.

Vertet, H, 1981, Sauvetage d'un bateau fluvial antique dans la Loire: Archéol, v 150, p 74 .

Vivian, R, 1975, Les glaciers des Alpes occidentales: Grenoble, Allier Press, 514 p.

Vogel, J C and Waterbolk, H T, 1965, Groningen radiocarbon dates IV: Radiocarbon, v 5, p 163-202.

1967, Groningen radiocarbon dates VII: Radiocarbon, v 9, p 107-155.

Wolf, J J, 1978, La contribution de l'archéologie à l'histoire de Sierentz et de sa région: Annuaire Soc Hist sundgauvienne, v 1978, p 140-160. 